\title{
INVESTIGATION OF A DIRECTION FINDING ANTENNA ARRAY FOR RADIO FREQUENCY IDENTIFICATION
}

by

\author{
Garret McKerricher \\ A thesis submitted to the Department of Electronics \\ in conformity with the requirements for \\ the degree of Masters of Applied Science
}

\author{
Carleton University \\ Ottawa, Ontario, Canada
}

(September, 2010)

Copyright $\mathbb{C}$ Garret McKerricher, 2010 
Library and Archives

Canada

Published Heritage Branch

395 Wellington Street Ottawa ON K1A ON4 Canada
Bibliotheque et

Archives Canada

Direction du

Patrimoine de l'édition

395, rue Wellington

Ottawa ON K1A ON4

Canada
Your file Votre référence

ISBN: 978-0-494-71520-8

Our file Notre référence

ISBN: 978-0-494-71520-8
NOTICE:

The author has granted a nonexclusive license allowing Library and Archives Canada to reproduce, publish, archive, preserve, conserve, communicate to the public by telecommunication or on the Internet, loan, distribute and sell theses worldwide, for commercial or noncommercial purposes, in microform, paper, electronic and/or any other formats.

The author retains copyright ownership and moral rights in this thesis. Neither the thesis nor substantial extracts from it may be printed or otherwise reproduced without the author's permission.
AVIS:

L'auteur a accordé une licence non exclusive permettant à la Bibliothèque et Archives Canada de reproduire, publier, archiver, sauvegarder, conserver, transmettre au public par télécommunication ou par l'Internet, prêter, distribuer et vendre des thèses partout dans le monde, à des fins commerciales ou autres, sur support microforme, papier, électronique et/ou autres formats.

L'auteur conserve la propriété du droit d'auteur et des droits moraux qui protège cette thèse. $\mathrm{Ni}$ la thèse ni des extraits substantiels de celle-ci ne doivent être imprimés ou autrement reproduits sans son autorisation.
In compliance with the Canadian Privacy Act some supporting forms may have been removed from this thesis.

While these forms may be included in the document page count, their removal does not represent any loss of content from the thesis.
Conformément à la loi canadienne sur la protection de la vie privée, quelques formulaires secondaires ont été enlevés de cette thèse.

Bien que ces formulaires aient inclus dans la pagination, il n'y aura aucun contenu manquant. 


\begin{abstract}
Recent advances in radio frequency identification technology have enabled low cost passive RFID tags to be read at increasingly distant ranges. This has sparked interest in real time location systems using RFID tags. A well known military direction finding technique has been considered a viable solution for locating RFID tags. This technique works on the principle of comparing the phase of the received signal on two or more antennas. A phase comparison antenna array is commonly referred to as an interferometer array. The UHF RFID interferometer places unique size and performance requirements on the antenna elements. Both the microstrip patch and the quadrifilar helix antenna are considered in this work. While microstrip patch antennas are traditionally used in RFID, it is necessary to reduce the size of the antenna for use in the interferometer configuration. The quadrifilar helix is not commercially used for RFID; however the quadrifilar possesses many characteristics that make it an ideal candidate for the application. Most notably the antenna can be designed with a shaped radiation pattern that has the potential to improve the performance of RFID systems. The design, fabrication and testing of these antennas are presented in this work. Arrays have been built and tested using both the microstrip patch and quadrifilar prototypes proving that the interferometer array is a practical solution for finding UHF RFID tags.
\end{abstract}




\section{Acknowledgements}

I would like to acknowledge the encouragement, expertise, and financial support that I have received from many individuals. Dr. Jim Wight, Chancellor's Professor at Carleton University, who provided me with the opportunity to study under his guidance. Jim, I had a great experience throughout my studies, thank you for your generosity and all your great advice. Sanjay Chadha, CEO of S5 Systems, has been enthusiastic about the project and provided financial support and resources. Sanjay, thank you for the opportunity to work with you, the technical and nontechnical experience you provided is greatly appreciated. Aldo Petosa, project leader of antenna design and development at the Communications Research Center in Ottawa, who introduced me to antenna design and provided me with access to resources for accurate measurement of my prototypes. Nagui Mikail, Hardware Manager at Carleton University, who really got me started, made sure I had the necessary resources, and gave me hands on expertise in fabrication. David and Stephen Tilston, owners of Phoenix Antennas, have provided me with valuable advice in antenna design and manufacturing. Matt Hills, RF Hardware Design Specialist of Aeronautical Antennas at Esterline CMC Electronics, helped with the conceptual design stages of the quadrifilar antenna. Gyles Panther, CEO of Tallysman Wireless, for his expertise in antenna design and manufacturing. Ying Shao, DSP Engineer at S5 Systems, who worked in parallel on the direction finding solution, specifically, on the signal processing side of the system. Finally I would like to thank my parents and family for supporting me no matter what path I decided to travel down. 


\section{Table of Contents}

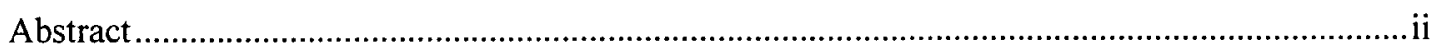



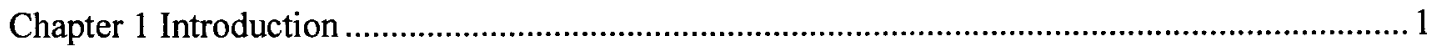

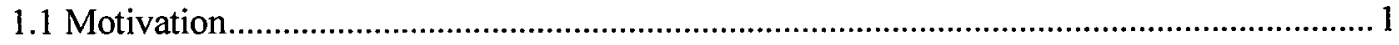

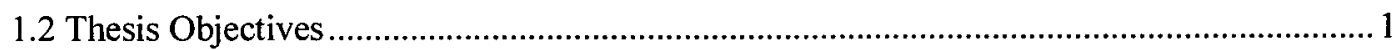

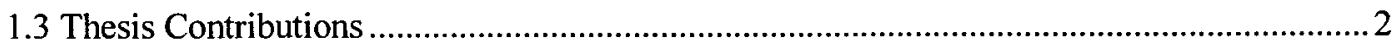

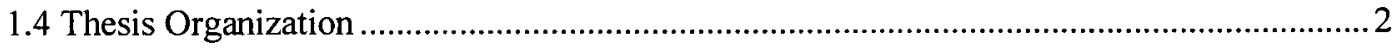

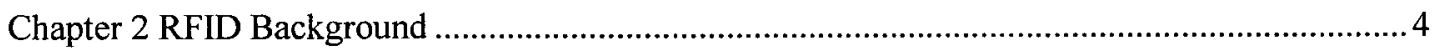

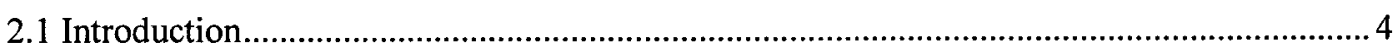

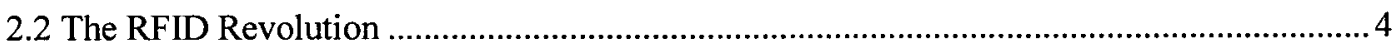

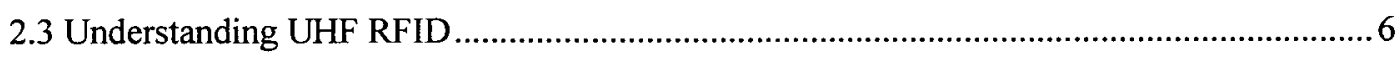

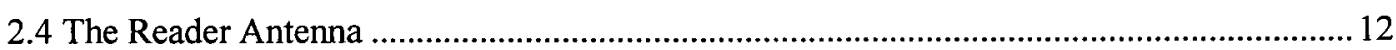

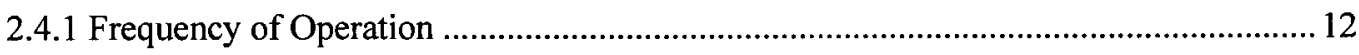

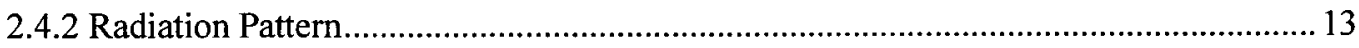

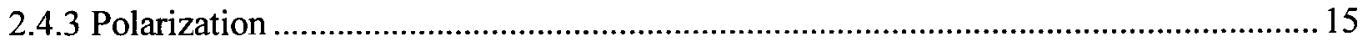

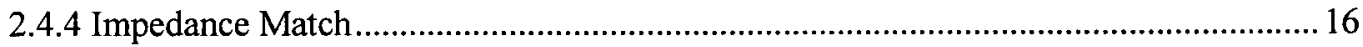

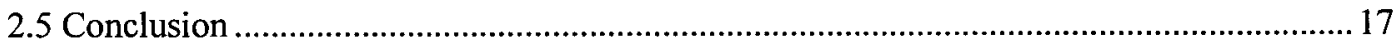

Chapter 3 Direction of Arrival using Phase Comparison ........................................................ 18

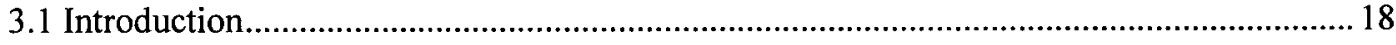

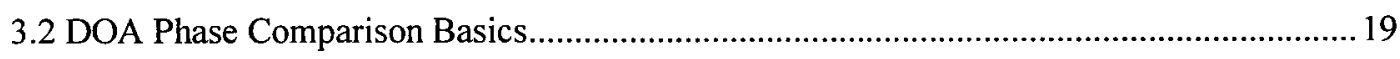

3.2.1 Three Element Interferometer Array ..................................................................... 24

3.2.2 Mathematics of a Three Element Equilateral Triangle Interferometer .........................25

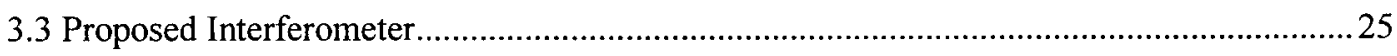

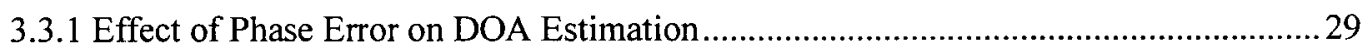

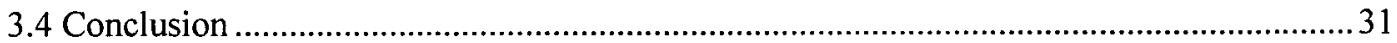

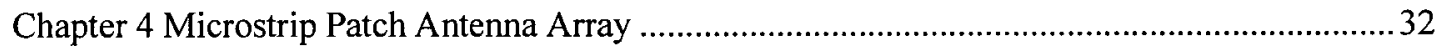

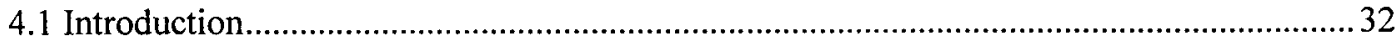

4.2 Circular Patch Antenna Theory - Cavity Model ................................................................... 33

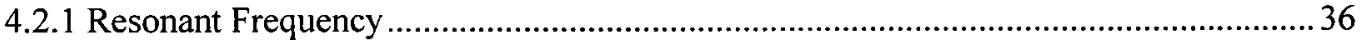






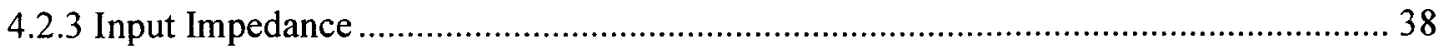

4.2.4 Circular Polarization .......................................................................................... 40

4.3 Microstrip Patch Antenna Array Design ........................................................................ 41

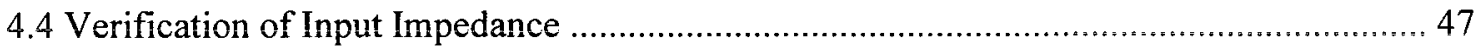

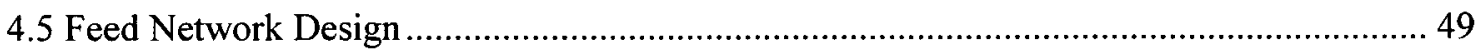

4.6 Microstrip Patch Antenna Fabrication and Testing ……………................................... 51

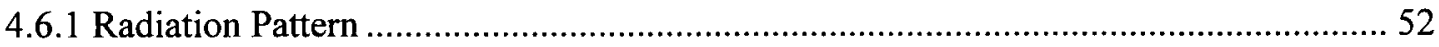

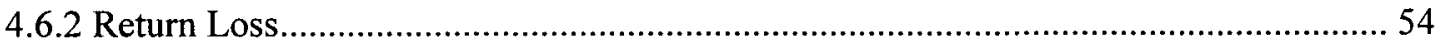

4.6.3 Phase and Gain versus Azimuth Angle ……………………………………….... 55

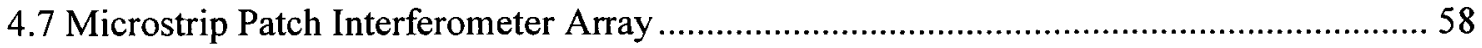

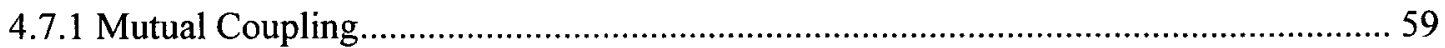

4.7.2 Patch Antenna Array Phase and Gain versus Azimuth Angle................................... 60

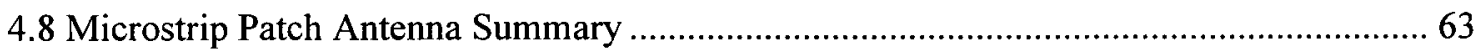

Chapter 5 Quadrifilar Helix Antenna Array ………………………………………………...... 66

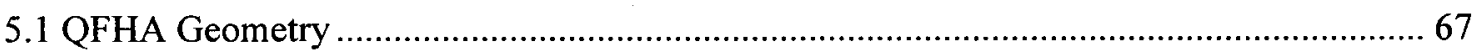

5.2 Understanding the Ideal Radiation Pattern Shape …………………………………..... 70

5.3 The QFHA Radiation Pattern Theory..................................................................... 72

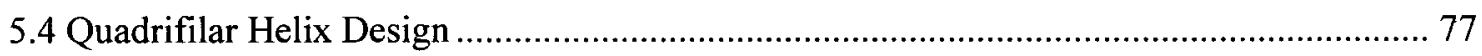

5.4.1 Shaping the Radiation Pattern ................................................................................ 78

5.5 Verification of the HFSS Model............................................................................ 80

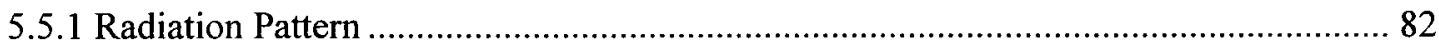

5.5.2 Input Impedance ............................................................................................. 84

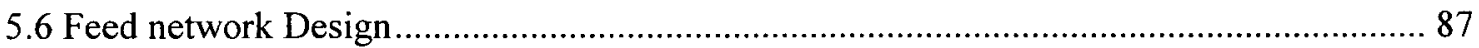

5.7 QFHA Fabrication and Testing ………................................................................. 91

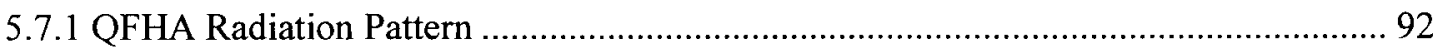

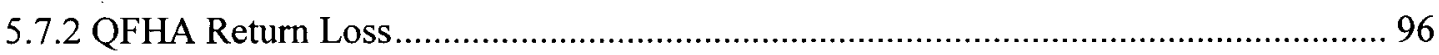

5.7.3 QFHA Phase and Gain versus Azimuth Angle ...................................................... 97

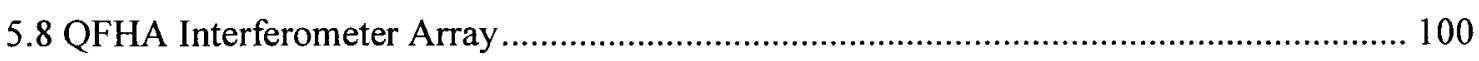

5.8.1 Mutual Coupling ............................................................................................... 101

5.8.2 QFHA Array Phase and Gain versus Azimuth Angle ............................................. 102

5.9 QFHA Summary ................................................................................................ 106

Chapter 6 Conclusions and Future Work ………………................................................ 109 
6.1 Summary and Contributions

6.2 Future Work

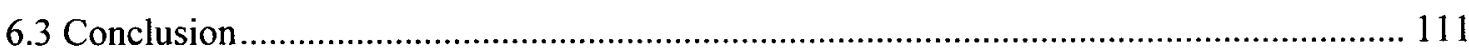

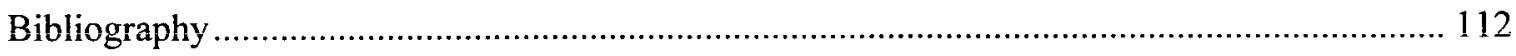




\section{List of Figures}

Figure 2.1: Alien Passive RFID Tags (Left) Antenna (Center) and Reader (Right) [5] ................ 5

Figure 2.2: Matrics Passive RFID Tags (Bottom Left) Reader (Top Left) Antenna (Right).......... 5

Figure 2.3: Use of Backscatter Radiation to Communicate with a Radar Operator [1] ................. 7

Figure 2.4: The use of Backscattered Radiation in Passive RFID ................................................

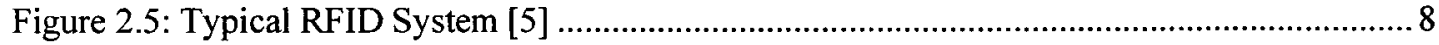

Figure 2.6: HDX RFID Communication Protocol ................................................................... 9

Figure 2.7: Examples of Typical RFID Modulation [2] …....................................................

Figure 2.8: Simple Passive RFID Tag[1] ................................................................................... 10

Figure 2.9: Simplified Physics of Backscatter Signaling [1] ................................................... 11

Figure 2.10: Patch Antenna, with Plastic Radome (Left) and without Radome (Right) [1] ......... 12

Figure 2.11: Antenna Elevation and Azimuth Angles ............................................................. 14

Figure 2.12: Simple Dipole Polarization Example [1] ......................................................... 15

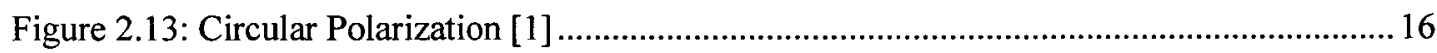

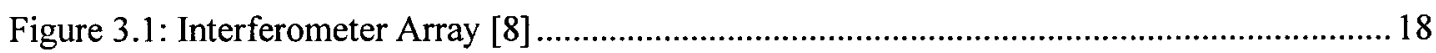



Figure 3.3: Mirror Image Ambiguity for a Two Element Interferometer ....................................21

Figure 3.4: Phase Difference as a Function of AOA for a Two Element Interferometer .............. 22

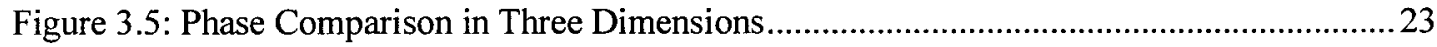

Figure 3.6: Elevation Angle and Effective Separation of the Antennas .......................................24

Figure 3.7: Three Element Interferometer in an Equilateral Triangle Configuration ....................26

Figure 4.1: Impinj Far Field RFID IPJ-A1000 Antenna - Radome (Left) Radome Removed

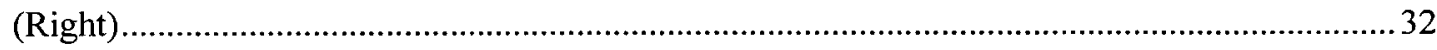

Figure 4.2: Convergence Systems Ltd. UHF RFID Patch Antenna - Radome (Left) Radome

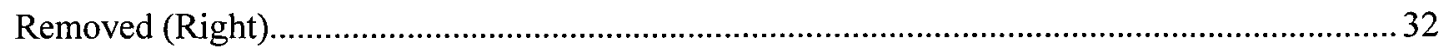

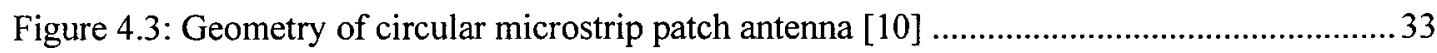

Figure 4.4:Cavity Model and Equivalent Magnetic Current Density for Circular Microstrip Patch



Figure 4.5: Electric and Magnetic Field Pattern of a Circular Microstrip Antenna at Resonance in

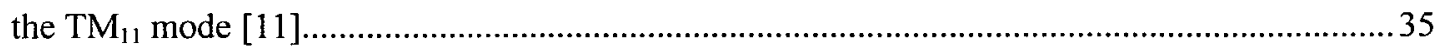


Figure 4.6: Measured and Computed (based on moment method and cavity models) E- and Hplane patterns of circular microstrip patch antenna [10] ............................................................ 38

Figure 4.7: Probe Feed for Microstrip Patch Antenna [10] 39

Figure 4.8: Typical Variation of Resistance and Reactance of Rectangular Microstrip Antenna versus Frequency[10]. 40

Figure 4.9: Circular Polarization Techniques a) Dual Coaxial Feed [10], b) Single Feed with slots[1], c) Single Feed with Elliptical element and tabs[10]. 41

Figure 4.10: Interferometer Antenna Array Configuration........................................................... 42

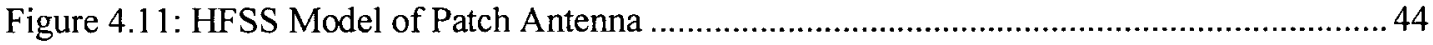

Figure 4.12: Patch Antenna Input Resistance and Reactance as a Function of Coaxial Probe Feed

Offset from Center ( Microstrip Patch HFSS Simulation at $915 \mathrm{MHz}$ ) ..................................... 45

Figure 4.13: Patch Antenna Input Resistance and Reactance as a Function Frequency (HFSS

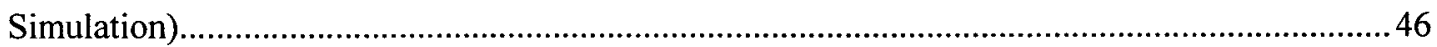

Figure 4.14: Simulated Return Loss (Microstrip Patch HFSS Simulation) .................................. 47

Figure 4.15: Patch Antenna Prototype for Verification of Input Impedance ................................48

Figure 4.16: Comparison of S11 Measurement and Simulation ................................................... 48

Figure 4.17: Comparison of S12 Measurement and Simulation ......................................................49

Figure 4.18: PCB Feed network, Model (Left) Fabricated (Right)................................................50

Figure 4.19: PCB Feed network Measured Phase versus Frequency ...........................................50

Figure 4.20: PCB Feed network Measured Amplitude of Output Signals....................................51

Figure 4.21: Patch Antenna with Feed Network, Top View (Left) Bottom View (Right) ............52

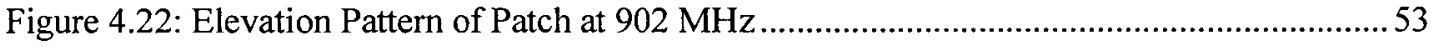

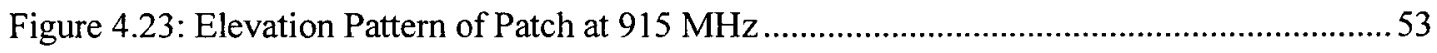

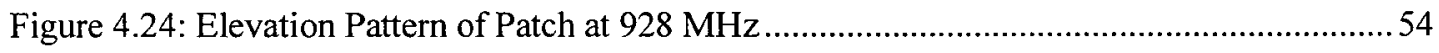

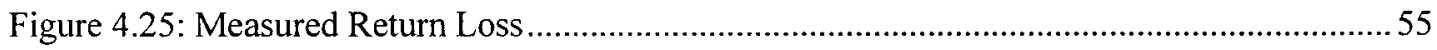

Figure 4.26: Phase versus Azimuth Angle Test Setup at $15^{\circ}$ Elevation Cut ................................56

Figure 4.27: Patch Antenna Phase versus Azimuth Angle at $60^{\circ}$ Elevation Cut .........................56

Figure 4.28: Gain versus Azimuth Angle at a $60^{\circ}$ Elevation Cut ...............................................57

Figure 4.29: Fabricated Patch Antenna Interferometer Array .......................................................5

Figure 4.30: Mutual Coupling between Two Patch Antennas in the Interferometer Configuration

Figure 4.31: Patch Antenna Array versus Azimuth Angle Test Setup at $15^{\circ}$ Elevation Cut .........61 viii 
Figure 4.32: Gain Comparison of Single Patch and Patch in an Array Configuration versus Azimuth Angle ( $915 \mathrm{MHz}$ at $60^{\circ}$ Elevation Angle)

Figure 4.33: Phase Comparison of Single Patch and the Patch in an Array Configuration versus

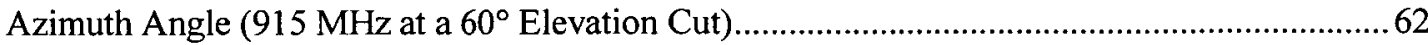

Figure 4.34: UHF RFID Patch Antenna Radiation Pattern (Elevation Cut at $915 \mathrm{MHz}$ ). 64

Figure 5.1: Surrey Satellite Technology - S-Band Quadrifilar Helix Antenna (Left) Elevation

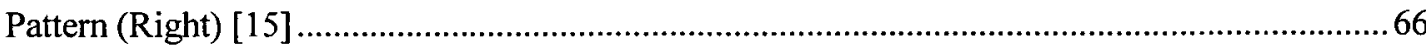

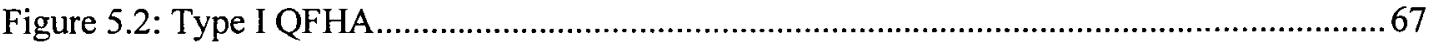

Figure 5.3: QFHA, RHCP Clockwise Windings (a) LHCP Counter-Clockwise Windings (b).... 68

Figure 5.4: Helical Antenna Parameters [10].

Figure 5.5: RFID Application where the Reader Antenna is Centered Above the Coverage Area70 Figure 5.6: Required Gain versus Elevation Angles for the Situation Depicted in Figure 5.5 ...... 72 Figure 5.7: Monofilar Helix, Normal Mode (a) Endfire (Axial) Mode (b) [10] 73 Figure 5.8: Helix Chart Showing the Location of Different Modes of Operation as a Function of the Helix Dimensions (Diameter, Spacing, and Pitch Angle). [12] ............................................. 74

Figure 5.9: Region of Shaped Conical Beam Performance [16] ............................................. 75

Figure 5.10: Elevation Cut of a Shaped Radiation Pattern [16] ................................................ 76

Figure 5.11: Radiation Pattern Characteristics for Quadrifilar with $\mathrm{N}=2$ [16] .............................. 77

Figure 5.12: Radiation Pattern Characteristics with a Teflon Rod for $\mathrm{N}=2 \ldots \ldots \ldots \ldots \ldots \ldots \ldots \ldots \ldots . . . .79$

Figure 5.13: QFHA, HFSS Model (Left) Fabricated Antenna (Right) .......................................... 80

Figure 5.14: Plastic Sheet with Copper Tape ............................................................................ 81

Figure 5.15: Antenna Elevation Pattern at $915 \mathrm{MHz}$, HFSS Ideal Simulation and Measurement 83

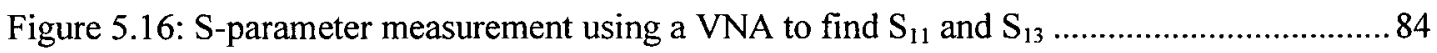

Figure 5.17: Comparison of S11 Simulation (Solid) Measured (Dashed) ....................................85

Figure 5.18: Comparison of S12 Simulation (Solid) Measured (Dashed) ................................... 85

Figure 5.19: Comparison of S13 Simulation (Solid) Measured (Dashed) .....................................86

Figure 5.20: Input Impedance of the HFSS Model Compared with the Fabricated QFHA ...........87

Figure 5.21: QFHA Feed network, HFSS Model (Left) PCB (Right) ........................................... 88

Figure 5.22: PCB Feed network Measured Phase vs. Frequency ................................................. 89

Figure 5.23: Amplitude of Signals Output from Feed network ...................................................90

Figure 5.24: QFHA Prototype: HFSS Model (Left) Fabricated (Right) ......................................92

Figure 5.25: Simulated and Measured (902 MHz) Elevation Pattern of QFHA, $902 \mathrm{MHz}$........... 93 
Figure 5.26: Simulated and Measured (915 MHz) Elevation Pattern of QFHA, $915 \mathrm{MHz} \ldots \ldots \ldots . . .94$

Figure 5.27: Simulated and Measured ( $928 \mathrm{MHz}$ ) Elevation Pattern of QFHA ............................ 94

Figure 5.28: Return Loss of QFHA Simulation Prototype ....................................................... 96

Figure 5.29: The Phase versus Angle Test Setup at a $15^{\circ}$ Elevation Cut ....................................97

Figure 5.30: QFHA Phase Versus Azimuth Angle at 60 degree Elevation Cut............................. 98

Figure 5.31: QFHA Magnitude Versus Azimuth Angle at 60 Degree Elevation Cut................... 99

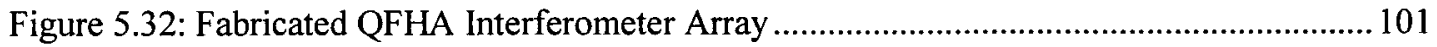

Figure 5.33: Measured Mutual Coupling between Two QFH Antennas in the Interferometer

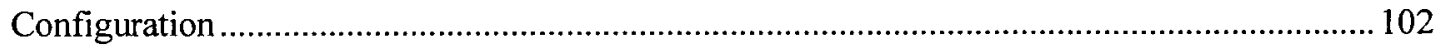

Figure 5.34: QFHA Array Phase versus Angle Test Setup at a $15^{\circ}$ Elevation Cut ..................... 103

Figure 5.35: QFHA Array Mounted in the Anechoic Chamber at the Communication Research

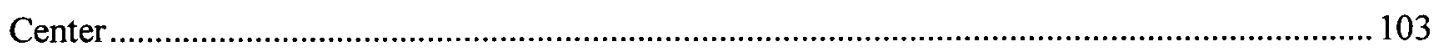

Figure 5.36: Gain Comparison of QFHA and the QFHA in an Array at a $60^{\circ}$ Elevation ........... 104

Figure 5.37: Phase Comparison of QFHA and the QFHA in an Array at a $60^{\circ}$ Elevation .......... 105

Figure 5.38: QFH RFID Reader Antenna Elevation Cut in dBic (Measured at $915 \mathrm{MHz}$ )......... 107 


\section{List of Tables}

Table 3.1: Effect of Phase Error and Elevation Angle on DOA Estimation Accuracy (Monte Carlo Simulation of Proposed Three Element Interferometer) ........................................................... 30

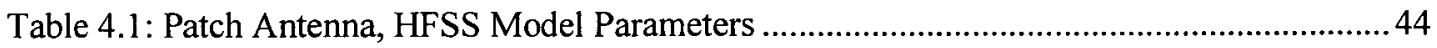

Table 4.2: Azimuth Gain and Phase Deviation of Single Patch Antenna .......................................58

Table 4.3: Patch Array, Gain and Phase Deviation versus Azimuth Angle ..................................63

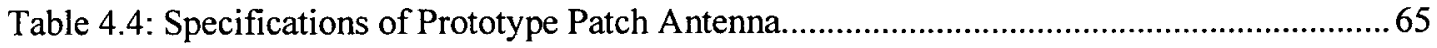

Table 5.1: Parameters of the QFHA Antenna Fabricated and HFSS Model...................................82

Table 5.2: Measured Phase Difference between PCB Feed network Ports at $915 \mathrm{MHz}$..............89

Table 5.3: Return and Mismatch Loss $(50 \Omega)$............................................................................ 91

Table 5.4: Breakdown of Losses in QFHA Simulation at $915 \mathrm{MHz}$.........................................95

Table 5.5: Gain and Phase Deviation of a Single QFHA...................................................... 100

Table 5.6: Gain and Phase Deviation of QFHA Array .......................................................... 105

Table 5.7: QFH RFID Reader Antenna Specifications ........................................................... 108 


\section{List of Notations and Abbreviations}

\begin{tabular}{|c|c|}
\hline$\epsilon_{r}$ & Relative Dielectric Constant \\
\hline$\epsilon_{\text {eff }}$ & Effective Dielectric Constant \\
\hline$\lambda_{0}$ & Free Space Wavelength \\
\hline$\sigma$ & Standard Deviation \\
\hline ADS & Advanced Design System (Agilent Software) \\
\hline $\mathrm{AOA}$ & Angle of Arrival \\
\hline AR & Axial Ratio \\
\hline ASK & Amplitude Shift Keying \\
\hline $\mathrm{CRC}$ & Communications Research Center (Ottawa Canada) \\
\hline $\mathrm{DC}$ & Direct Current \\
\hline$D_{\mathrm{f}}$ & Loss Tangent \\
\hline DF & Direction Finding \\
\hline$D_{k}$ & Relative Dielectric Constant \\
\hline DOA & Direction of Arrival \\
\hline EIRP & Effective Isotropic Radiated Power \\
\hline EM & Electromagnetics \\
\hline EPC & Electronic Product Code \\
\hline FCC & Federal Communications Commission \\
\hline FET & Field-Effect Transistor \\
\hline HDX & Half Duplex \\
\hline $\mathrm{HF}$ & High Frequency \\
\hline HFSS & High Frequency Structure Simulator (Ansoft Software) \\
\hline
\end{tabular}




$\begin{array}{ll}\text { ISM } & \text { Industrial Scientific and Medical } \\ \text { LHCP } & \text { Left Hand Circular Polarization } \\ \mathrm{MHz} & \text { Megahertz } \\ \text { PCB } & \text { Printed Circuit Board } \\ \text { QFHA } & \text { Quadrifilar Helix Antenna } \\ \text { RF } & \text { Radio Frequency } \\ \text { RFID } & \text { Radio Frequency Identification } \\ \text { RHCP } & \text { Right Hand Circular Polarization } \\ \text { SMA } & \text { Sub-Miniature Version A (RF Connector) } \\ \text { UHF } & \text { Ultra High Frequency } \\ \text { VNA } & \text { Vector Network Analyzer }\end{array}$




\section{Chapter 1}

\section{Introduction}

\subsection{Motivation}

Although Radio Frequency Identification (RFID) has been around for decades recent advancements in the semiconductor industry have made low cost passive Ultra High Frequency (UHF) RFID tags a reality. Currently these RFID tags can be read from distances greater than 10 meters[1]. As the read distance continues to increase there is a growing demand for the accurate location of the tags. Localization using low cost RFID tags has many applications. Currently RFID based localization technology has low precision and limited read range. Both academia and industry are eagerly working to solve these deficiencies.

\subsection{Thesis Objectives}

A well known military direction finding method has been considered for locating RFID tags [2]. The proposed direction finding technique compares the received signals phase difference on two or more antennas to determine the Direction of Arrival (DOA). The phase comparison antenna array is commonly referred to as an interferometer array. An investigation of an interferometer array for UHF RFID is the objective of this thesis. The interferometer array should be low cost and provide good performance so as to be of commercial interest. There are no commercially available antennas in the UHF RFID frequency band that meet the bandwidth, radiation pattern, polarization, and size requirements of a direction finding RFID interferometer array. Therefore, a custom antenna element must be developed and evaluated. 


\subsection{Thesis Contributions}

A simple three element equilaterally spaced interferometer array has been considered and analyzed. Two different antenna elements have been developed, tested and implemented in the chosen interferometer configuration. First, a commonly used microstrip patch antenna was selected for the interferometer array. The microstrip patch antenna is low cost, meets the RFID performance requirements, and is aesthetically pleasing. The design of a traditional patch in an interferometer involved placing greater importance on maintaining very pure circular polarization. In addition it was necessary to miniaturize the patch in order to physically place the antennas in the proposed interferometer configuration. A second antenna, the Quadrifilar Helix Antenna (QFHA) has also been investigated for use in an RFID interferometer array. The QFHA is a nontraditional RFID antenna; however it has many performance benefits. Most notably, the antenna can be designed with a shaped radiation pattern that can increase the read range for this application. This antenna is a good candidate for RFID readers where the antenna is situated above the coverage area. The shaped beam antenna work has been published in two conference papers[3],[4]. This work has been presented at the Antennas and Applied Electromagnetics (ANTEM) Conference (Ottawa Canada, July 2010) as well as the Antennas and Propagation Society (APS) Symposium (Toronto Canada, July 2010).

\subsection{Thesis Organization}

This thesis is divided into seven chapters organized as follows:

Chapter 1 - Presents the motivation and objectives of the thesis.

Chapter 2 - Provides an understanding of UHF RFID with emphasis placed on the reader antenna. 
Chapter 3 - Presents the direction finding method using phase comparison and an analysis of the proposed interferometer configuration.

Chapter 4-Describes the theory, design, fabrication, and testing of the microstrip patch antenna. The testing and evaluation of the antenna in the interferometer array is also presented.

Chapter 5-Outlines the reasoning for choosing the nontraditional QFHA antenna. The theory, design, fabrication and testing of the QFHA and the testing and evaluation of the antenna in the interferometer array are presented.

Chapter 6-A discussion of the finding and results are given as well as the opportunities for future work. 


\section{Chapter 2}

\section{RFID Background}

\subsection{Introduction}

This chapter is divided into three major sections. First, the growing significance of UHF RFID is identified. Next, the concepts of UHF RFID are explained including backscatter radiation, the power source of passive RFID tags, and a brief description of the communication protocol. Finally, background information for UHF RFID reader antennas is given, including bandwidth, polarization, radiation patterns, and impedance matching. The RFID reader antenna topics are described in the greatest detail since they are the main focus of this work.

\subsection{The RFID Revolution}

Imagine a world where every manufactured object could be automatically identified without human intervention thanks to a unique Electronic Product Code (EPC). A standard infrastructure would evolve that could easily collect and share information about the location of these objects. This idea could be used to automate store checkouts which would mean an end to the lineups at the grocery store. The network would also know if stock was low or approaching expiration. Stock could then be automatically ordered to refill the shelves. It is an idea that lends itself to the creation and development of powerful supply management and marketing tools.

This vision was triggered by researchers at MIT, notably by David Brock, Sanjay Sarma, Sonny Siu, and Eric Nygren [1]. With support from additional universities and industry partners, the Auto-ID center was created in 1999. The Auto-ID center maintains that to enable billions of inexpensive objects to be automatically identified, RFID tags need to be as simple and as cheap as possible. Emphasis is placed on RFID technology in the $900 \mathrm{MHz}$ range since it offers the best 
compromise between cost, read range, and capability [1]. Two startup companies, Matrics and Alien were among the first to use the Auto-ID concepts to create the first generation of low cost passive UHF technology. Alien and Matrics UHF RFID systems are displayed in Figure 2.1 and Figure 2.2 respectively.

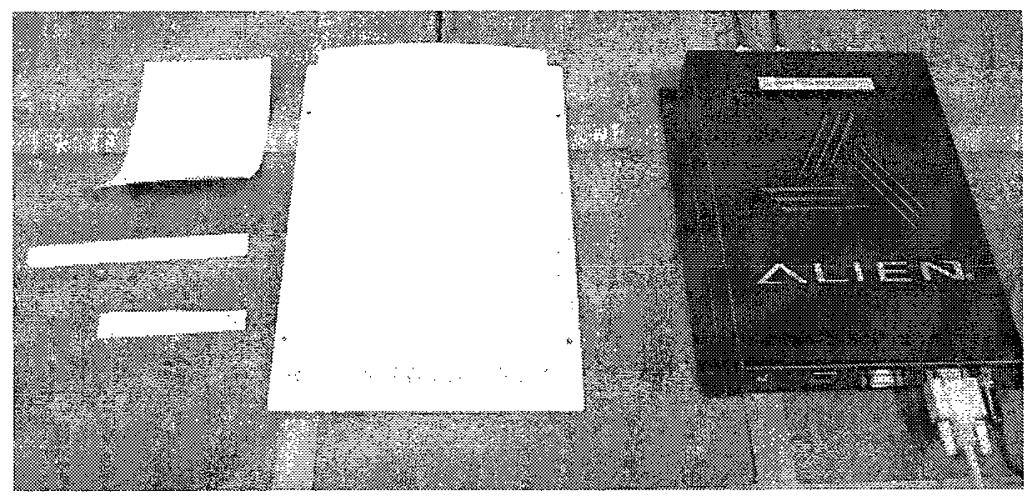

Figure 2.1: Alien Passive RFID Tags (Left) Antenna (Center) and Reader (Right) [5]

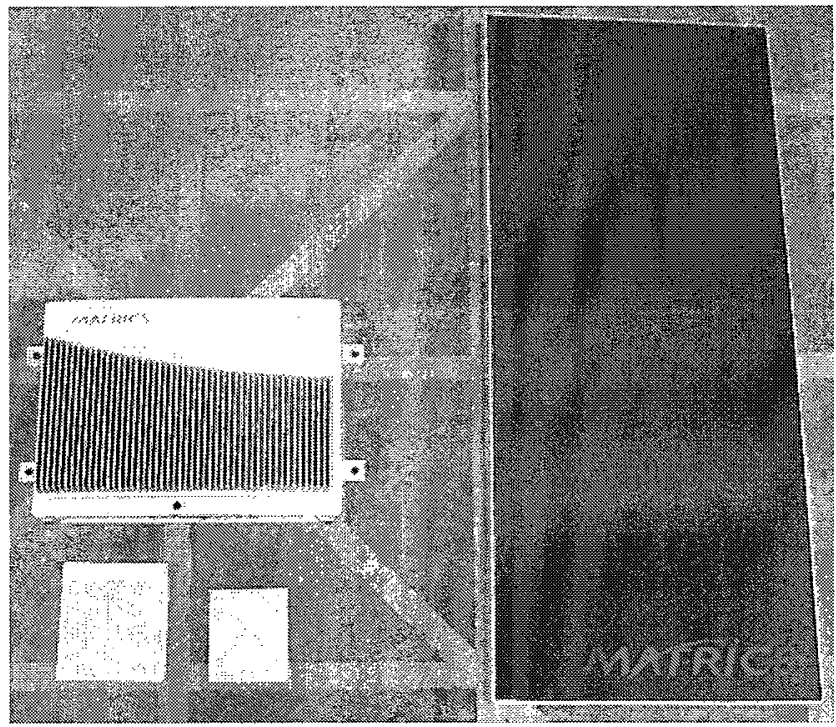

Figure 2.2: Matrics Passive RFID Tags (Bottom Left) Reader (Top Left) Antenna (Right) 
In 2003, [a nonprofit organization] EPC Global, was formed by the administrators of the international barcode system. The major project of EPC Global was to create a standard air interface. The current air interface is the globally accepted protocol for conversing with passive UHF RFID. The interface is called EPC Global Gen 2 (also referred to as ISO 18000-6c), and was finalized in 2005 .

Also in the 2005 time frame, the United States Department of Defense, Wal-Mart, Tesco, Metro, Target, and various other companies, made ground breaking announcements by mandating their top suppliers to provide RFID tags on pallets and cases. With the large scale production of RFID tags and readers, the cost of the technology is steadily dropping. The cost of a passive UHF RFID tag is anticipated to be less than 5 cents per tag in the near future [6].

\subsection{Understanding UHF RFID}

The roots of passive RFID can be traced back to WWII. German radar operators observed that when an aircraft was rolled the resulting signal backscattered from the aircraft would show up as blips on the radar operator's screen. The German air force decided to use this idea by training pilots to roll their aircraft when approaching friendly radar zones. This procedure gave the radar operators a means to determine if the approaching aircraft was a friend or foe. Changing the attitude of the aircraft was the method of modulating the backscattered signal in WWII as depicted in Figure 2.3. Similarly in passive RFID, the impedance of the tags antenna is changed so as to modulate the backscattered signal shown in Figure 2.4 . 


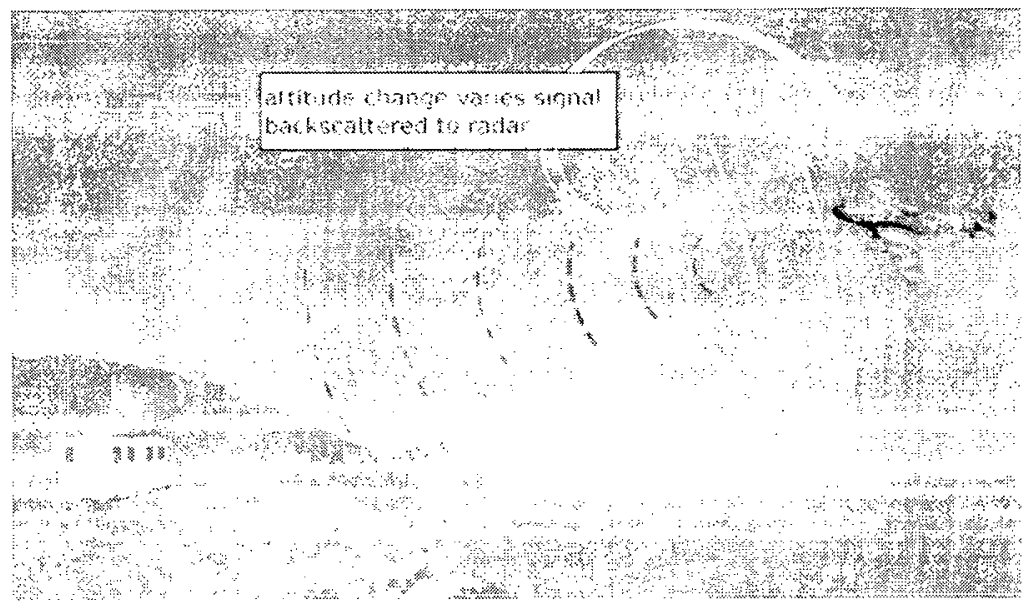

Figure 2.3: Use of Backscatter Radiation to Communicate with a Radar Operator [1]

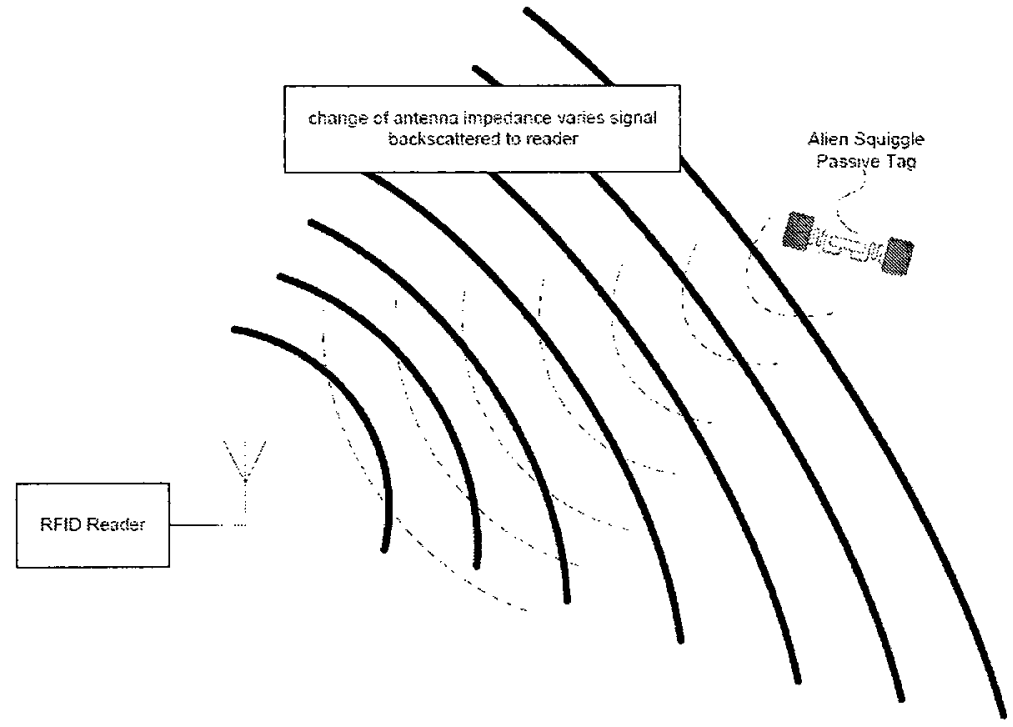

Figure 2.4: The use of Backscattered Radiation in Passive RFID

It should be noted that passive backscatter is a far field method and is a different concept than familiar magnetic coupling used on RFID technology such as smartcards, credit cards, and pay passes. Magnetic coupling has read range limited to a few feet while passive UHF tags can be read at distances greater than 10 meters. 
A typical RFID system consists of a computer, RFID reader, reader antenna, and an RFID tag as shown in Figure 2.5.



Figure 2.5: Typical Stationary RFID System [5]

The computer controls the readers operation and also stores and displays the readers output data.

The RFID reader is responsible for transmitting a signal to the RFID tag. The RFID reader transmits a signal and receives the tags backscatter through the reader antenna. In UHF RFID, the reader continuously sends out an RF wave around $900 \mathrm{MHz}$, which provides the carrier signal for the reader as well as the tag. This continuous wave is also responsible for energizing the tag. The reader and tag communicate with a Half Duplex (HDX) protocol where the reader and tag take turns communicating. As Figure 2.6 illustrates, the continuous wave constantly provides energy transfer while the reader and tag alternate transmitting data. 


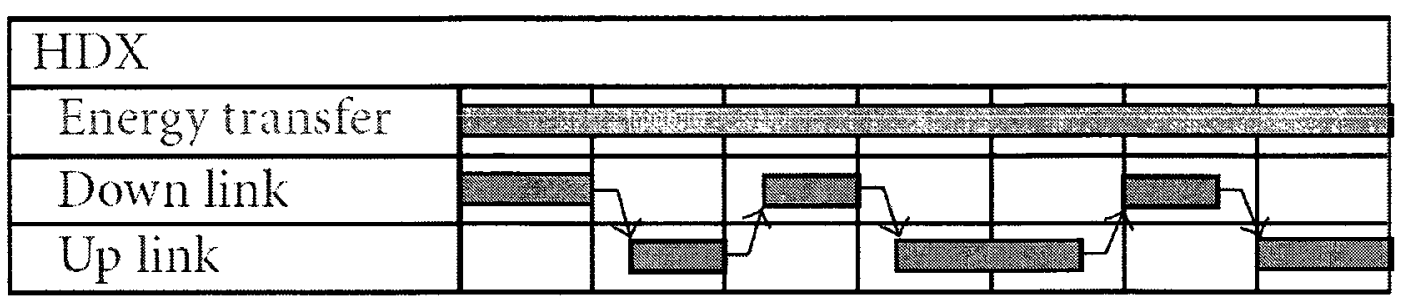

Figure 2.6: HDX RFID Communication Protocol

The reader communicates to the tag using Amplitude Shift Keying (ASK) modulation as shown in Figure 2.7 (a).

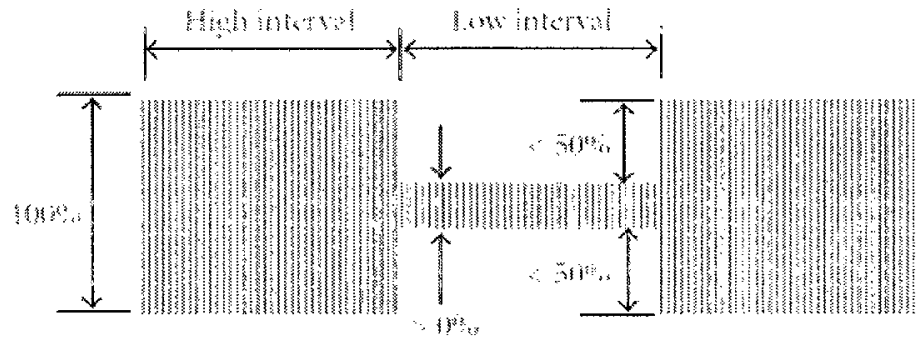



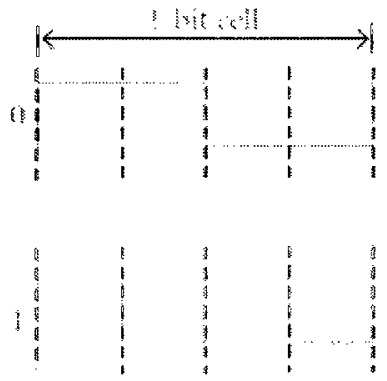

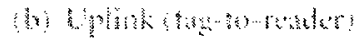

Figure 2.7: Examples of Typical RFID Modulation [2] 
The tag replies to the reader by backscattering the continuous wave signal using pulse interval encoding shown in Figure 2.7 (b). In pulse interval encoding, a binary zero is represented by two transitions in the bit interval, and a binary one is represented by four transitions.

A simple schematic of an RFID tag can be seen in Figure 2.8.

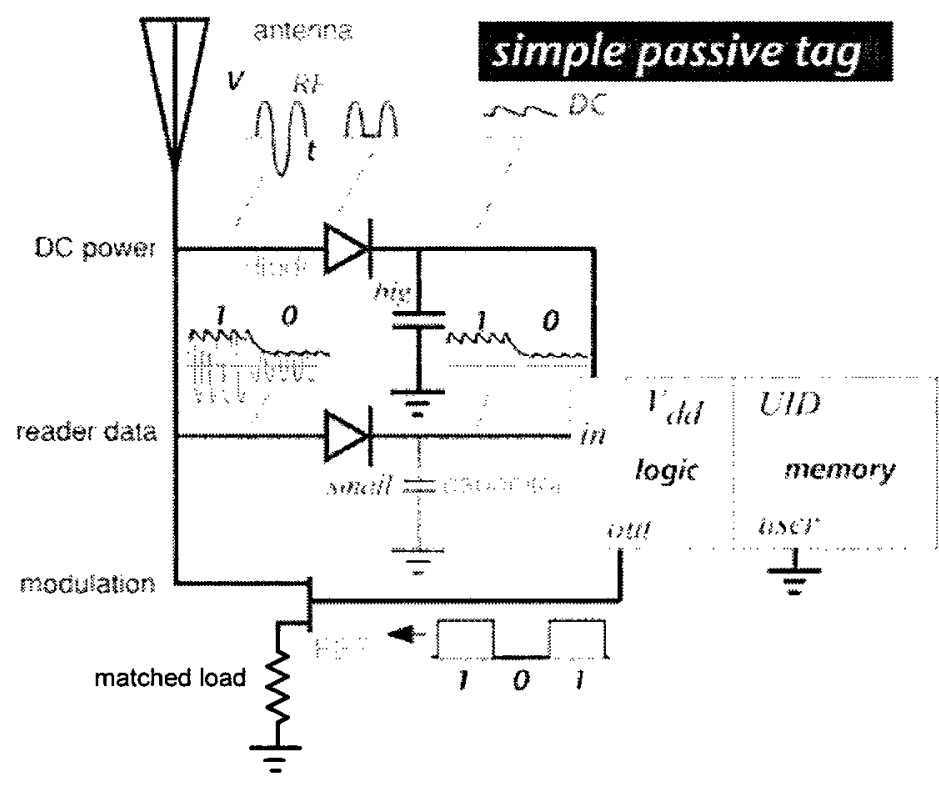

Figure 2.8: Simple Passive RFID Tag[1]

The continuous wave from the reader is used by the DC path to power the tags circuitry. The signal is rectified in the DC power path and then smoothed by the 'big capacitor' creating a stable DC power source. For a more simplistic understanding, consider a reader sending out a $900 \mathrm{MHz}$ continuous wave that is ASK modulated, querying the RFID tag for its EPC number. The DC power path retains its stable voltage because the "big capacitor" does not fully discharge when the reader sends a zero with low amplitude; however, in the data demodulation path, the 'small capacitor' does discharge when a zero is sent. The resulting analog signal in the demodulation path is then decoded and the reader data is processed by the logic circuitry. After deciphering the 
readers request for the tags EPC number, the tag then sends its EPC data signal to the Field-Effect Transistor (FET), turning it on and off creating the pulse interval encoded backscatter response.

When the FET is on, the antenna is connected to ground, whereas when the FET is off, the antenna is essentially open. The backscatter mechanism is illustrated in Figure 2.9.



Figure 2.9: Simplified Physics of Backscatter Signaling [1]

When the antenna is connected to ground, the readers continuous wave is backscattered, yet there is little backscatter when the antenna is open. By using this phenomenon the tag can respond to the readers query by replying with its EPC number. The reader antenna picks up the tags backscatter response and the reader deciphers and processes the signal. The received EPC number can then be sent to the computer and output to the user.

Backscatter modulation allows for a very simple RFID tag that does not require an oscillator or battery. This short explanation gives a basic understanding of passive UHF RFID. Detailed 
information of the communication protocol, and tag/reader operation is contained in [1],[5], and [7].

\subsection{The Reader Antenna}

There are several unique requirements of an RFID antenna at the reader. An antenna for UHF RFID must provide circular polarization, sufficient bandwidth, adeguate gain, and high isolation between transmit and receive signais. Commerciai RFID reader antennas are based upon the microstrip patch antenna seen in Figure 2.10.
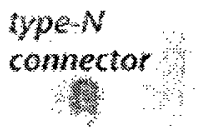

plastic radome

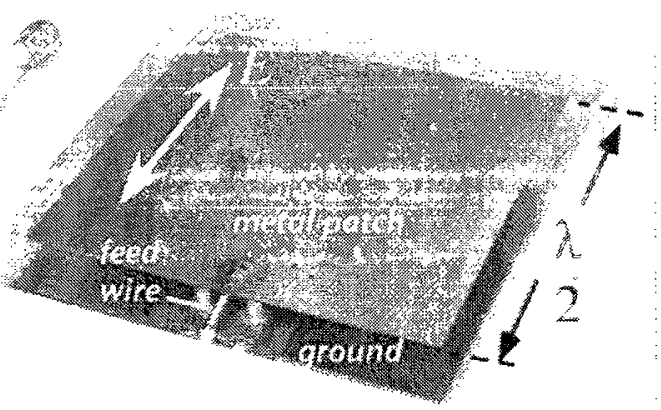

Br coble

Figure 2.10: Patch Antenna, with Plastic Radome (Left) and without Radome (Right) [1]

Not only is the patch antenna capable of meeting the performance requirements, the patch is low cost, and low profile making it aesthetically pleasing and easy to mount on a flat surface. The patch antenna is used with every major traditional RFID system in the market today.

\subsubsection{Frequency of Operation}

An efficient antenna must maintain adequate impedance matching, polarization purity, and pattern shape over the UHF frequency band. The frequency of operation of UHF RFID varies 
when traveling around the world since countries have made different choices concerning the frequencies where wireless devices operate. For example, in North America the allotted spectrum is $902-928 \mathrm{MHZ}$, while in Europe it is $865-868 \mathrm{MHz}$, and in Japan $952-954 \mathrm{MHz}$ is used. The reason for this is that countries have made different choices as to which frequencies certain wireless devices are allowed to operate at. This work will focus on North America where the spectrum of UHF RFID is $902-928 \mathrm{MHz}$. This is an unlicensed Industrial Scientific and Medical (ISM) band set aside by the Federal Communications Commission (FCC). The unlicensed operation of RFID in this band is subject to several limitations such as maximum output power, antenna gain, and certain frequency hopping behavior in order to reduce interference to other users.

\subsubsection{Radiation Pattern}

The radiation pattern of an antenna describes the spatial distribution of radiated energy. Antennas have a radiation pattern defined by the gain at azimuth and elevation angles. A patch antenna with corresponding azimuth and elevation angles is seen in Figure 2.11. 


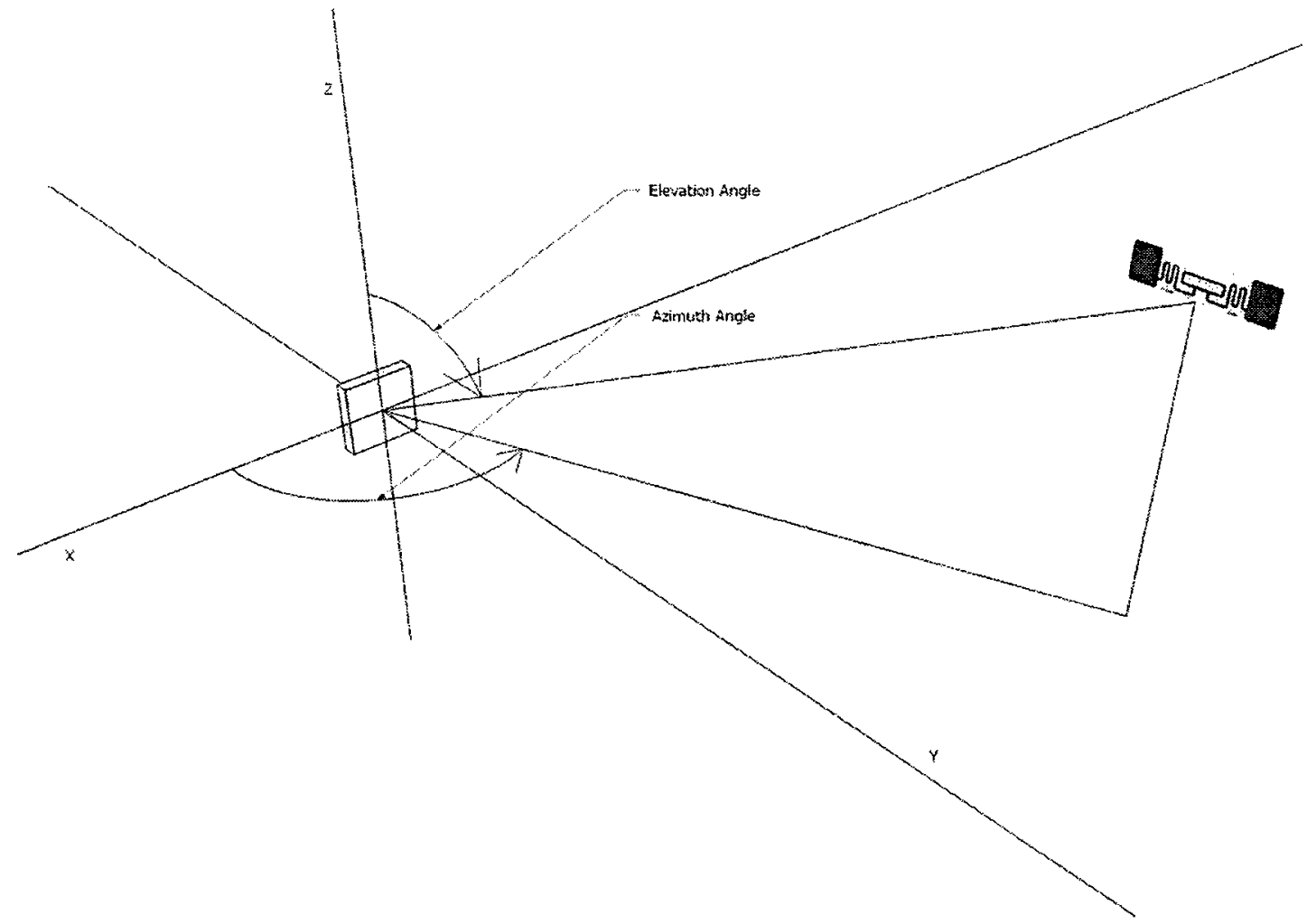

Figure 2.11: Antenna Elevation and Azimuth Angles

A radiation pattern is a 3-D plot of the antenna gain. The gain directed towards the RFID tag in Figure 2.11 can be determined by knowing the angles and the radiation pattern. A 3-D pattern is difficult and time consuming to plot; therefore, two 2-Dimensional cuts are usually given. These 'cuts' are in the elevation and azimuth planes. Since radiation patterns are generally symmetrical, the elevation and azimuth cuts normally contain all the necessary information for the full 3-D pattern.

Most RFID antennas have a peak gain around $6 \mathrm{~dB}$ which is due to regulatory reasons. The FCC mandates the output power of an RFID reader to be less than 1 Watt $(30 \mathrm{dBm})$. The FCC also limits the Effective Isotropic Radiated Power (EIRP) to a maximum of 4 Watts ( $36 \mathrm{dBm}$ ). Most 
RFID readers operate at the 1 Watt maximum so that the best read distance and performance can be achieved. Therefore the antenna is usually limited to a gain of less than $6 \mathrm{~dB}$.

\subsubsection{Polarization}

Antenna polarization describes the orientation of the electric field radiated by an antenna. An intuitive example is a simple dipole antenna. Current only flows down the axis of a simple dipole, which radiates a wave polarized in this direction only. No signal can be received from a tag when the reader antenna has the opposite polarization. This situation is explained in Figure 2.12 where maximum energy is transferred when the antennas are parallel but no energy is transferred when the antennas are orthogonal.
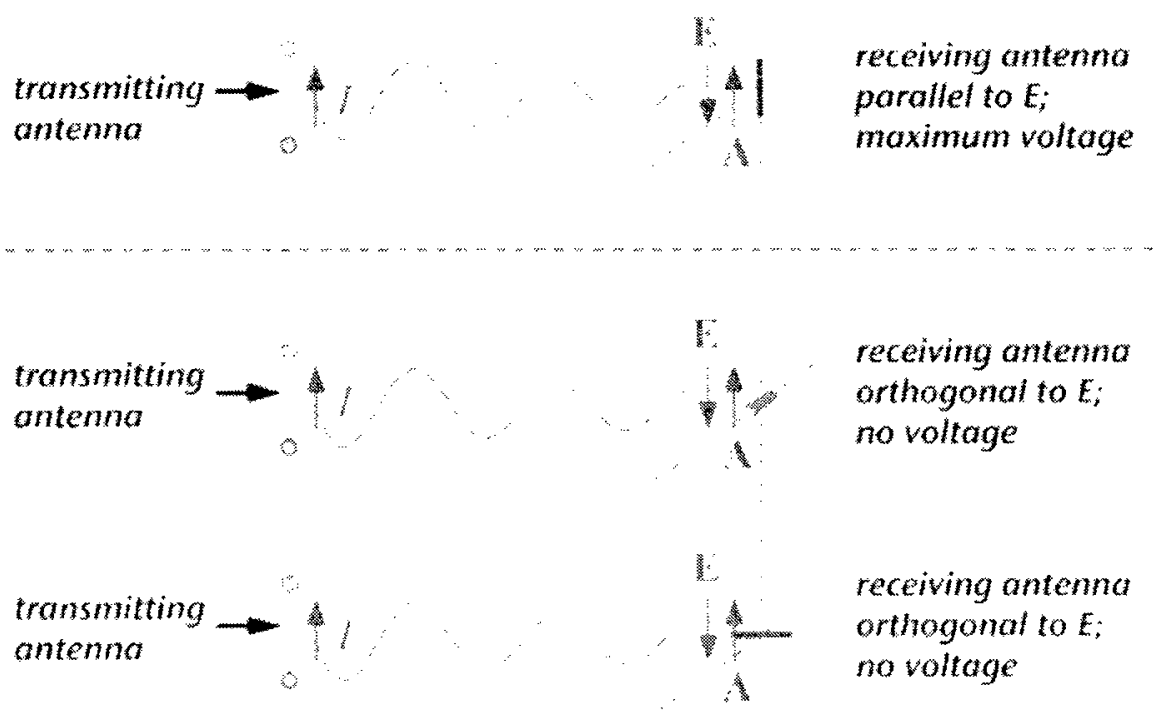

Figure 2.12: Simple Dipole Polarization Example [1]

Since tag antennas are typically linearly polarized dipoles that have unknown orientation, it is necessary to use a reader antenna which is insensitive to polarization mismatch. The solution is to use a circularly polarized antenna. 


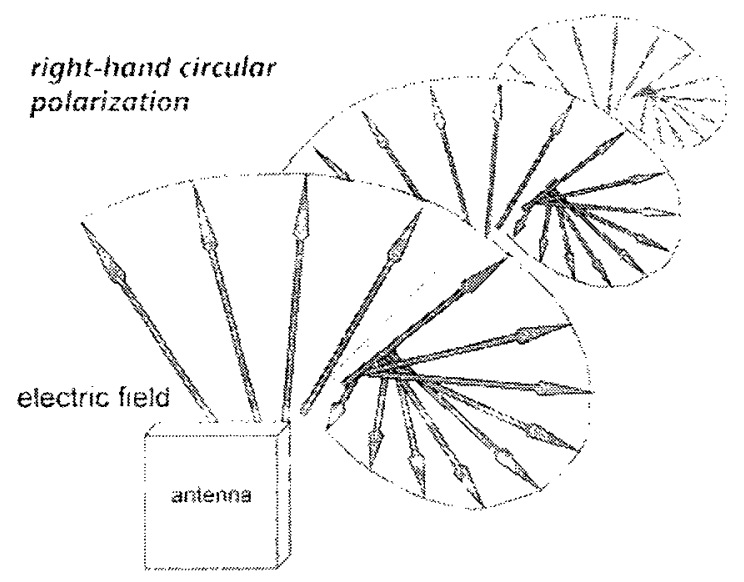

Figure 2.13: Circular Polarization [1]

As seen in Figure 2.13 the electric field rotates from vertical to horizontal in time with the RF cycle. An antenna with circular polarization can utilize both vertical and horizontal polarization. Since RFID antennas are normally used in applications where the orientation of the tag is unknown it is necessary to have a circularly polarized reader antenna.

\subsubsection{Impedance Match}

In North America UHF RFID is designated to operate in the range $902-928 \mathrm{MHz}$. An antenna must efficiently radiate over this bandwidth. Antennas are usually connected with a $50 \Omega$ transmission line. Maximum power transfer occurs when the impedance seen looking towards the source is the same as the impedance looking towards the load (antenna). The impedance of an antenna changes with frequency and the goal of antenna design is to maintain a $50 \Omega$ impedance over the frequency of interest.

RFID readers often use a 'monostatic configuration', which means the same antenna is used for transmitting and receiving the signal. Since the signals transmitted and received by the reader are 
at exactly the same frequency, it is difficult to filter them. This makes the impedance match especially important. When the antenna is poorly matched, transmit power is reflected back from the antenna and can drown out the tiny signal backscattered from the tag.

\subsection{Conclusion}

UHF RFID is an exciting field that is rapidly growing and is the topic of extensive amounts of research. This chapter gives a basic understanding of UHF RFID technology. The UHF RFID antenna was described in greater detail. The reasoning for the antennas frequency of operation, polarization, and importance of impedance matching has been discussed. These concepts are relevant to the antenna designs presented in this work. 


\section{Chapter 3}

\section{Direction of Arrival using Phase Comparison}

\subsection{Introduction}

Finding the Direction of Arrival (DOA) of an incoming signal is extensively used in electronic warfare to find enemy's transmitting communications and radar signals. There are several techniques that can be used for DOA measurements which include mechanically rotating a directional antenna or electronically steering a phased array antenna. Another popular method used to find DOA a is phase comparison of two or more antenna elements. An antenna array used for phase comparison is often called an interferometer array and an example is shown in Figure 3.1. The array below is designed for use over a broad bandwidth and uses different separations of the antennas to resolve ambiguities.

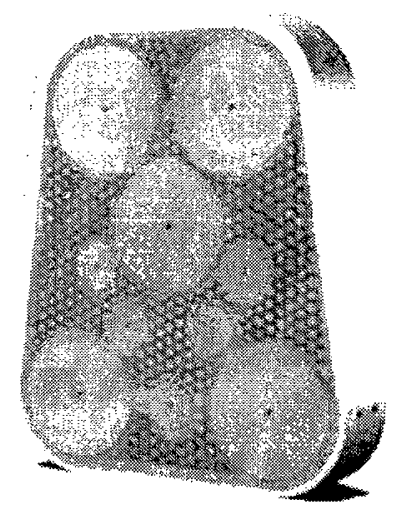

Figure 3.1: Interferometer Array [8]

Most military applications require extremely wide bandwidth interferometers since the incoming signal's frequency is usually unknown. An interferometer for UHF RFID does not have the 
difficult bandwidth requirements that a military system does. In UHF RFID the frequencies are well known and the band is relatively narrow.

Phase comparison using an interferometer array was chosen for this work since it involves relatively small antenna elements and a simple antenna array that does not need to be rotated. Keeping the direction finding solution simple and low cost is a priority for a commercial RFID application. Phase comparison based direction estimation for passive RFID tags have been proposed by Y. Zhang, M. Amin, and S. Kaushik in 2007 [2]. The publication looks at direction finding of RFID tags using a simple two element antenna array. It was concluded in [2] that the phase comparison method is a viable solution and is capable of achieving a high DOA accuracy. Phase based methods are also proposed for RFID in [9] where it is emphasized that phase based methods are capable of high accuracy and are immune to variations in signal strength. However, phase comparison techniques are susceptible to phase distortion.

This chapter provides a basic understanding of the phase comparison method for DOA estimation. A three element interferometer was chosen for this work, and the mathematics behind the interferometer is described in detail. Finally an analysis of the phase deviation versus DOA estimation accuracy is given in the last section.

\subsection{DOA Phase Comparison Basics}

To understand the principles of an interferometer array, consider the two element interferometer in Figure 3.2. 


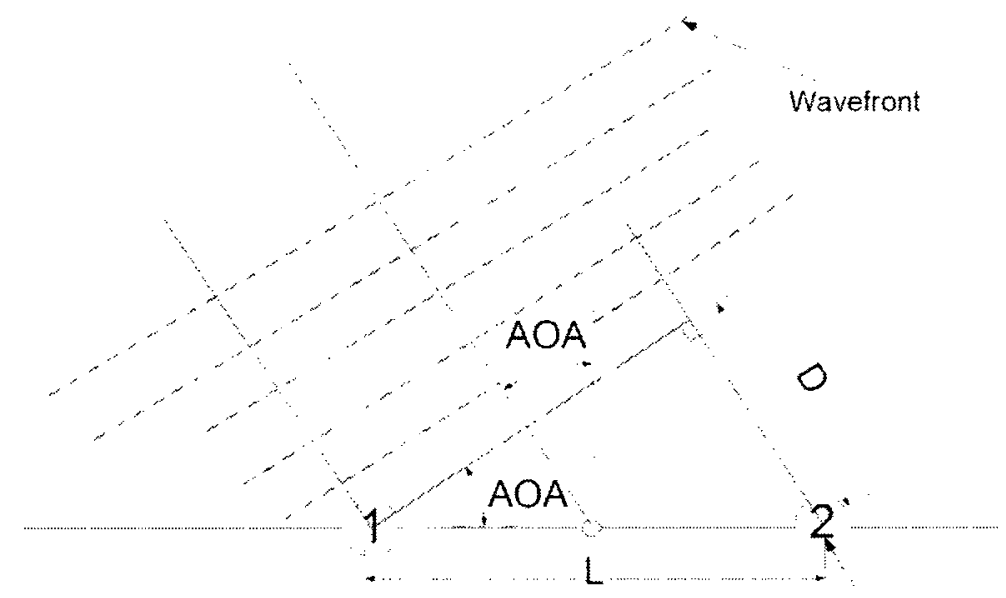

Figure 3.2: Two Element Interferometer

The Angle of Arrival (AOA) is the angle with respect to the antenna array that the signal is propagating from. In Figure 3.2 it is assumed that the RFID tag is sufficiently far away and the incoming signal is approximated by a perfectly parallel wavefront. The phase difference between the two antennas is measured and used to calculate the difference in distance (D).

$$
D=L \cdot \sin ((A O A)
$$

$\mathrm{L}$ is the length separating the two antennas and the distance $D$ is calculated from the phase difference $\phi_{12}$ by Equation 3.2.

$$
D=\frac{\phi_{12} \lambda}{360^{\circ}}
$$

$\lambda$ is the wavelength of the carrier frequency. By combining Equations 3.1 and 3.2 the AOA can be determined from the phase difference with Equation 3.3.

$$
A O A=\arcsin \left(\frac{\phi_{12} \lambda}{360^{\circ} L}\right)
$$

There are two types of ambiguities in the AOA measurement that can occur for an interferometer, these ambiguities are called long baseline and mirror image ambiguities. 


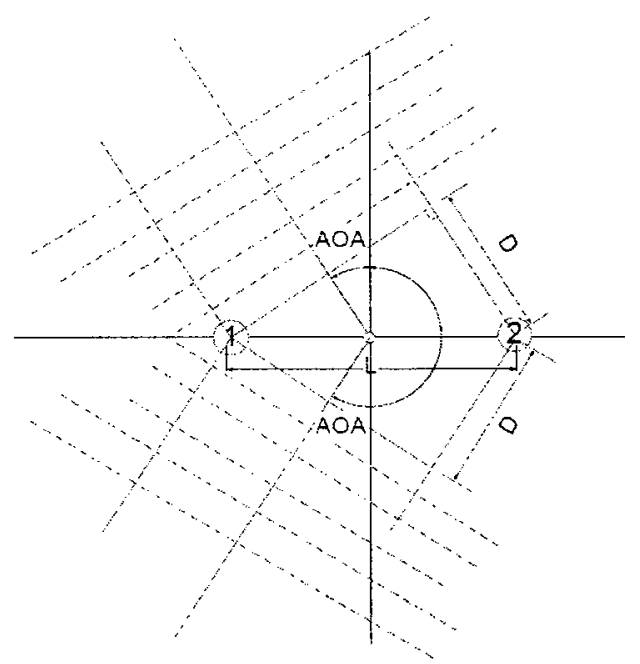

Figure 3.3: Mirror Image Ambiguity for a Two Element Interferometer

Figure 3.3 illustrates the mirror image ambiguity. The received phase at each antenna is identical whether the signal arrives from the north-west or the south-west. In a simple two element interferometer there will always be a mirror image ambiguity.

Long baseline ambiguities occur when the distance $D$ has an electrical length larger than 360 degrees. No long baseline ambiguities occur when an antenna separation less than or equal to a half wavelength is used since the phase difference swing is less than 360 degrees. A plot depicting the AOA versus the measured phase difference for a two element interferometer is shown in Figure 3.4. 


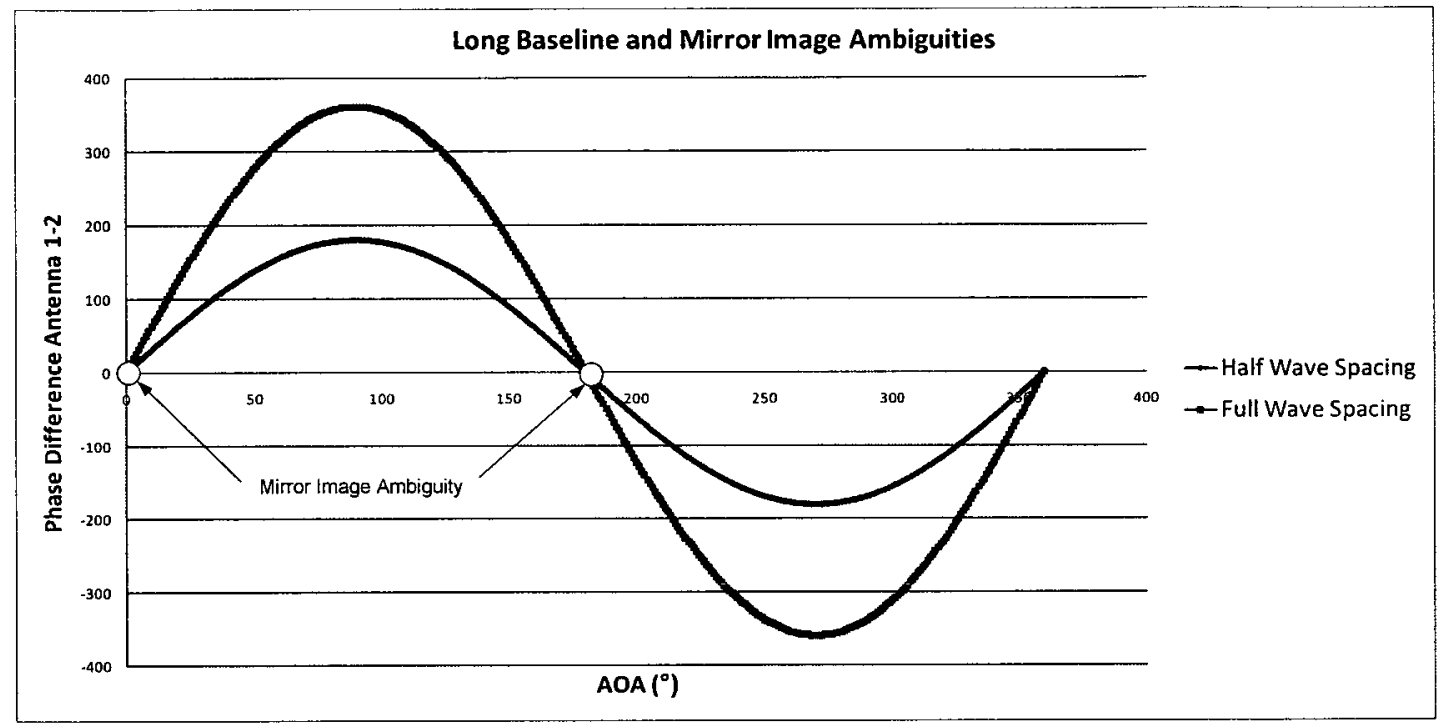

Figure 3.4: Phase Difference as a Function of AOA for a Two Element Interferometer

Figure 3.4 shows the phase difference versus azimuth angle for a half and full wavelength spaced interferometer. The full wavelength interferometer has a phase difference that swings $\pm 360^{\circ}$ creating ambiguous phase differences (long baseline ambiguities). The half wavelength spaced interferometer corresponds to a phase change of $\pm 180^{\circ}$ and no long baseline ambiguities occur. Any spacing that creates a phase difference change larger than $360^{\circ}$ will cause long baseline ambiguities. Also notice that the mirror image ambiguities (for example at $0^{\circ}$ and $180^{\circ}$ ) occur regardless of the antenna separation.

An interesting note is that the steeper the rate of change of phase difference versus AOA the more accurate the direction estimation is. The full wavelength spacing produces a steeper rate of change and it is intuitive that the larger antenna separations produce a more accurate AOA estimation. Notice that the rate of change is steepest around boresight at $0^{\circ}$ and $180^{\circ} \mathrm{AOA}$, which is the most accurate area. The accuracy of the system will be worst at $90^{\circ}$ and $270^{\circ}$ AOA since the rate of change is lowest in these areas. While larger antenna separations provide a more 
accurate system, they also introduces ambiguities. When a very accurate system is required it is common to use a half wavelength spacing along with larger spacing. The half wavelength spacing is used to resolve the ambiguities while larger spacing increases accuracy.

The phase comparison theory also holds true in three dimensions with an added variable $\left(\mathrm{AOA}_{\text {Elevation }}\right)$ to account for the elevation angle. The array in three dimensions can be seen in

Figure 3.5.

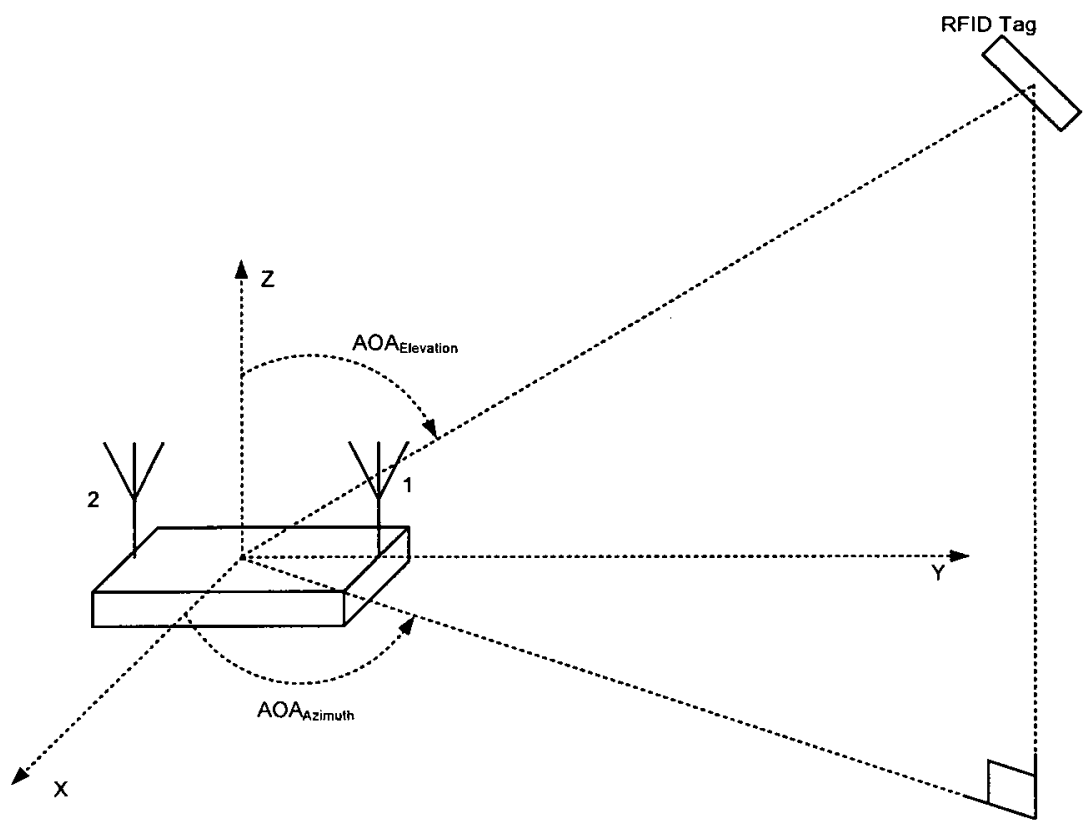

Figure 3.5: Phase Comparison in Three Dimensions

The elevation angle reduces the accuracy of the $\mathrm{AOA}_{\text {Azimuth }}$ estimation. An elevation angle can be thought of a reduction of the effective length separating the two antennas. 


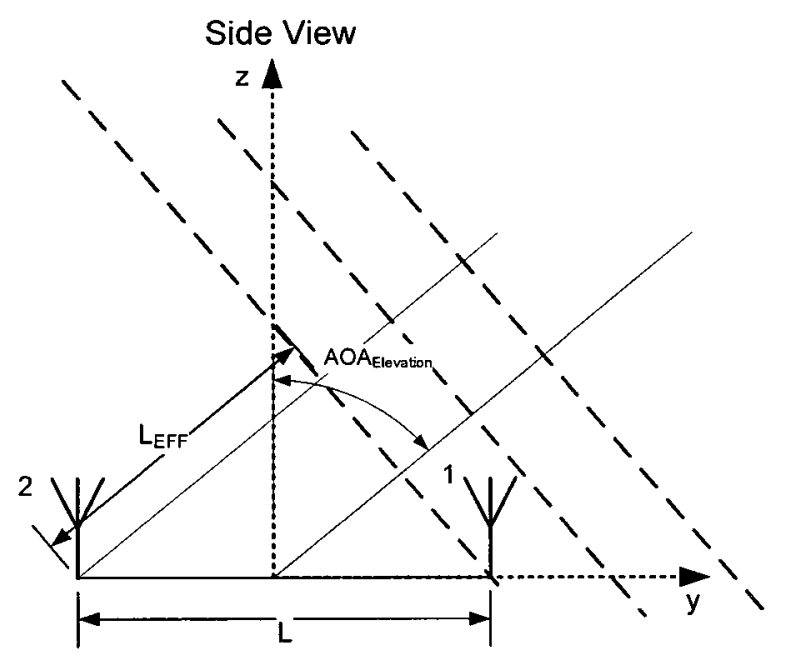

Figure 3.6: Elevation Angle and Effective Separation of the Antennas

The effective length from Figure 3.6 can be calculated from Equation 3.4.

$$
L_{E F F}=L \cdot \sin \left(\left(A O A_{\text {Elevation }}\right)\right.
$$

The effective length caused by the elevation angle then modifies the distance Equation 3.2 into Equation 3.5 below.

$$
D=L \cdot \sin \left(A O A_{\text {Elevation }}\right) \cdot \sin \left(\left(A O A_{\text {Azimut } h}\right)\right.
$$

Then by substituting Equation 3.4 into equation 3.5 and rearranging, the $\mathrm{AOA}_{\text {Azimuth }}$ is obtained.

$$
A O A_{\text {Azimut } h}=\arcsin \left(\frac{\phi_{a b} \cdot \lambda}{360^{\circ} \mathrm{L} \cdot \sin !\left(4 O A_{\text {Elevation }}\right)}\right)
$$

Equation 3.6 describes how the elevation angle reduces the accuracy of the $\mathrm{AOA}_{\text {Azimuth }}$ calculation.

\subsubsection{Three Element Interferometer Array}

There are two major disadvantages to using a two element interferometer. The first is that the signals cannot be resolved in three dimensions because of the two unknowns; the $\mathrm{AOA}_{\mathrm{Azimuth}}$ and the $\mathrm{AOA}_{\text {Elevation. }}$ Also the interferometer can only be used to locate tags in a 180 degree field of 
view because of the mirror image ambiguity. A three element interferometer has been chosen for this work since it is capable of resolving these issues.

\subsection{Proposed Three Element Interferometer}

The three element interferometer in an equilateral configuration as shown in Figure 3.7 has been chosen for this design, because it is simple and uses a minimum number of elements for $360^{\circ}$ azimuth coverage. The elements have been spaced $16 \mathrm{~cm}$ apart so that no ambiguities arise when determining DOA in the $902-928 \mathrm{MHz}$ band. The mathematics behind this interferometer will be described in this section. The math behind the array is derived in this section, based off the work by Roger Edwards in [10].

\subsubsection{Mathematics of a Three Element Equilateral Triangle Interferometer}

A three element interferometer array in an equilateral triangle configuration is depicted in Figure 3.7. 

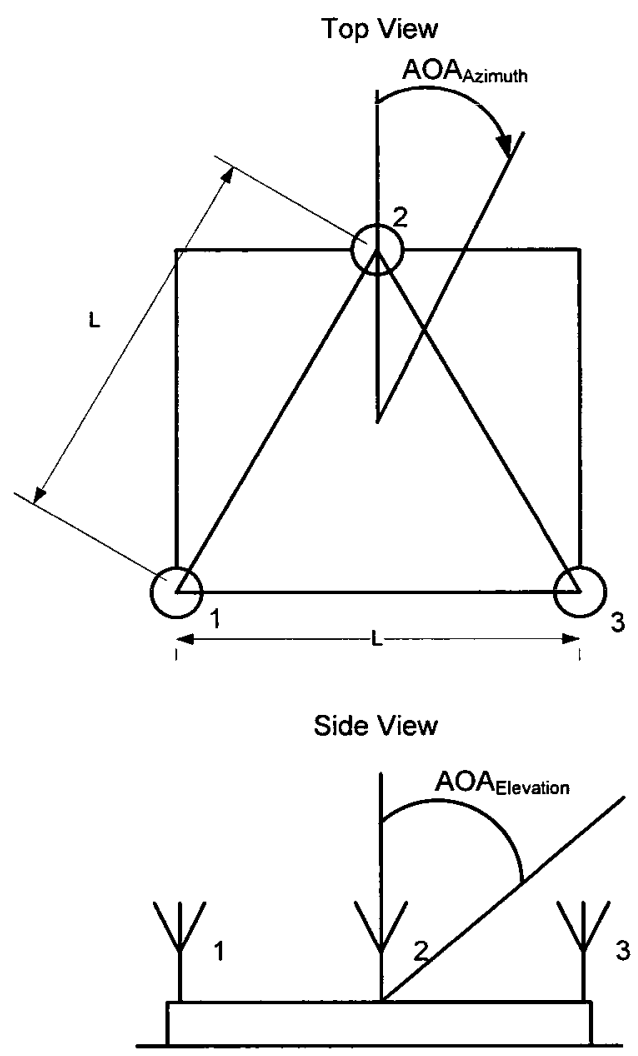

Figure 3.7: Three Element Interferometer in an Equilateral Triangle Configuration

Similar to the two element interferometer, the AOA can be derived from the geometry just as was done in the two element case. The distance differences are written as follows:

$$
\begin{aligned}
& D_{21}=L \cdot \sin \left(( A O A _ { \text { Elevation } } ) \cdot \operatorname { c o s } \left(\left(A O A_{\text {Azimut } h}-30^{\circ}\right)\right.\right. \\
& D_{31}=L \cdot \sin \left(( A O A _ { \text { Elevation } } ) \cdot \operatorname { c o s } \left(\left(A O A_{\text {Azimut } h}-90^{\circ}\right)\right.\right. \\
& D_{23}=L \cdot \sin \left(( A O A _ { \text { Elevation } } ) \cdot \operatorname { c o s } \left(\left(A O A_{\text {Azimut } h}+30^{\circ}\right)\right.\right.
\end{aligned}
$$

Using Equation 3.2 the phase differences can be related to the distance differences. 


$$
\begin{aligned}
& \phi_{21}=\frac{L \cdot 360^{\circ}}{\lambda} \cdot \sin \left(A O A_{\text {Elevation }}\right) \cdot \cos \left(A O A_{\text {Azimut } h}-30^{\circ}\right) \\
& \phi_{31}=\frac{L \cdot 360^{\circ}}{\lambda} \cdot \sin \left(A O A_{\text {Elevation }}\right) \cdot \cos \left(A O A_{\text {Azimut } h}-90^{\circ}\right) \\
& \phi_{23}=\frac{L \cdot 360^{\circ}}{\lambda} \cdot \sin \left(A O A_{\text {Elevation }}\right) \cdot \cos \left(A O A_{\text {Azimut } h}+30^{\circ}\right)
\end{aligned}
$$

The $\mathrm{AOA}_{\mathrm{Azimuth}}$ can be solved from Equations 3.10, 3.11, and 3.12. The trigonometry identity of Equation 3.13 will be used to transform Equations 3.10, 3.11, and 3.12 into the three relations $3.14,3.15$, and 3.16 .

$$
\begin{gathered}
\cos (a+b)=\cos ((a) \cdot \cos ((b)-\sin (a) \cdot \sin (b) \\
\phi_{21}=\frac{L \cdot 360^{\circ}}{\lambda} \cdot \sin \left(A O A_{\text {Elevation }}\right) \cdot\left[\cos \left(A O A_{\text {Azimut } h}\right) \cos \left(30^{\circ}\right)+\sin \left(A O A_{\text {Azimut } h}\right) \sin \left(30^{\circ}\right)\right] \\
\phi_{31}=\frac{L \cdot 360^{\circ}}{\lambda} \cdot \sin \left(A O A_{\text {Elevation }}\right) \cdot\left[\cos \left(A O A_{\text {Azimut } h}\right) \cos (90)+\sin \left(A O A_{\text {Azimut } h}\right) \sin (90)\right] \\
\phi_{23}=\frac{L \cdot 360^{\circ}}{\lambda} \cdot \sin \left(A O A_{\text {Elevation }}\right) \cdot\left[\cos \left(A O A_{\text {Azimut } h}\right) \cos (30)-\sin \left(A O A_{\text {Azimut } h}\right) \sin ((30)]\right.
\end{gathered}
$$

By adding Equations 3.14 and 3.16, Equation 3.17 is obtained:

$$
\phi_{21}+\phi_{23}=\frac{2 \cdot L \cdot 360^{\circ}}{\lambda} \cdot \sin \left(A O A_{\text {Elevation }}\right) \cdot \cos \left(A O A_{\text {Azimut } h}\right) \cos \left(30^{\circ}\right)
$$

Then by dividing Equation 3.15 by Equation 3.17, the result is Equation 3.18 . 


$$
\frac{\phi_{31}}{\phi_{21}+\phi_{23}}=\frac{\tan \left(A O A_{\text {Azimut } h}\right)}{2 \cos \left(\left(30^{\circ}\right)\right.}
$$

Equation 3.18 can then be manipulated and solved in terms of $\mathrm{AOA}_{\mathrm{Azimuth}}$ to create Equation 3.19. Equation 3.19 provides a calculation of $\mathrm{AOA}_{\text {Azimuth }}$ without the need for $\mathrm{AOA}_{\text {Elevation }}$.

$$
A O A_{\text {Azimut } h}=\arctan \left(2 \cdot \cos \left(30^{\circ}\right)\left(\frac{\phi_{31}}{\phi_{21}+\phi_{23}}\right)\right)
$$

$A O A_{\text {Elevation }}$ can be solved from Equations 3.10,3.11, and 3.12 to obtain Equation 3.23. By squaring Equation 3.17 and squaring and scaling Equation 3.15, Equations 3.20 and 3.21 are created.

$$
\begin{gathered}
\left(\phi_{21}+\phi_{23}\right)^{2}=\left(2 \cdot \cos \left(\left(30^{\circ}\right)\right)^{2}\left(\frac{L \cdot 360^{\circ} \cdot \sin \left(A O A_{\text {Elevation }}\right)}{\lambda}\right)^{2} \cdot \cos ^{2}\left(A O A_{\text {Azimut } h}\right)\right. \\
\left(2 \cdot \cos \left(30^{\circ}\right) \phi_{31}\right)^{2}=\left(2 \cdot \cos \left(\left(30^{\circ}\right)\right)^{2}\left(\frac{L \cdot 360^{\circ} \cdot \sin \left(A O A_{\text {Elevation }}\right)}{\lambda}\right)^{2} \cdot \sin ^{2}\left(A O A_{\text {Azimut } h}\right)\right.
\end{gathered}
$$

Equation 3.22 is produced by adding Equations 3.20 and 3.21 then using Pythagorean identity to simplify the expression.

$$
\left(\phi_{21}+\phi_{23}\right)^{2}+\left(2 \cdot \cos \left((30) \phi_{31}\right)^{2}=(2 \cdot \cos !(30))^{2}\left(\frac{L \cdot 360^{\circ} \cdot \sin \left(A O A_{\text {Elevation }}\right)}{\lambda}\right)^{2}\right.
$$

Equation 3.22 can be manipulated to obtain $A O A_{\text {Elevation }}$ in terms of the phase differences in Equation 3.23.

$$
A O A_{\text {Elevation }}=\arcsin \sqrt{\frac{\left(\phi_{b c}+\phi_{b a}\right)^{2}+\left(2 \cdot \cos (30) \cdot \phi_{c a}\right)^{2}}{\left(2 \cdot \cos \left((30) \cdot 360 \cdot \frac{L}{\lambda}\right)^{2}\right.}}
$$

From the above mathematical explanation of the three element interferometer of Figure 3.7 it is clear that the phase differences can be used to obtain both $\mathrm{AOA}_{\text {Azimuth }}$ and $\mathrm{AOA}_{\text {Elevation }}$. It 
should be noted that Equations 3.19 and 3.23 provide AOA estimation for the proposed

interferometer. However because of the nature of the $\arcsin ()$ and $\arctan ()$ functions they cannot give unambiguous answers in the full $360^{\circ}$. A look up table is suggested for calculating the AOA if a full $360^{\circ}$ field of view is required.

\subsubsection{Effect of Phase Error on DOA Estimation}

The elevation angle of the arriving signal has a noticeable effect on the accuracy of the interferometer. Monte Carlo simulations have been performed at elevation angles of 15, 30, 60 and 90 degrees to determine the accuracy of the interferometer. The phase error at each antenna was given a unique random Gaussian distribution with either a $5^{\circ}$ or $10^{\circ}$ standard deviation. One hundred iterations of each simulation were performed to find the probable error of the DOA estimation. The results of the simulations are shown in Table 3.1. 
Table 3.1: Effect of Phase Error and Elevation Angle on DOA Estimation Accuracy (Monte Carlo Simulation of Proposed Three Element Interferometer)

\begin{tabular}{|c|c|c|c|}
\hline Simulation & $\begin{array}{c}\text { Elevation Angle } \\
\left({ }^{\circ}\right)\end{array}$ & $\begin{array}{c}\text { Phase Error } \sigma \\
\left({ }^{\circ}\right)\end{array}$ & $\begin{array}{c}\text { DOA Error } \sigma \\
\left({ }^{\circ}\right)\end{array}$ \\
\hline 1 & 90 & 5 & 1.54 \\
\hline 2 & 75 & 5 & 1.54 \\
\hline 3 & 60 & 5 & 1.93 \\
\hline 4 & 45 & 5 & 2.49 \\
\hline 5 & 30 & 5 & 2.96 \\
\hline 6 & 15 & 5 & $\underline{6.07}$ \\
\hline 7 & 0 & 5 & $\underline{\text { INFINITE }}$ \\
\hline 8 & 90 & 10 & 3.04 \\
\hline 9 & 75 & 10 & 3.26 \\
\hline 10 & 60 & 10 & 3.61 \\
\hline 11 & 45 & 10 & 4.01 \\
\hline 12 & 30 & 10 & $\underline{6.06}$ \\
\hline 13 & 15 & 10 & $\underline{13.76}$ \\
\hline 14 & 0 & 10 & INFINITE \\
\hline
\end{tabular}

As the elevation angle approaches zero degrees (the signal is arriving directly orthogonal to the array) the error is infinite. From these simulations it can be seen that the resulting DOA estimate is quite robust to phase error. It is interesting to note that a statistical standard deviation in phase error produces a DOA estimation error with lower standard deviation. At a $60^{\circ}$ elevation angle, 
for example, each of the antennas is given a standard deviation of $10^{\circ}$ phase error, yet the DOA estimation error has a resulting standard deviation of only $3.61^{\circ}$. Note that while the two element interferometer has accuracy that varies with azimuth angle; the three element interferometer has accuracy that remains stable as the azimuth angle is changed. This is intuitive since the geometry of the three element interferometer ensures that the three pairs of antennas are never simultaneously viewing the signal at an undesirable azimuth angle.

\subsection{Conclusion}

DOA estimation is a well known localization technique that could be applied to RFID tags. A phase comparison technique with a simple interferometer antenna array is a viable solution, and the configuration chosen in this section is a three element equilateral triangle with $16 \mathrm{~cm}$ spacing. An analysis of the proposed interferometer including the effect of antenna phase error on overall DOA estimation has been presented. 


\section{Chapter 4}

\section{Microstrip Patch Antenna Array}

\subsection{Introduction}

The microstrip patch antenna is the traditional RFID reader antenna. Patch antennas consist of a conductive patch and ground plane that are separated by a substrate material. Two commercial UHF RFID patch antennas are shown below:

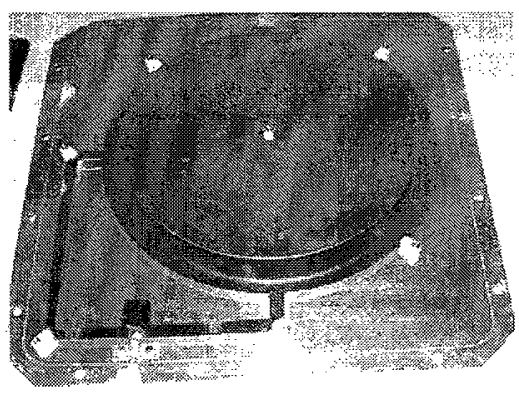

Figure 4.1: Impinj Far Field RFID IPJ-A1000 Antenna - Radome (Left) Radome Removed (Right)
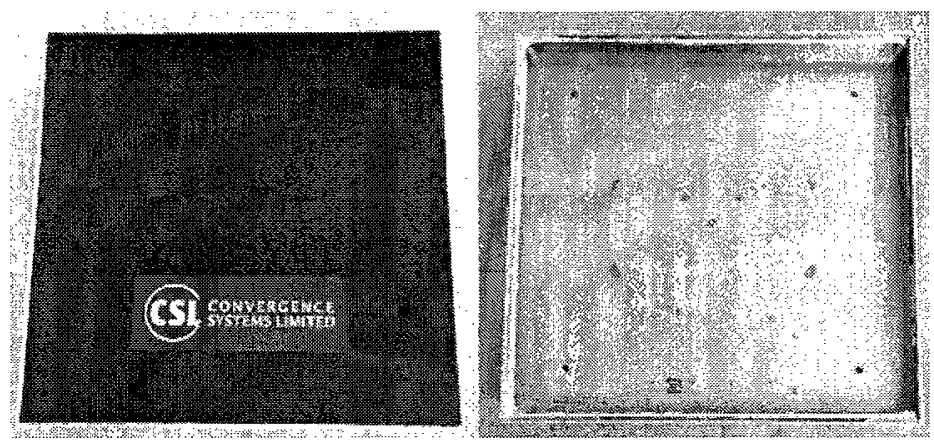

Figure 4.2: Convergence Systems Ltd. UHF RFID Patch Antenna - Radome (Left) Radome Removed (Right)

The two antenna designs are similar but there are differences in the feed designs and the Impinj antenna makes use of a stacked patch configuration to increase bandwidth. Both antennas have 
the patch elements suspended above the ground plane using an air substrate which increases gain and bandwidth. The downfall of using an air substrate is that the patch antennas are then too large to be spaced at half a wavelength (as is required for the chosen interferometer configuration). By using a substrate material with a higher permittivity, a size reduced patch antenna can be developed and used for this application.

In this chapter, the basic theory of a circular microstrip patch antenna is given. The design of a patch antenna for an RFID interferometer is described, and the construction and testing of the individual antenna and array are presented.

\subsection{Circular Patch Antenna Theory - Cavity Model}

Circular patch antenna geometry was chosen for the design, for aesthetic reasons. The gain of the circular patch was found to be a few tenths of a $\mathrm{dB}$ higher from simulation. The antenna theory in this section is focused on circular patch geometry.

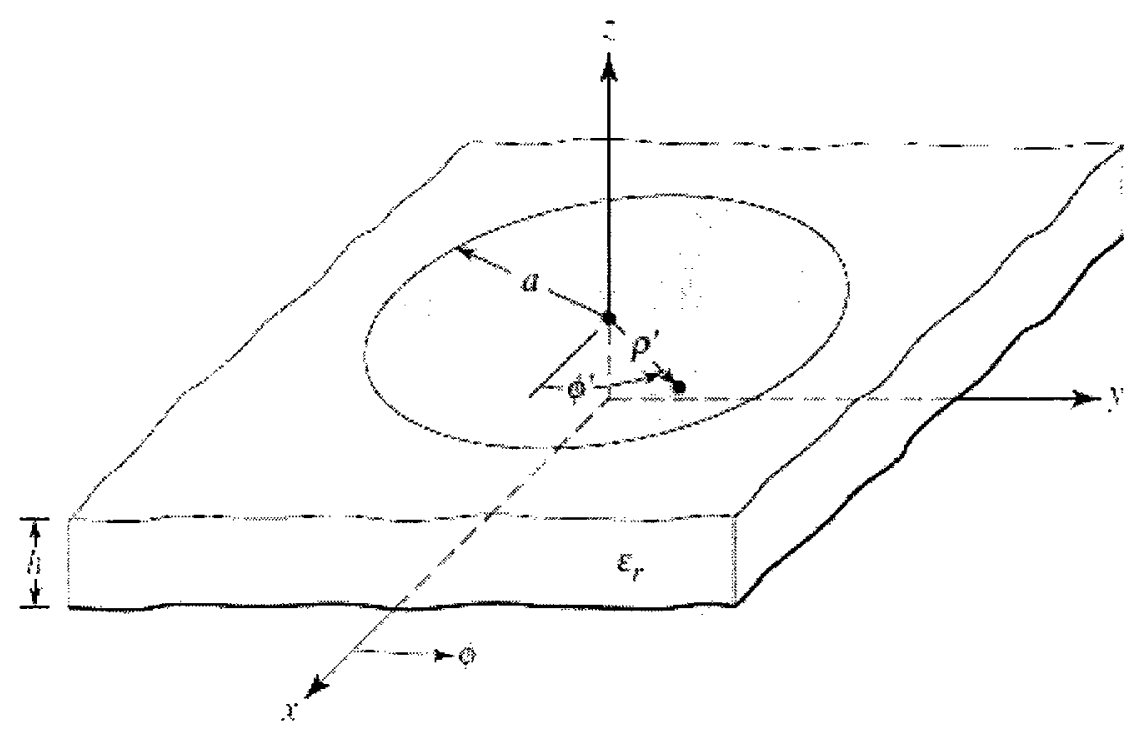

Figure 4.3: Geometry of circular microstrip patch antenna [11] 
The circular conducting patch of radius $a$ is separated from a ground plane by a substrate material of height $h$ as depicted in Figure 4.3. There are several methods available to analyze a microstrip patch antennas including the cavity model, full wave analysis, generalized transmission-line model, and method of moments. Microstrip antenna theory provided in this section is from numerous books [11], [12],[13]. The cavity model will be discussed here, because it provides accurate analysis and a good understanding of the circular patch.

When the height of the substrate is much less than a wavelength, the patch can be approximated as having an electric field with normal components (vertical between the ground plane and patch) and a magnetic field with transverse components. For small substrate heights there are only $\mathrm{TM}_{\mathrm{mn}}$ modes propagating in this structure. These approximations lead to a simplified cavity model of the patch antenna having two perfect electric conductors at the top and bottom to represent the conducting patch and ground plane. In the cavity model, the dielectric material does not extend past the edges of the patch. The periphery wall around the patch is modeled as a perfect magnetic conductor. A fictitious equivalent magnetic current (shown in Figure 4.4) is used in this model and by use of the equivalence principle the far-zone electric fields can be attained. 


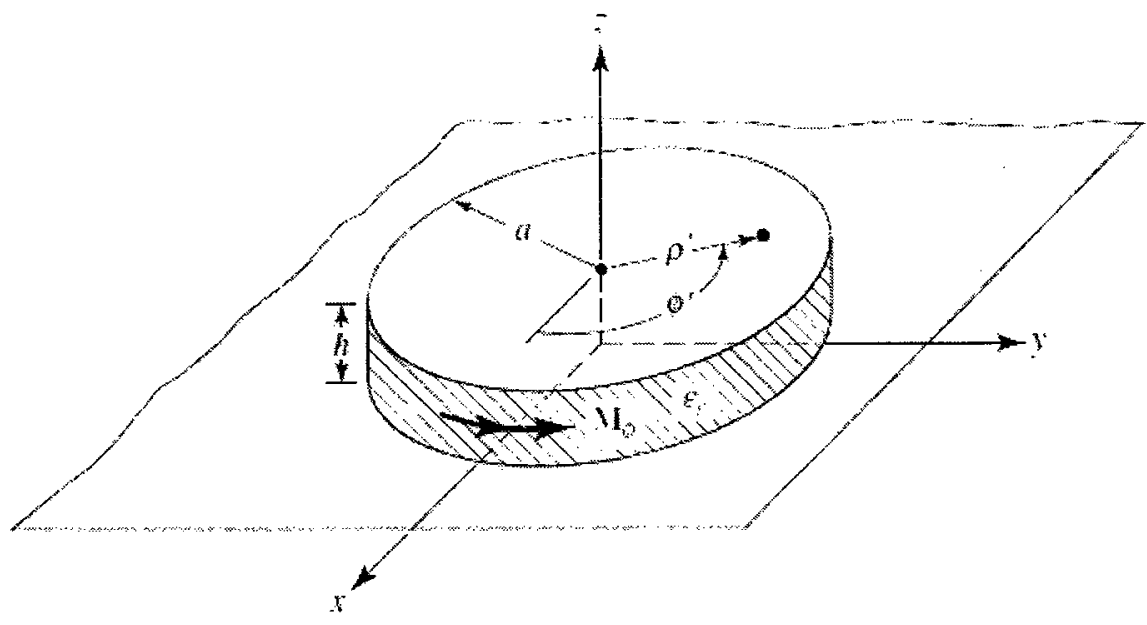

Figure 4.4:Cavity Model and Equivalent Magnetic Current Density for Circular Microstrip Patch Antenna [11]

In Figure 4.5, the field patterns and electric current are depicted for the circular patch (dominant $\mathrm{TM}_{11}$ mode). The normal components of the electric field and the traverse magnetic field are illustrated.



Figure 4.5: Electric and Magnetic Field Pattern of a Circular Microstrip Antenna at Resonance in the $T M_{11}$ mode [12] 


\subsubsection{Resonant Frequency}

The electric field inside the cavity is described by the following equations:

$$
\begin{gathered}
E_{z}=E_{0} J_{n}(k \rho) \cos (n \phi), \quad k=2 \pi \frac{\sqrt{\epsilon_{r}}}{\lambda_{0}} \\
E_{\rho}=0 \\
E_{\phi}=0
\end{gathered}
$$

Where $\mathrm{k}$ is the propagation constant, $\lambda_{0}$ is the wavelength in free space, and $\epsilon_{r}$ is the dielectric constant. $E_{0}$ is magnitude of the electric field, , $J_{n}(k \rho)$ are the Bessel functions of order $\mathrm{n}$. The magnetic field components are given below:

$$
\begin{gathered}
H_{r}=-\frac{j \omega \epsilon n}{k^{2} r} E_{0} J_{n}(k \rho) \sin (i n \phi) \\
H_{\phi}=-\frac{j \omega \epsilon}{k} E_{0} J_{n}^{\prime}(k \rho) \cos (i n \phi) \\
H_{z}=0
\end{gathered}
$$

Where $J_{n}^{\prime}$ is the derivative of the Bessel function with respect to its argument. $\omega$ is the angular frequency $(\omega=2 \pi f)$ and $\epsilon$ is the dielectric constant $\epsilon=\epsilon_{0} \epsilon_{r}$.

It is required that $J_{n}^{\prime}(k a)=0$, because of the open circuit condition that exists at the edge of the patch. For each mode there is an associated radius that is dependent on the zeros of the derivative of this Bessel function. The resonant frequency of each mode is given by Equation 4.7.

$$
f_{n m}=\frac{\chi^{\prime}{ }_{n m} * c}{2 \pi a_{e f f} \sqrt{\left(\epsilon_{r}\right)}}
$$

Where $\mathrm{c}$ is the speed of light and $a_{e f f}$ is the effective radius of the patch ( $a_{e f f}$ is slightly larger than the actual patch radius $a$ due to the fringing fields that occur at the edge of the patch). $\chi^{\prime}{ }_{n m}$ represents the zeros of $J_{m}^{\prime}(x)$, which determines the order of the resonant frequencies. For the 
dominant $\mathrm{TM}_{11}$ mode $\chi^{\prime}{ }_{11}$ is equal to 1.8412 giving the following expression for the resonant frequency.

$$
f_{r_{11}}=\frac{1.8412 * c}{2 \pi a_{e f f} \sqrt{\epsilon_{r}}}
$$

The approximate empirical relationship (Equation 4.9) has been developed to correctly size the radius of the patch for operation in the dominant $\mathrm{TM}_{11}$ mode.

$$
a_{e f f}=\frac{F}{\left\{1+\frac{2 h}{\pi \epsilon_{r} F}\left[\ln \left(\frac{\pi F}{2 h}\right)+1.7726\right]\right\}^{\frac{1}{2}}}, \quad F=8.791 * \frac{10^{9}}{f_{r} \sqrt{\epsilon_{r}}}
$$

In Equation 4.9 above the substrate height $h$ must be input in $\mathrm{cm}$.

\subsubsection{Fields Radiated}

In this cavity model the perfect magnetic walls around the periphery of the patch carry an equivalent magnetic current density $\mathrm{M}_{\mathrm{S}}$ (depicted in Figure 4.4). The presence of the ground plane can be taken into account using image theory which doubles the magnetic current density. From this fictitious magnetic current the simplified fields radiated from the patch can be found (explained step by step in [11]) and written as:

$$
\begin{aligned}
& E-P \text { lane }\left(\phi=0^{\circ}, 180^{\circ}, 0^{\circ} \leq \theta \leq 90^{\circ}\right) \\
& E_{\theta}=\frac{j k_{0} a_{e f f} V_{0} e^{-j k_{0} r}}{2 r}\left[J_{02}^{\prime}\right] \\
& E_{\phi}=0 \\
& H-P \text { lane }\left(\phi=90^{\circ}, 270^{\circ}, 0^{\circ} \leq \theta \leq 90^{\circ}\right) \\
& E_{\theta}=0 \\
& E_{\phi}=\frac{j k_{0} a_{e f f} V_{0} e^{-j k_{0} r}}{2 r}\left[\cos \theta J_{02}\right]
\end{aligned}
$$

Where $V_{0}$ represents the voltage of the excitation signal. 
These far fields have been plotted in Figure 4.6 comparing the cavity model with a measured antenna as well as the fields generated from a method of moment's analysis.

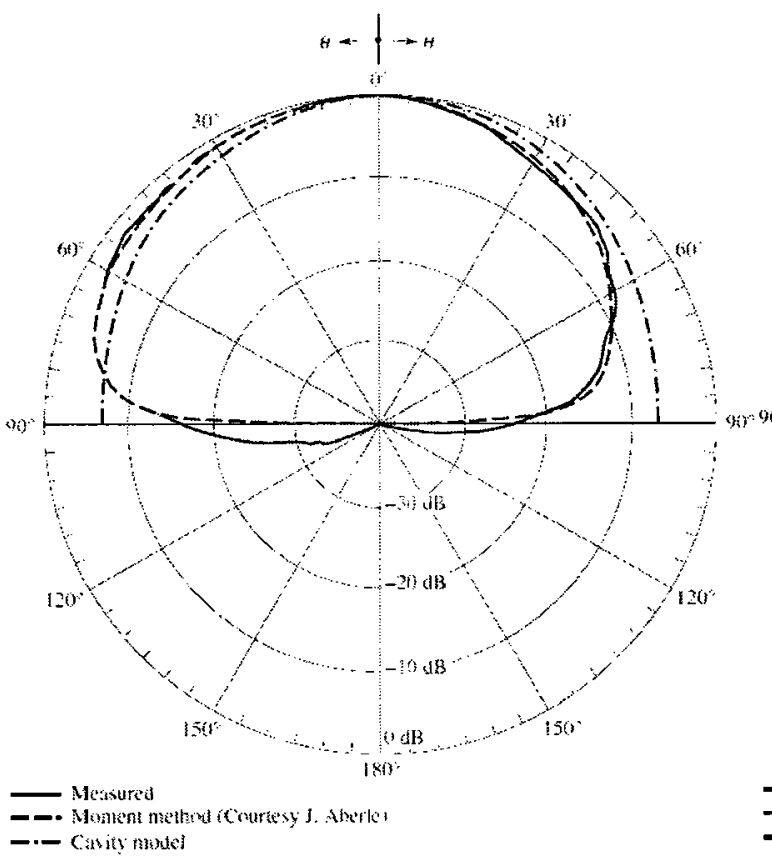

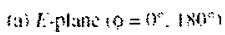

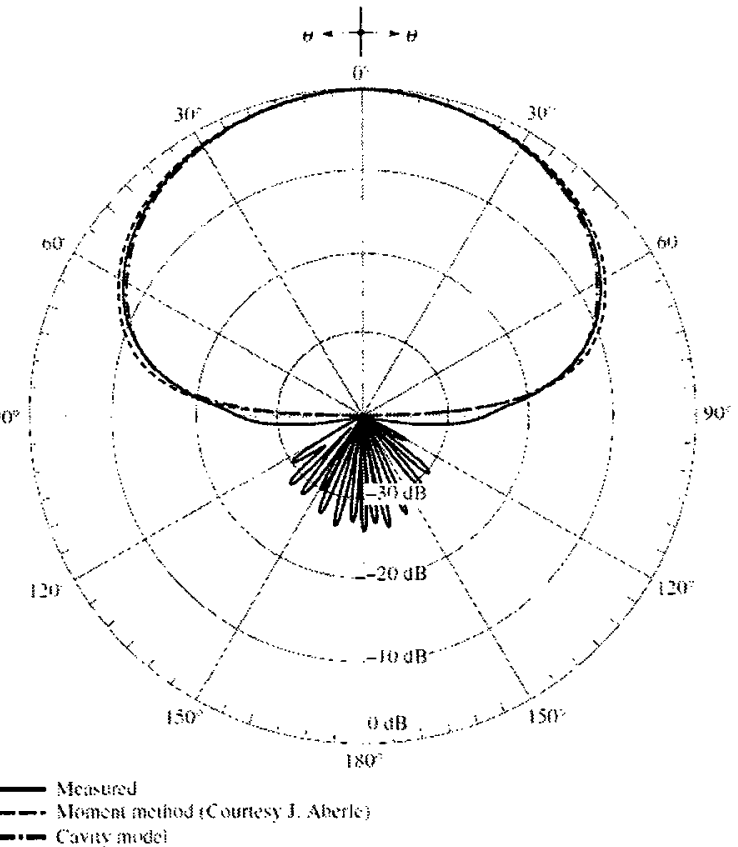

(n) $H$-plane $60=90.270^{n}$

Figure 4.6: Measured and Computed (based on moment method and cavity models) E- and H-plane patterns of circular microstrip patch antenna [11]

From the comparison in Figure 4.6, it can be seen that the cavity model provides accurate results for the radiation pattern provides accurate results when compared to the measurements.

\subsubsection{Input Impedance}

An antenna must efficiently radiate over a range of frequencies. In the case of RFID, this range is 902 to $928 \mathrm{MHz}$. Antennas are normally connected with a $50 \Omega$ transmission line and for maximum power transfer the of the antenna should be matched to $50 \Omega$. The type of feed and positioning of the feed is important for an impedance calculation. A patch antenna can be fed 
with a microstrip feed line, an aperture coupled feed, a proximity coupled feed line, or a probe feed. One of the most common techniques is the probe feed which can be seen in Figure 4.7.

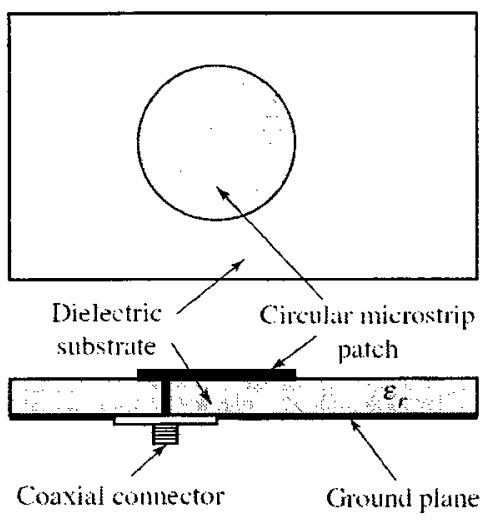

(b) Probe teed

\section{Figure 4.7: Probe Feed for Microstrip Patch Antenna [11]}

The probe feed consists of a coaxial connector with the inner probe directly attached to the microstrip patch as shown in Figure 4.7. The input impedance of the circular patch is difficult to analyze in closed form. The impedance seen is complex and both the resistive and reactive components vary with frequency. A typical plot is shown in Figure 4.8. 


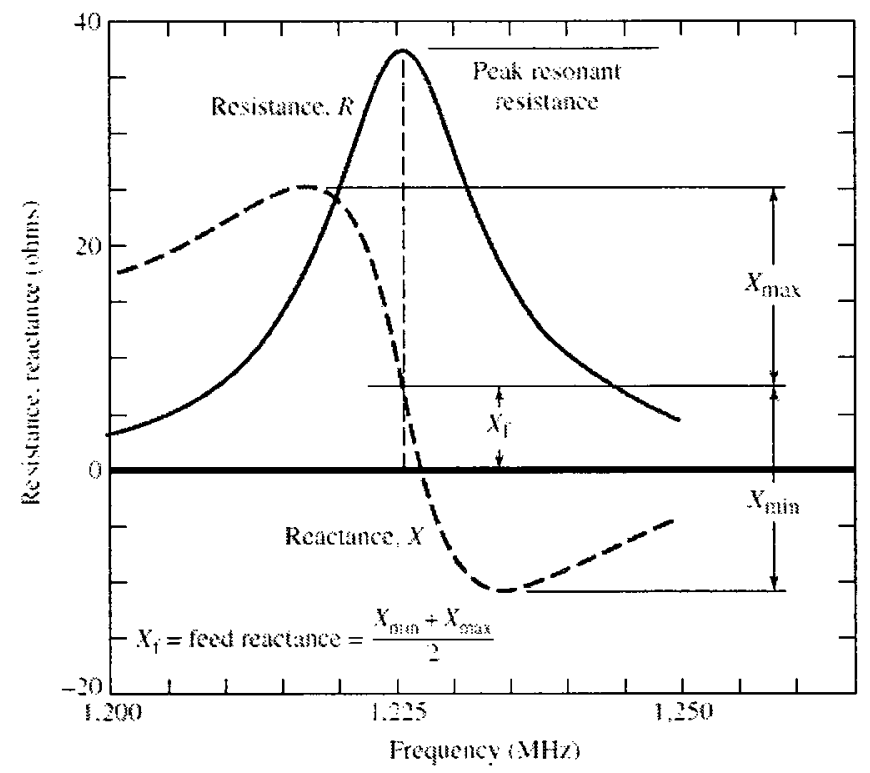

Figure 4.8: Typical Variation of Resistance and Reactance of Rectangular Microstrip Antenna versus Frequency[11]

Due to the large variation in input impedance, it is difficult to match the antenna over more than $3-5 \%$ bandwidth. The bandwidth can be increased by using a thicker substrate or a substrate with lower dielectric constant.

Not only does the input impedance vary with frequency, but the placement of the feed probe also changes the impedance. At the center of the patch the impedance is near zero and reaches a maximum at the edge. A common technique to match the patch is to correctly position the feed at a $50 \Omega$ point between the center and edge of the patch.

\subsubsection{Circular Polarization}

Circular polarization can be generated with a patch antenna by creating two orthogonal fields under the patch that have a $90^{\circ}$ phase difference with time. There are several methods of creating circular polarization. 


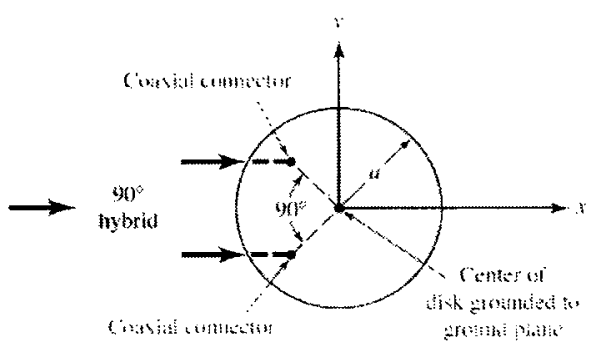

a)

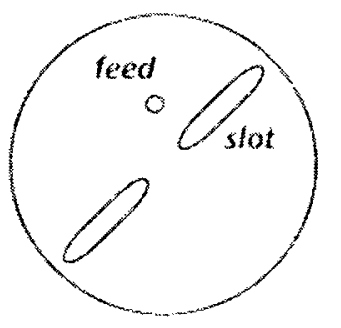

b)

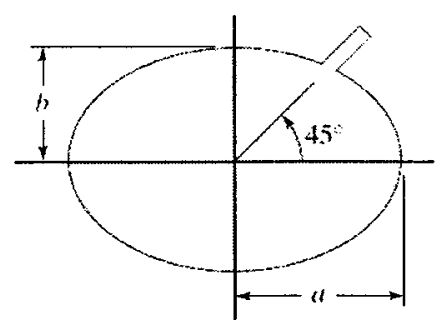

c)

Figure 4.9: Circular Polarization Techniques a) Dual Coaxial Feed [11], b) Single Feed with slots[1], c) Single Feed with Elliptical element and tabs[11].

Three of these methods are depicted in Figure 4.9: (a) a dual feed where one feed is given a $90^{\circ}$ phase shift, (b) a single feed that utilizes slots in the patch, (c) and a single feed using an elliptical element with tabs. While single feeds are easier to implement, a dual feed arrangement is capable of creating better polarization purity.

\subsection{Microstrip Patch Antenna Array Design}

A microstrip patch antenna array for operation in UHF RFID must meet many requirements, including bandwidth, polarization, cost and size limitations. Circular patch elements were chosen for this design and must physically fit in the configuration shown in Figure 4.10. 


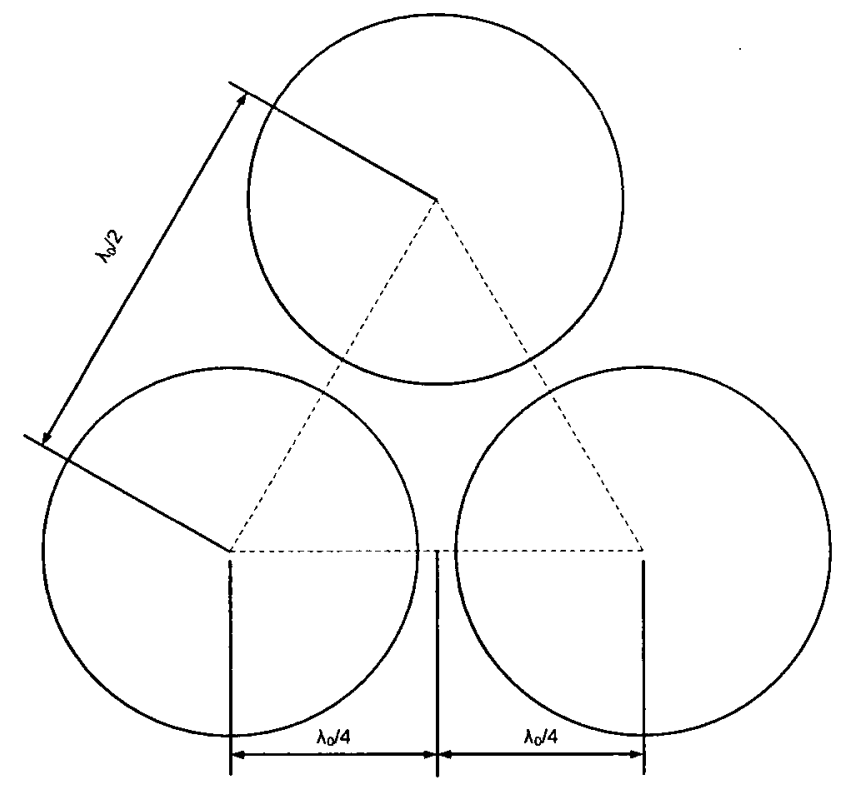

Figure 4.10: Interferometer Antenna Array Configuration

As discussed in the Chapter 3, the half wavelength spacing is $16 \mathrm{~cm}$. Therefore, the radius of the antennas must be less than $8 \mathrm{~cm}$, reserving room for the ground plane. The major design choices related to material, sizing and the feeding technique for this patch array are discussed below:

1. Substrate Material Selection: The size of a patch is proportional to $\lambda / \sqrt{\epsilon_{r}}$. It is common practice to reduce the size of a patch using a substrate material with a higher dielectric constant. Teflon was chosen for the substrate material because it is readily available and inexpensive compared to other microwave laminates with similar thickness. Teflon has an exceptionally low dissipation factor and its permittivity leads to a desirable patch size of $6.35 \mathrm{~cm}$.

2. Substrate Height: The substrate height of the antenna is a key parameter in controlling the operating bandwidth of the antenna. Computer simulations and experimental results verify that a Teflon substrate $0.9525 \mathrm{~cm}\left(3 / 8^{\prime \prime}\right)$ thick will provide sufficient bandwidth. 
3. Feeding Technique: The patch was chosen to be fed with a coaxial probe. This allows for simple prototyping because the input impedance can be easily adjusted by positioning the feed point. A dual feed is used in this design to create circular polarization. The signal at each feed is input in phase quadrature as shown in Figure 4.9 a). This dual feed technique is capable of producing better polarization purity over bandwidth than a single feed option.

4. Ground Plane: Patch antennas require a ground plane that is moderately larger than the patch itself. Increasing the ground plane reduces the amount of energy radiated behind the antenna. The ground plane must be a sufficient size for low back lobe radiation. An octagonal shaped ground plane was chosen because it is easier to cut than a circle and produces a more symmetrical azimuth radiation pattern than a square.

The radius of the patch for $915 \mathrm{MHz}$ resonance was estimated with Equation 4.7 and tuned by computer simulation. A model of the patch antenna was created in Ansoft HFSS and is shown in Figure 4.11. 

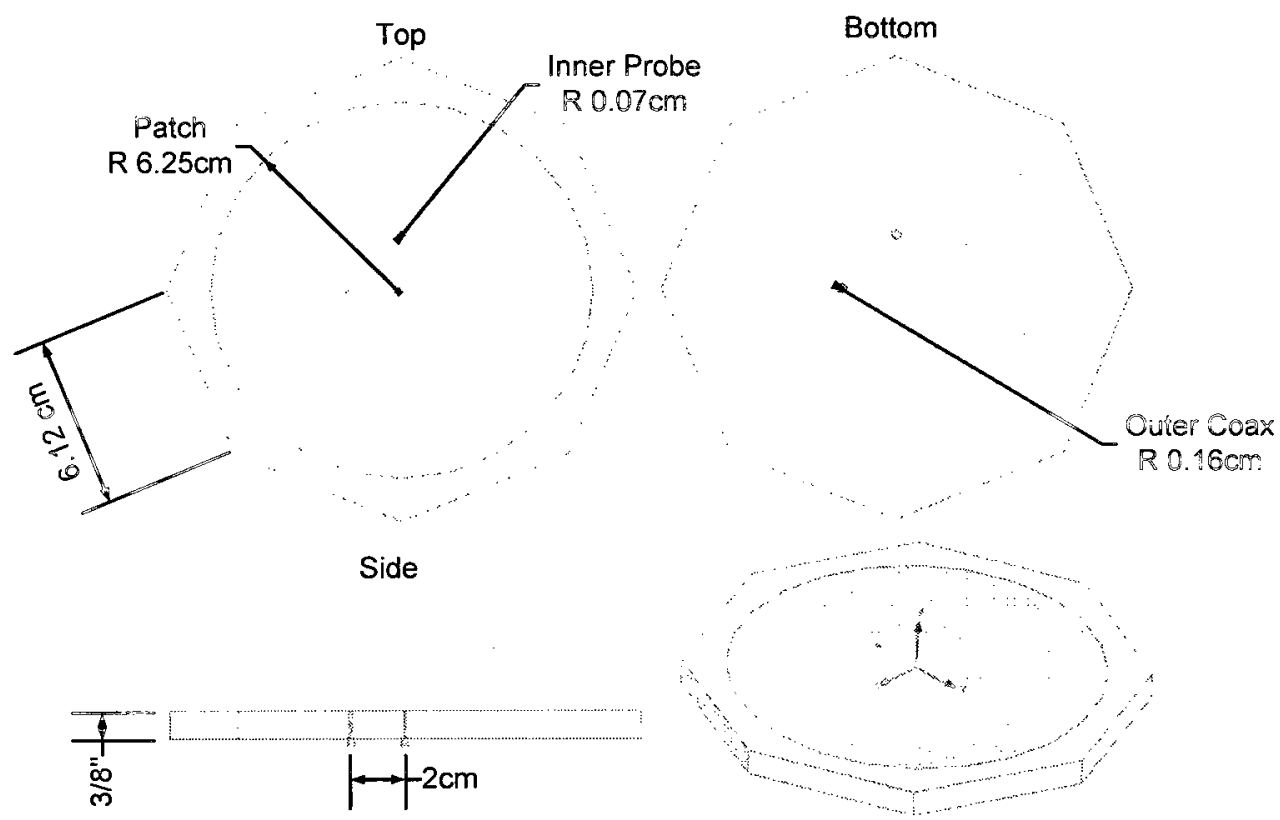

Figure 4.11: HFSS Model of Patch Antenna

The physical details of the patch antenna are described in Table 4.1.

Table 4.1: Patch Antenna, HFSS Model Parameters

\begin{tabular}{|l|l|}
\hline Patch Antenna Model Parameter & Value \\
\hline Substrate Material & Teflon \\
\hline Substrate Thickness & $0.9525 \mathrm{~cm}\left(3 / 8^{\prime \prime}\right)$ \\
\hline Patch Radius & $6.25 \mathrm{~cm}$ \\
\hline Side length of Substrate/Ground Octagon & $6.12 \mathrm{~cm}$ \\
\hline Probe Offset from center & $2 \mathrm{~cm}$ \\
\hline Inner Coaxial Probe Radius & $.07 \mathrm{~cm}$ \\
\hline Outer Coax Radius & $.16 \mathrm{~cm}$ \\
\hline
\end{tabular}


The position of the feed probe was found by observing the input impedance as the probe was moved from the edge of the patch to the center. Figure 4.12 depicts the change in impedance as the probe is moved from the edge of the patch to the center.

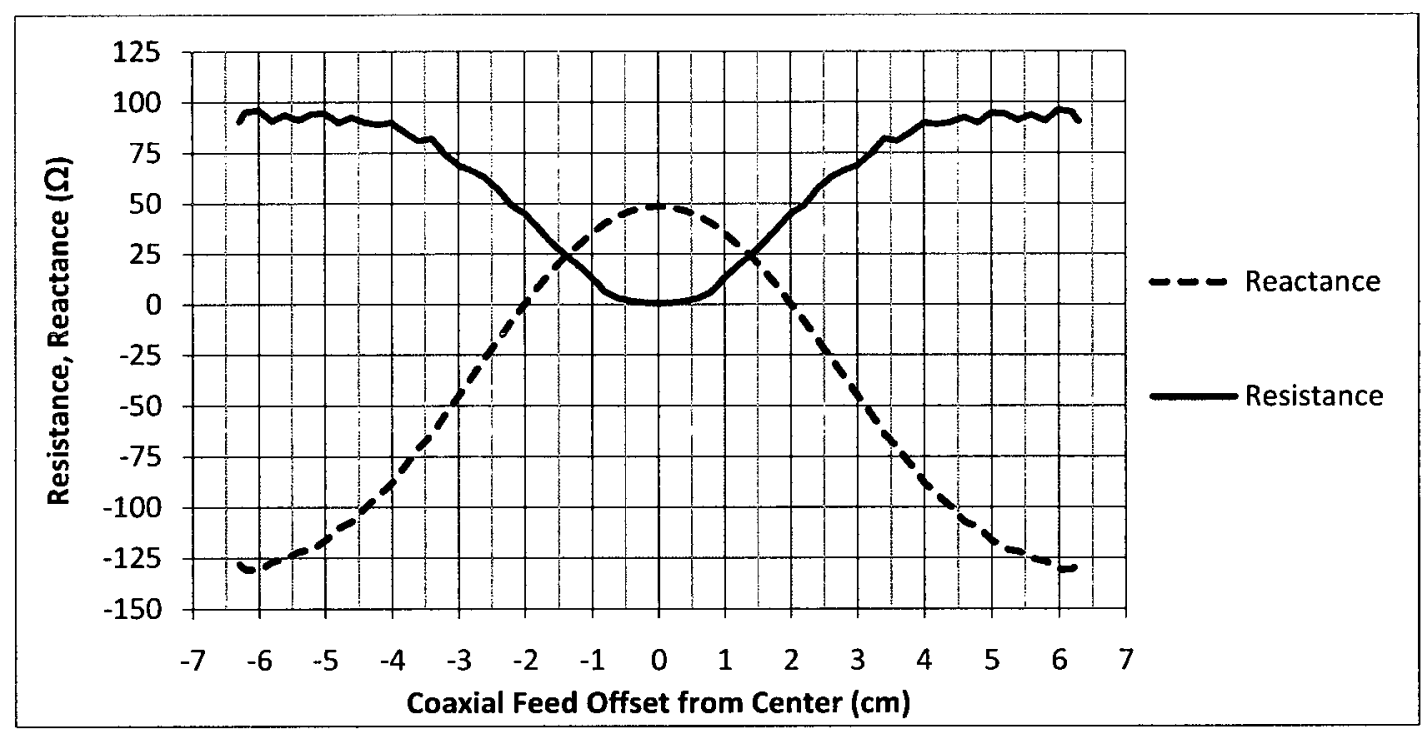

Figure 4.12: Patch Antenna Input Resistance and Reactance as a Function of Coaxial Probe Feed Offset from Center ( Microstrip Patch HFSS Simulation at 915 MHz)

The input resistance of the patch is approximately zero at the center of the patch and increases to about $100 \Omega$ at the edge. The reactance can also be seen to vary with feed position. In this case, a good $50 \Omega$ match is obtained by positioning the probe $2 \mathrm{~cm}$ offset from the center.

A plot of the input impedance as a function of frequency can be seen in Figure 4.13. 


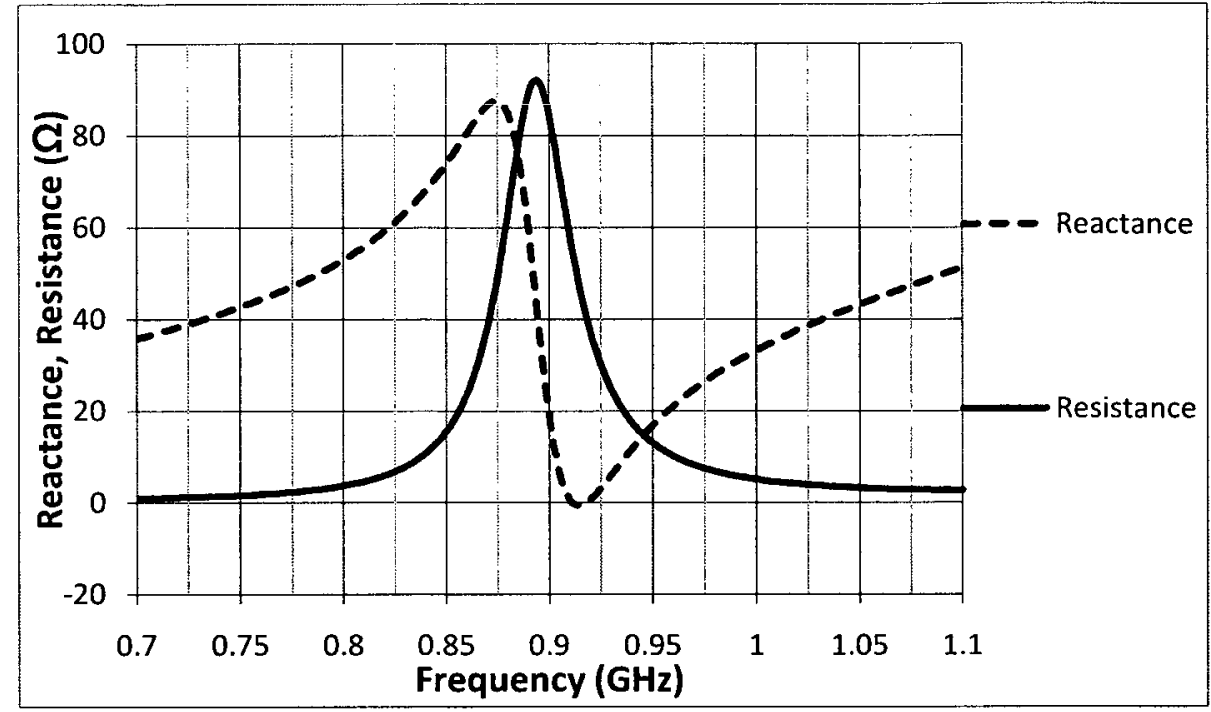

Figure 4.13: Patch Antenna Input Resistance and Reactance as a Function Frequency (HFSS Simulation)

Figure 4.13 shows an impedance plot as expected for a microstrip patch antenna. The positioning of the feeed achieves a near perfect $50 \Omega$ match at $915 \mathrm{MHz}$. The match and bandwidth of the antenna are better visualized by looking at the reflection coefficient in Figure 4.14. 


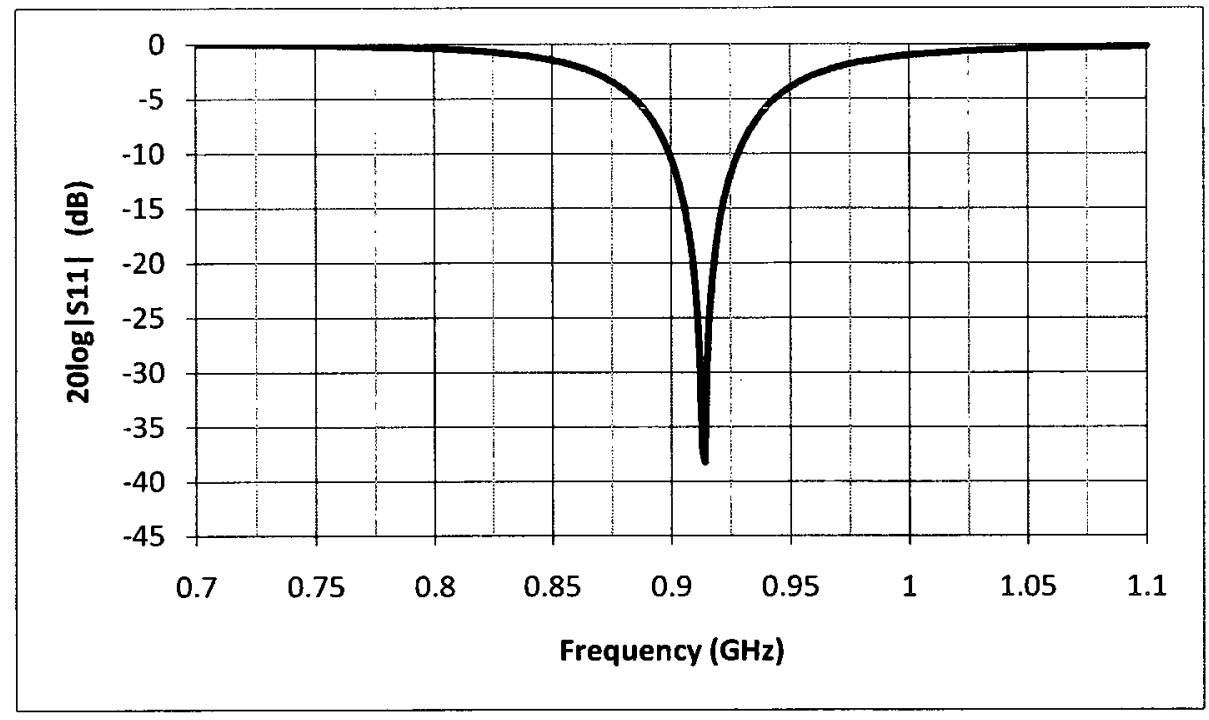

Figure 4.14: Simulated Return Loss (Microstrip Patch HFSS Simulation)

Figure 4.14 shows that the return loss is better than $10 \mathrm{~dB}$ from $902-928 \mathrm{MHz}$.

\subsection{Verification of Input Impedance}

A prototype antenna has been built to verify the feed offset location and the input impedance. The prototype patch antenna was built with a $0.9525 \mathrm{~cm} \mathrm{(3/8")} \mathrm{Teflon} \mathrm{sheet,} \mathrm{copper} \mathrm{tape,} \mathrm{a} \mathrm{PCB} \mathrm{feed}$ network and two SMA connectors. The prototype can be seen in Figure 4.15. 

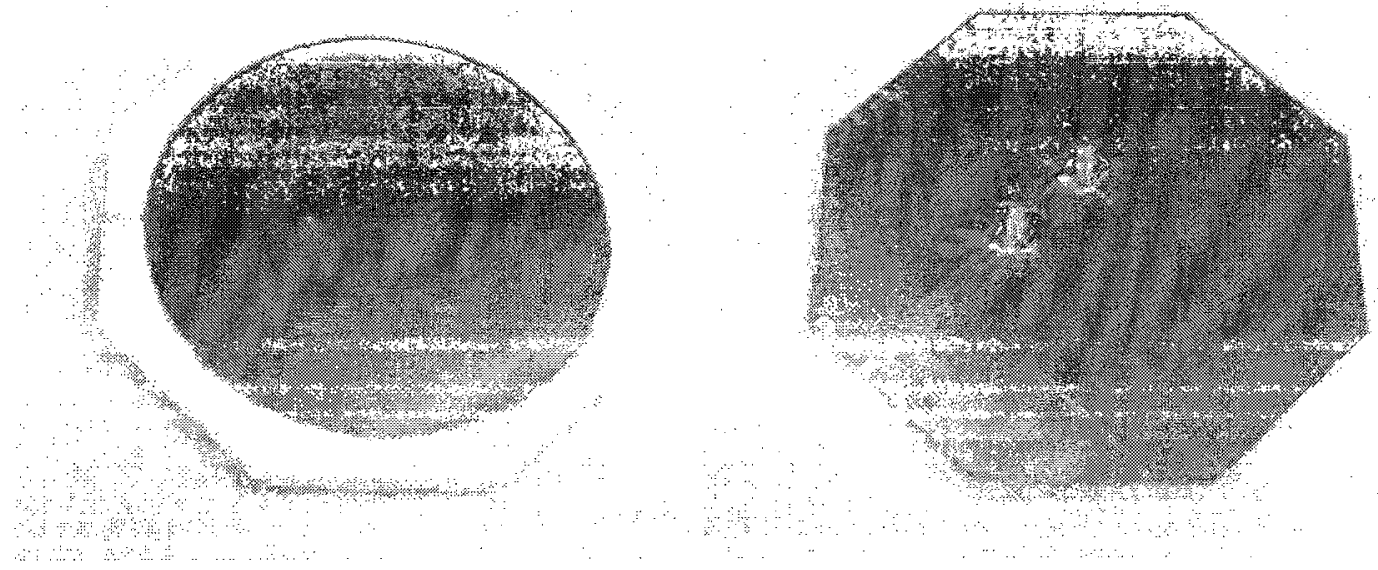

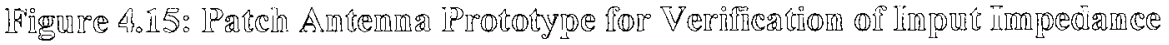

The S-parameters of the two port antenna were measured with a VNA and are compared with the simulated resulis in Figure 4.16.

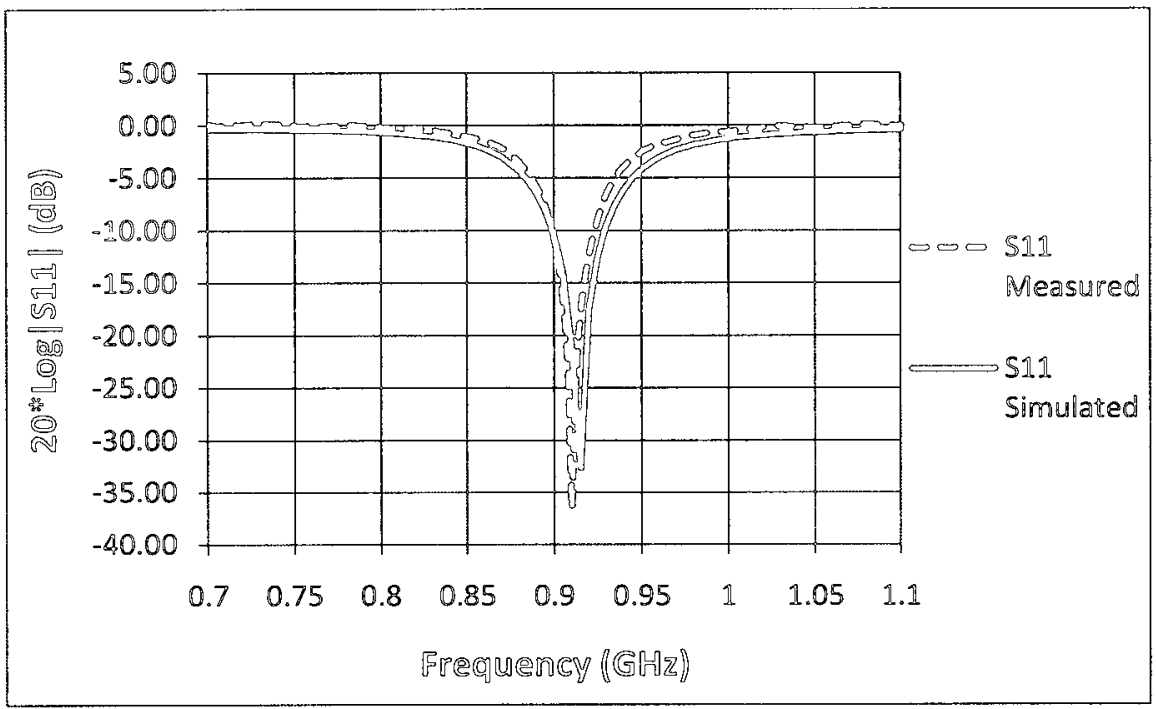

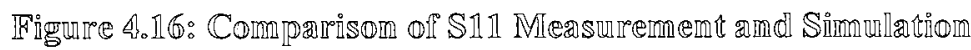

The simulated and measured retum loss curves in Figure 4.16 are nearly identical. 


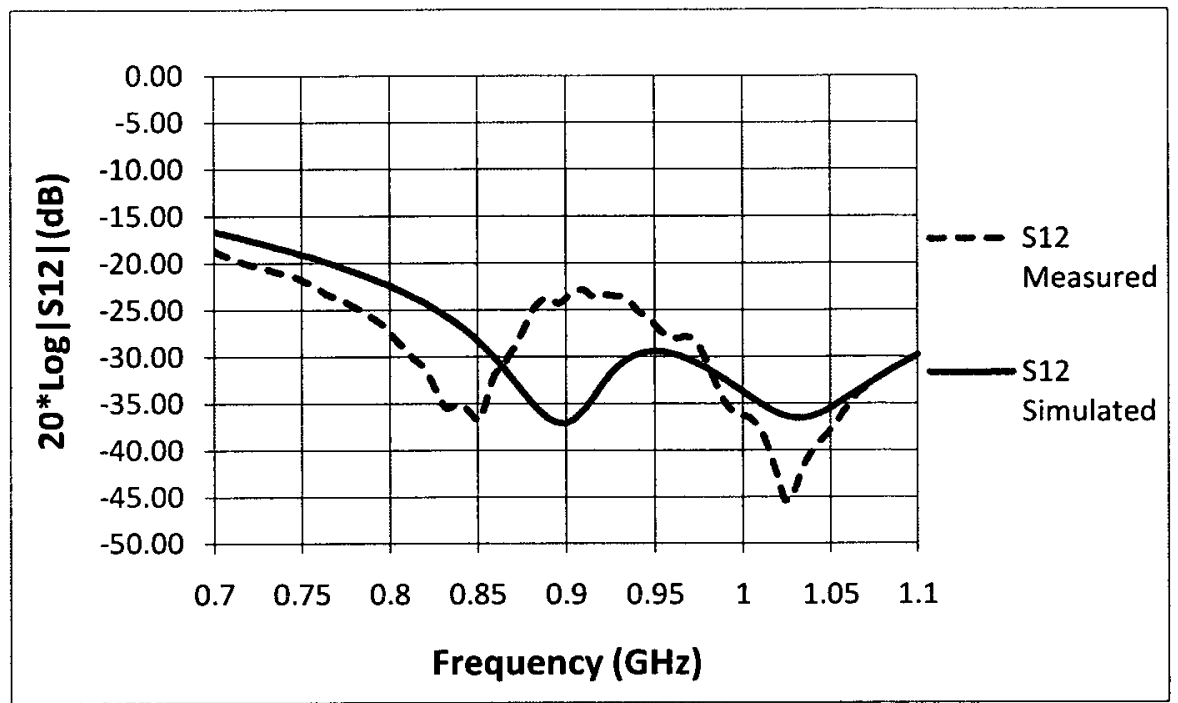

Figure 4.17: Comparison of S12 Measurement and Simulation

The plot of $\mathrm{S}_{12}$ in Figure 4.17 shows that the coupling between the two ports is less than $23 \mathrm{~dB}$ over the $902-928 \mathrm{MHz}$ band. The $\mathrm{S}_{12}$ simulation results are shifted if frequency when compared to the measurement. This is likely due to error tolerance when hand drilling the holes for the placement of the probes and cutting the patch. Overall the results validate the HFSS model and show that the feed offset provides a near perfect $50 \Omega$ match at $915 \mathrm{MHz}$.

\subsection{Feed Network Design}

The feed network was designed on a standard .062" FR4 board. To create circular polarization the two ports must be excited with equal amplitude and in phase quadrature. A Wilkinson power splitter was designed to equally divide the input signal. Detailed analysis of the Wilkinson power splitter can be found in D. Pozar's "Microwave Engineering" [14]. A delay line with $90^{\circ}$ electrical length was used on one of the Wilkinson outputs to provide the quadrature phasing. The model and PCB can be seen in Figure 4.18. 




Figure 4.18: PCB Feed network, Model (Left) Fabricated (Right)

The feed network was designed so that the patch would be fed at the correct feed positions with a $90^{\circ}$ phase difference between the two balanced signals. The measured phase is shown in Figure 4.19.

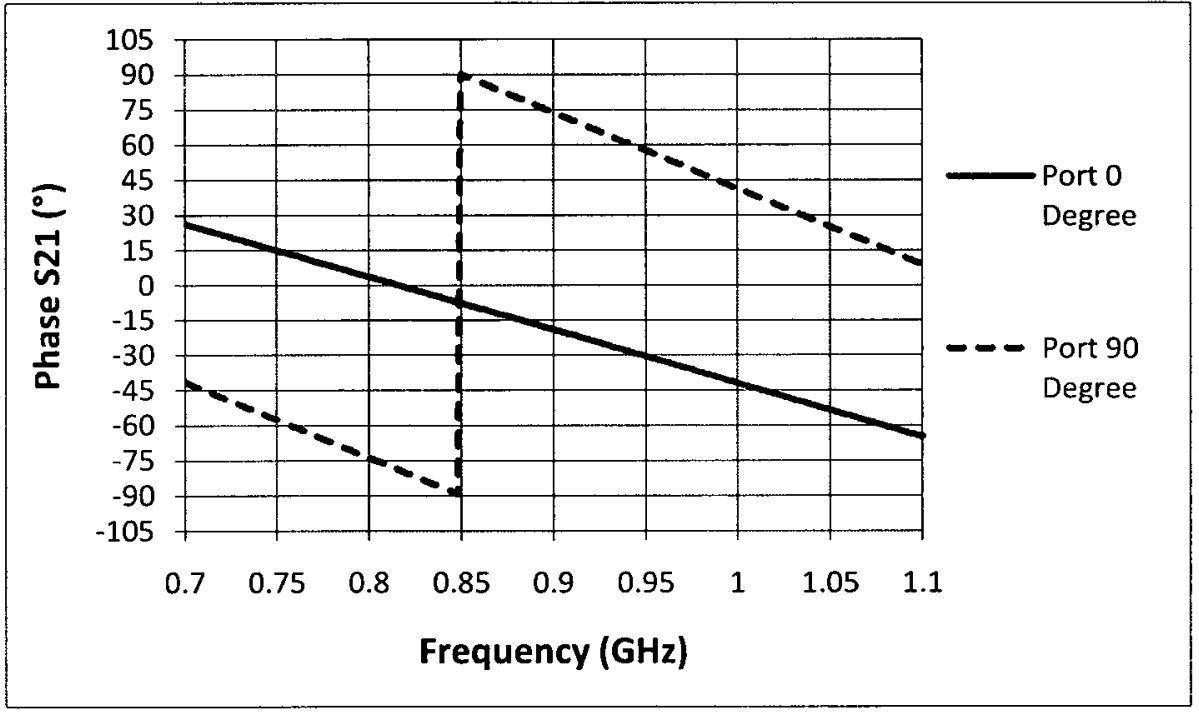

Figure 4.19: PCB Feed network Measured Phase versus Frequency 
The measured phase difference between the signals at $915 \mathrm{MHz}$ is $91.5^{\circ}$ and the worst case phase difference occurs at $902 \mathrm{MHz}$ with $92.6^{\circ}$ of phase difference.

The measured magnitude of the output signals from the feed network are shown in Figure 4.20.

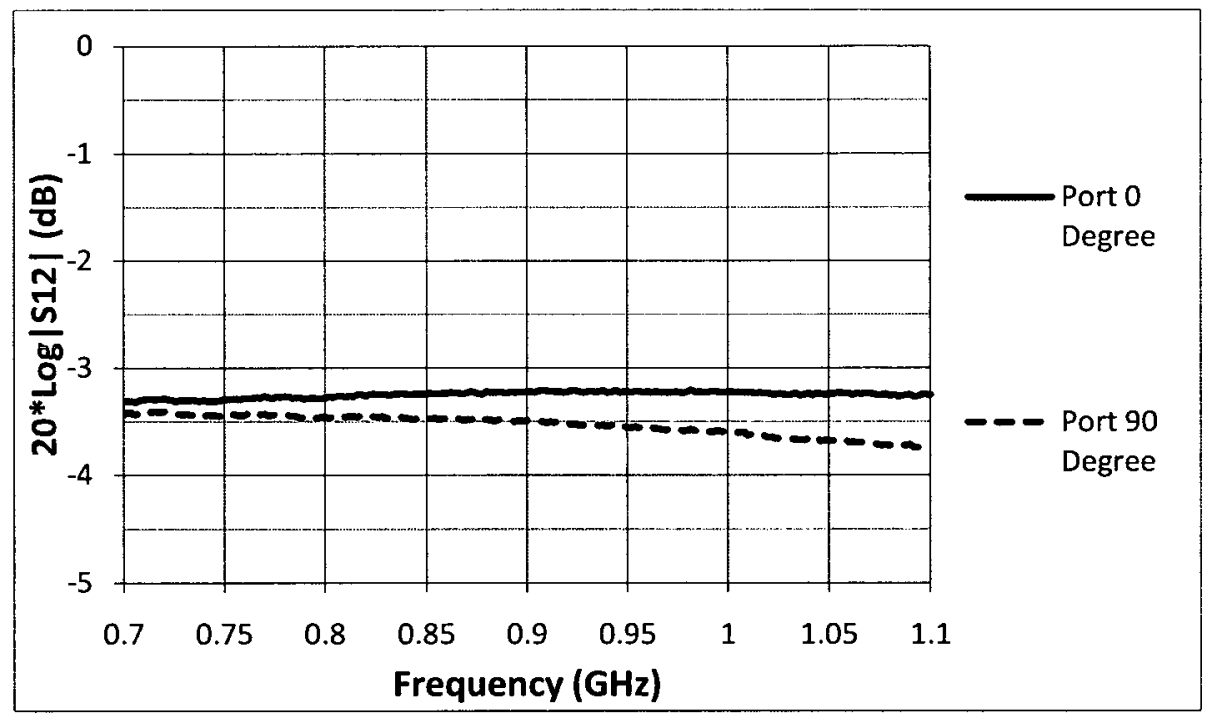

Figure 4.20: PCB Feed network Measured Amplitude of Output Signals

Ideally if the signals were equally split the power level at the output would be $-3 \mathrm{~dB}$. Due to losses in the feed network the amplitude is reduced which will lower the overall gain of the antenna. At $915 \mathrm{MHz}$ the output signal of the $0^{\circ}$ and $90^{\circ}$ ports are $-3.22 \mathrm{~dB}$ and $-3.51 \mathrm{~dB}$ respectively. The difference in amplitude at the ports will also decrease the polarization purity of the antenna. The performance could be improved by using a less lossy substrate.

\subsection{Microstrip Patch Antenna Fabrication and Testing}

The final patch antenna was created with a Teflon sheet affixed to the PCB feed network. The prototype antenna with the integrated PCB feed network can be seen in Figure 4.21. 


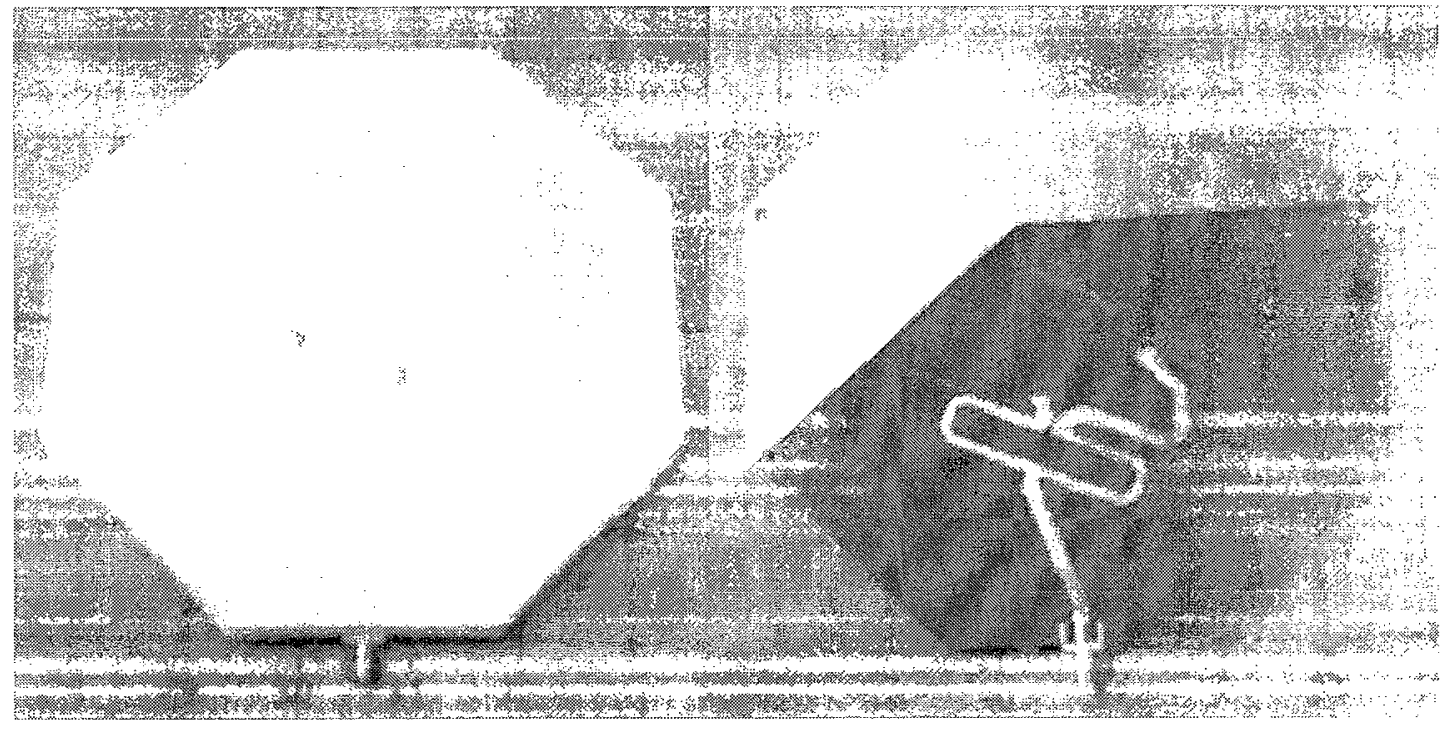

Figure 4.21: Patch Antenna with Feed Network, Top View (Left) Bottom View (Right)

Holes were drilled in the feed network and patch so that probes could be soldered in. Copper tape was used to create the patch. It was found that using the ground plane from the PCB was not adequate since air pockets were created between the Teflon and PCB that were not repeatable. Therefore copper tape was layered on the bottom side of the Teflon to create a consistent ground.

\subsubsection{Radiation Pattern}

The antenna has been measured in the anechoic chamber at the Communications Research Center (CRC) in Ottawa, Canada. A simulation of the antenna gain has also been completed. The simulation uses an idealized antenna without conductor, dielectric, or mismatch loss. A comparison between the measured and simulated radiation patterns can be seen in Figure 4.22, Figure 4.23, and Figure 4.24. 


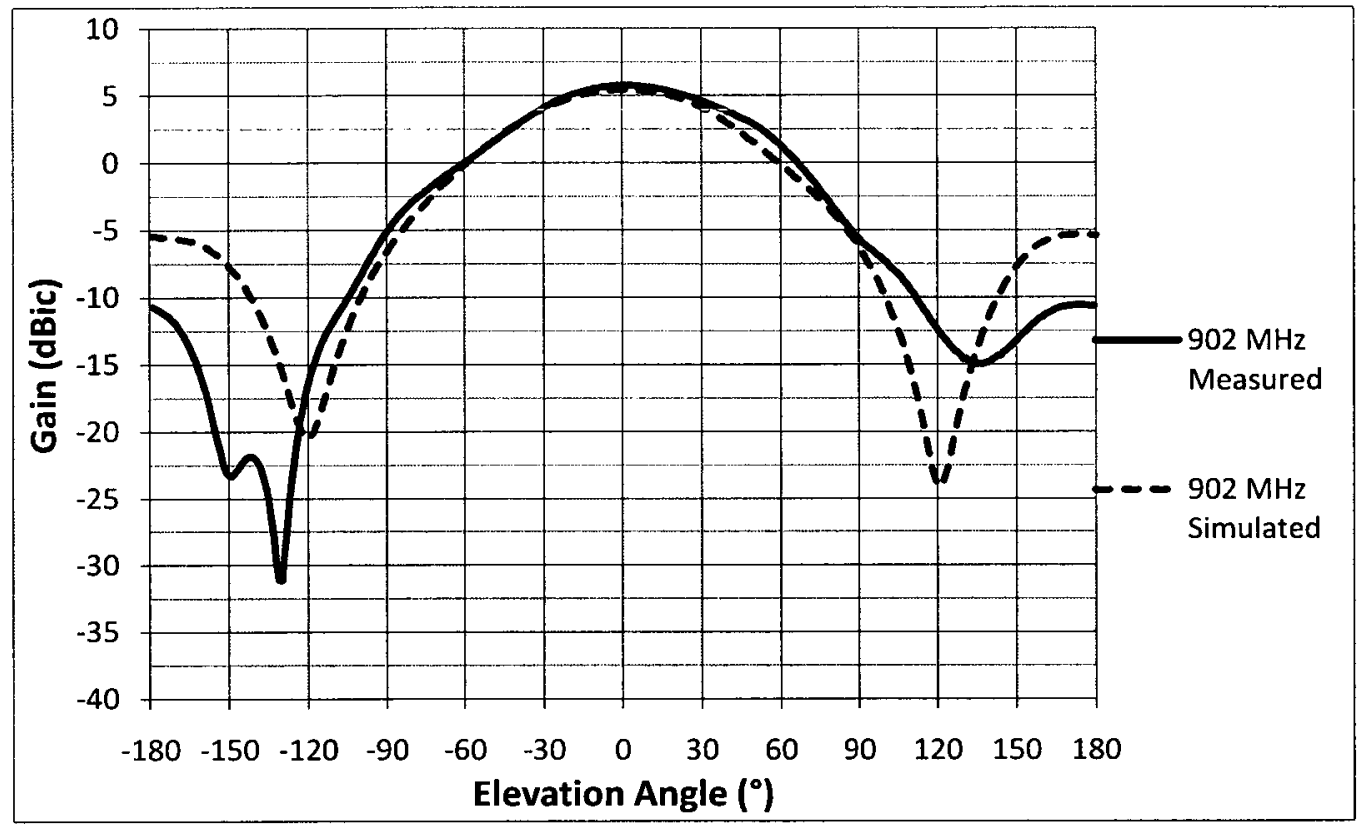

Figure 4.22: Elevation Pattern of Patch at $902 \mathrm{MHz}$

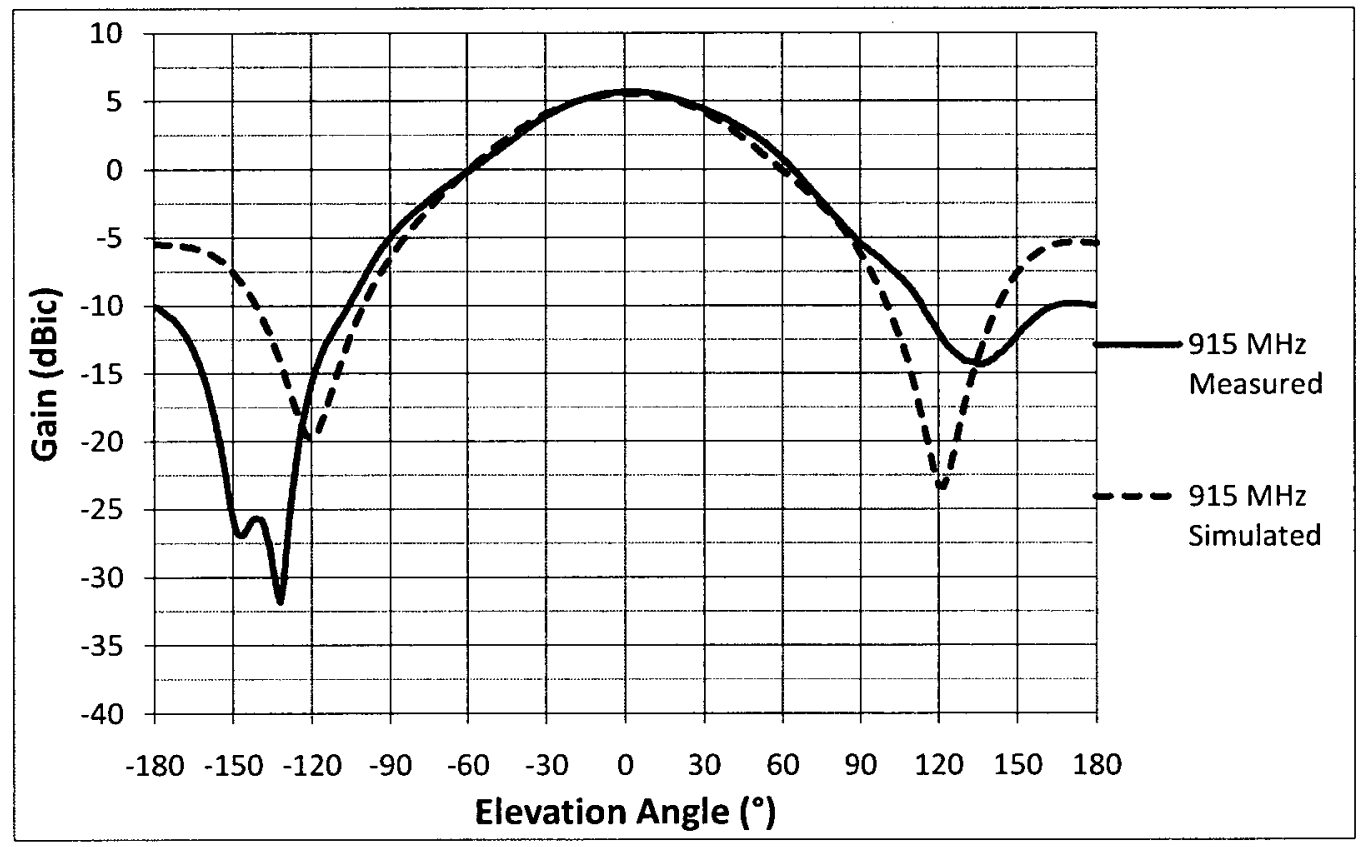

Figure 4.23: Elevation Pattern of Patch at $915 \mathrm{MHz}$ 


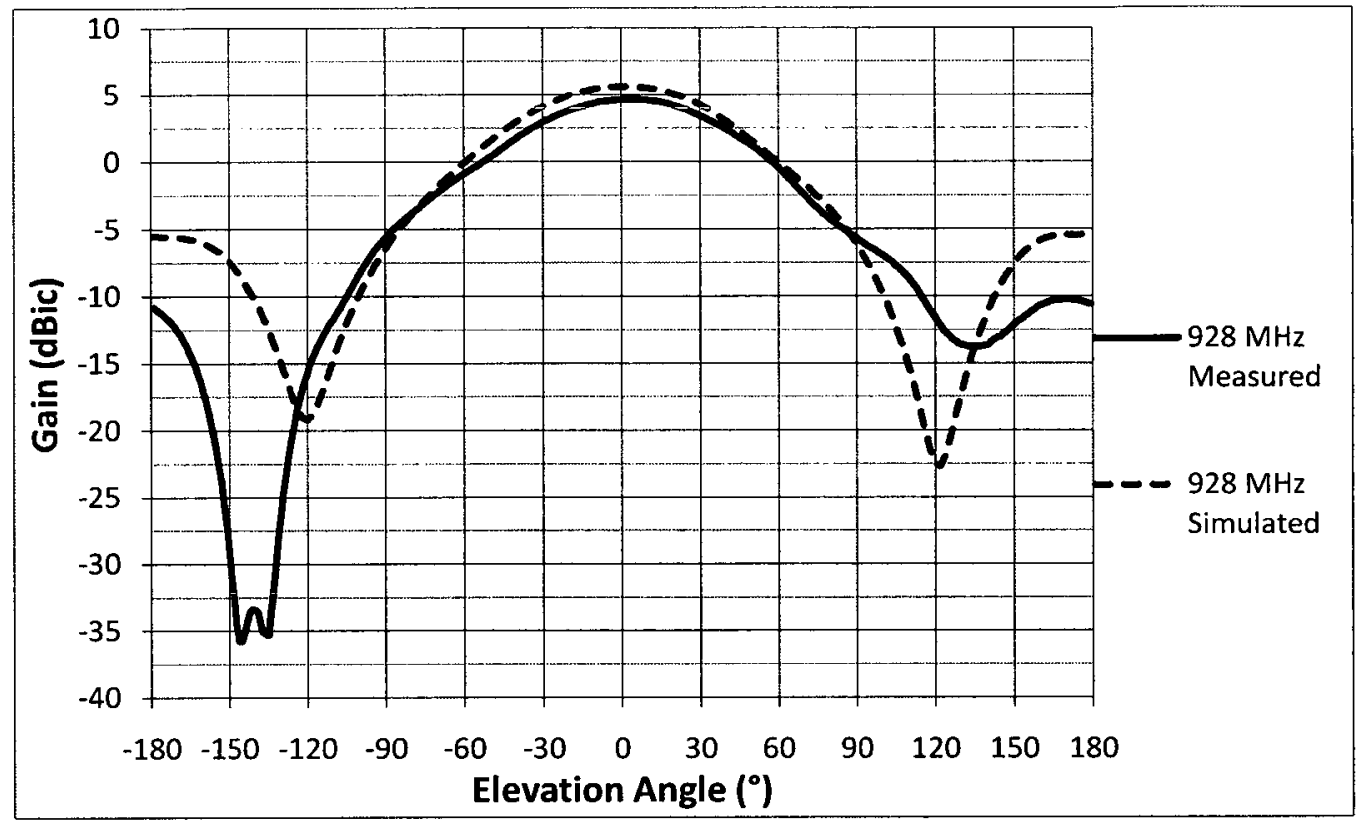

Figure 4.24: Elevation Pattern of Patch at $928 \mathrm{MHz}$

The pattern shapes match very well with the simulation results. The major difference is that the simulated pattern shows a larger back lobe (up to $5 \mathrm{~dB}$ larger). This discrepancy has likely been caused since a metal mount was used to attach the antenna when measuring its radiation pattern. The metal mount reduces the amount of radiation behind the antenna.

The peak measured gain of the antenna at $915 \mathrm{MHz}$ is $5.67 \mathrm{dBic}$. The peak gain of the simulation at $915 \mathrm{MHz}$ is $5.53 \mathrm{dBic}$. It is also believed that the reason the measured gain is slightly higher than the simulation is due to the metallic mount used in the measurement.

\subsubsection{Return Loss}

The return loss of the patch was found by measuring the S11 of the antenna with a HP8720-ES network analyzer. A plot of the return loss is shown in Figure 4.25. 


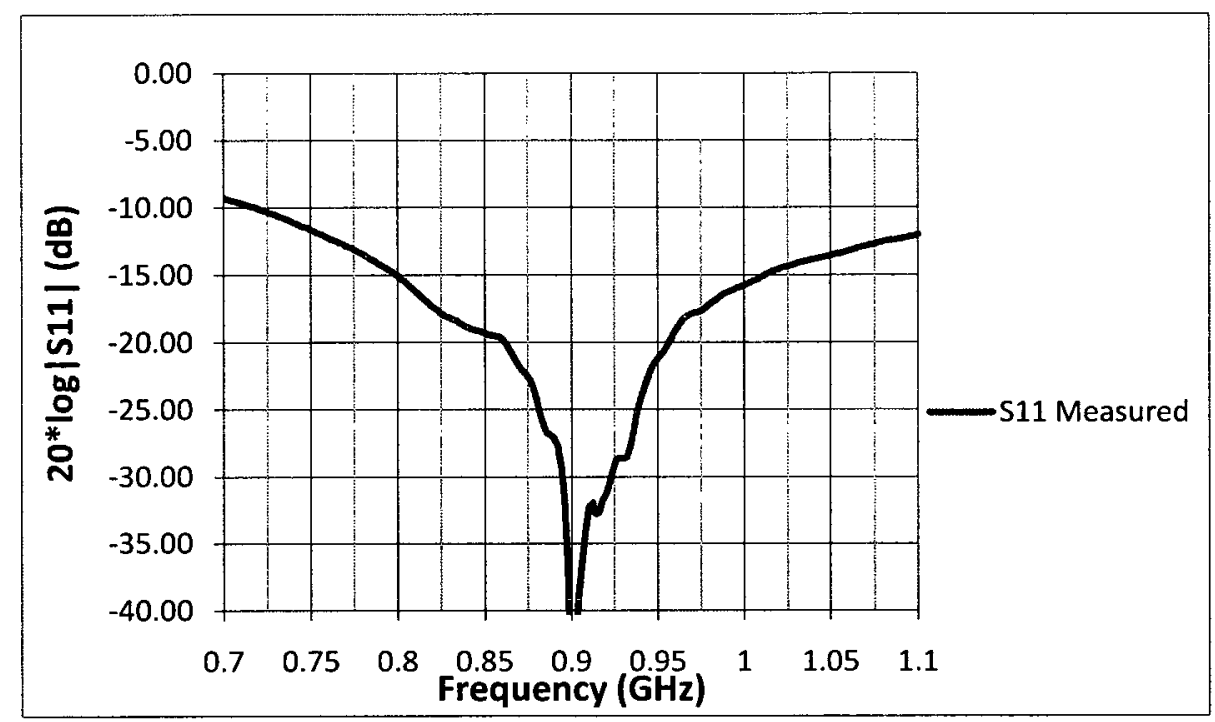

Figure 4.25: Measured Return Loss

The return loss of the antenna is excellent, better than $-25 \mathrm{~dB}$ over the $902-928 \mathrm{MHz}$ band.

Having a good match is especially important in RFID since the system often uses the antenna for mono-static (both transmit and receive) operation. RFID operates at the same frequency for transmit and receive, so there is no simple way to filter out the signal reflected from the antenna. A poor return loss can significantly degrade the sensitivity of the system[1].

\subsubsection{Phase and Gain versus Azimuth Angle}

It is important to have stable gain and phase versus azimuth angle when using an antenna in a direction finding interferometer array. The antenna was tested at $\mathrm{CRC}$ where the antenna was rotated in azimuth at different elevation cuts. The source antenna used in the test is linearly polarized. The diagram below describes the test configuration at an elevation cut of $15^{\circ}$. 

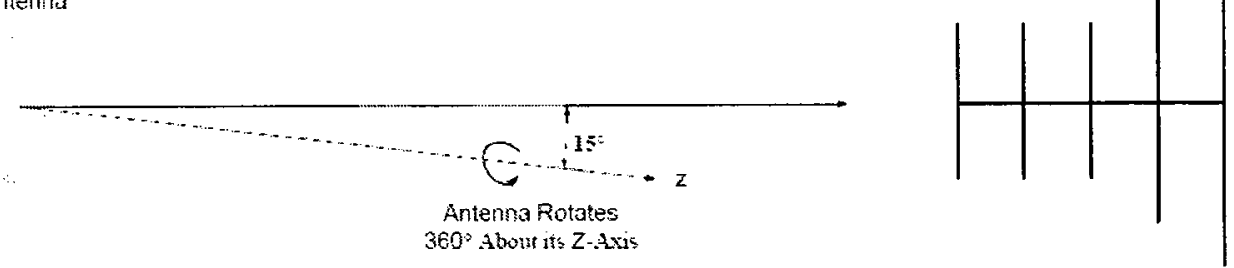

Figure 4.26: Phase versus Azimuth Angle Test Setup at $15^{\circ}$ Elevation Cut

An antenna with perfect circular polarization would have a linear phase change of $360^{\circ}$ as it is rotated a full turn azimuthally. The antenna was tested at incremental elevation cuts of $15^{\circ}$. The phase change at an elevation cut of $60^{\circ}$ can be seen in Figure 4.27.

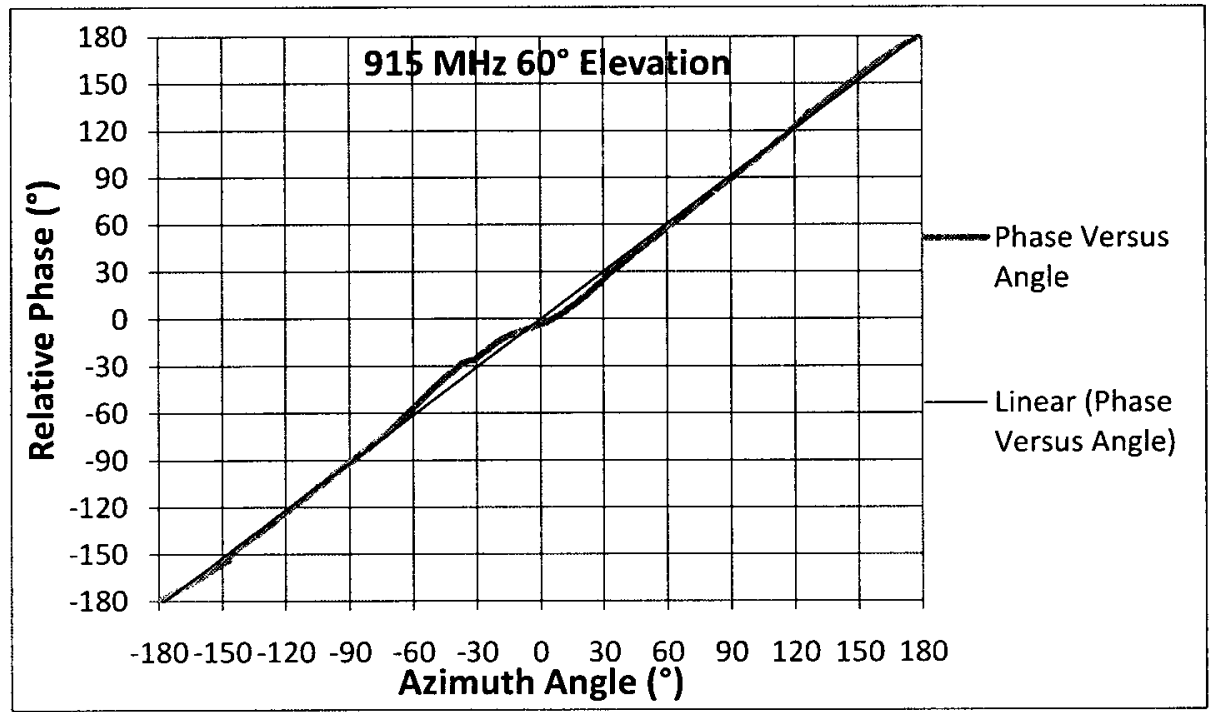

Figure 4.27: Patch Antenna Phase versus Azimuth Angle at $60^{\circ}$ Elevation Cut

The phase in Figure 4.27 changes very linearly over the 360 degree azimuth angle. The maximum deviation from the linear line is $7.1^{\circ}$ with a standard deviation of $3.6^{\circ}$.

Ideally a perfectly circularly polarized patch would have flat gain as it is rotated azimuthally and measured with a linear antenna. Since the patch has a finite axial ratio at different elevation 
angles, the magnitude of the gain will fluctuate as the antenna is rotated. Figure 4.28 shows the variation in gain with azimuth angle at a $60^{\circ}$ elevation cut.

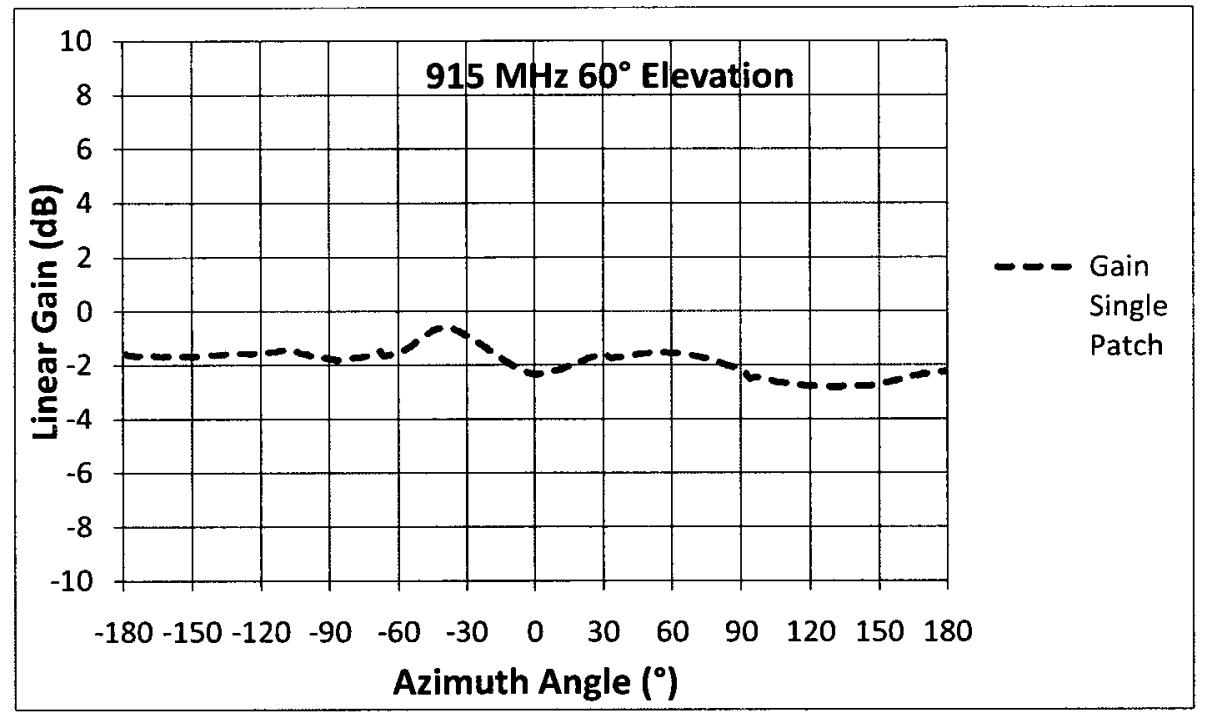

Figure 4.28: Gain versus Azimuth Angle at a $60^{\circ}$ Elevation Cut

By inspection of the plot, there is significant non-linear and non-symmetrical distortion. This can be attributed to the fact that the antenna is handmade with approximately one millimeter of tolerance, also antenna measurements suffer from distortion caused from the cables. The results from the elevation cut at $15^{\circ}$ intervals are tabulated in Table 4.2 below. 
Table 4.2: Azimuth Gain (Linear) and Phase Deviation of Single Patch Antenna

\begin{tabular}{|l|l||l|l|l|l|l|}
\hline $\begin{array}{l}\text { Elevation } \\
\text { Angle }\end{array}$ & $\begin{array}{l}\text { Average } \\
\text { Peak } \\
\text { Gain (dB) }\end{array}$ & $\begin{array}{l}\text { Maximum } \\
\text { Peak } \\
\text { Gain (dB) }\end{array}$ & $\begin{array}{l}\text { Minimum } \\
\text { Peak } \\
\text { Gain (dB) }\end{array}$ & $\begin{array}{l}\text { Gain } \boldsymbol{( d B )} \\
(\mathbf{d B})\end{array}$ & $\begin{array}{l}\text { Max Phase } \\
\text { Variance( }\end{array}$ & Phase $\boldsymbol{\sigma}\left(^{\circ}\right.$ ) \\
\hline $0^{\circ}$ (Nadir) & 3.1 & 3.4 & 2.6 & 0.3 & 4.3 & 2.3 \\
\hline $15^{\circ}$ & 2.6 & 3.2 & 2.1 & 0.3 & 3.4 & 2.0 \\
\hline $30^{\circ}$ & 1.7 & 2.3 & 1 & 0.3 & 3.7 & 1.8 \\
\hline $45^{\circ}$ & 0.2 & 0.6 & -0.1 & 0.2 & 5.4 & 2.1 \\
\hline $60^{\circ}$ & -1.9 & -0.6 & -2.8 & 0.5 & 7.1 & 3.6 \\
\hline $75^{\circ}$ & -5.2 & -3.9 & -8.4 & 1.1 & 10.3 & 5.3 \\
\hline $90^{\circ}$ & -9.1 & -7 & -15.7 & 2.4 & 19.2 & 8.6 \\
\hline
\end{tabular}

Table 4.2 gives a quick view of the deviation in gain and phase with azimuth angle. The axial ratio of the antenna can be found by taking the difference between the minimum and maximum gain. The axial ratio is better than $2.2 \mathrm{~dB}$ over the elevation angles $0^{\circ}-60^{\circ}$. The phase variation is also low $\left(\sigma<3.6^{\circ}\right)$ over the $0^{\circ}-60^{\circ}$ elevation angles but rapidly increases at steeper elevation angles. These results are as expected since the circular polarization of the patch is known to become more elliptical as elevation angles approach $90^{\circ}$.

\subsection{Microstrip Patch Interferometer Array}

Three of the fabricated patch antennas have been configured into the three element interferometer array proposed in chapter 4 . The purpose of this section is to investigate the interaction between the three antennas. It is possible that the elements will significantly distort the phase and gain patterns. The patch antenna interferometer array can be seen in Figure 4.29. 


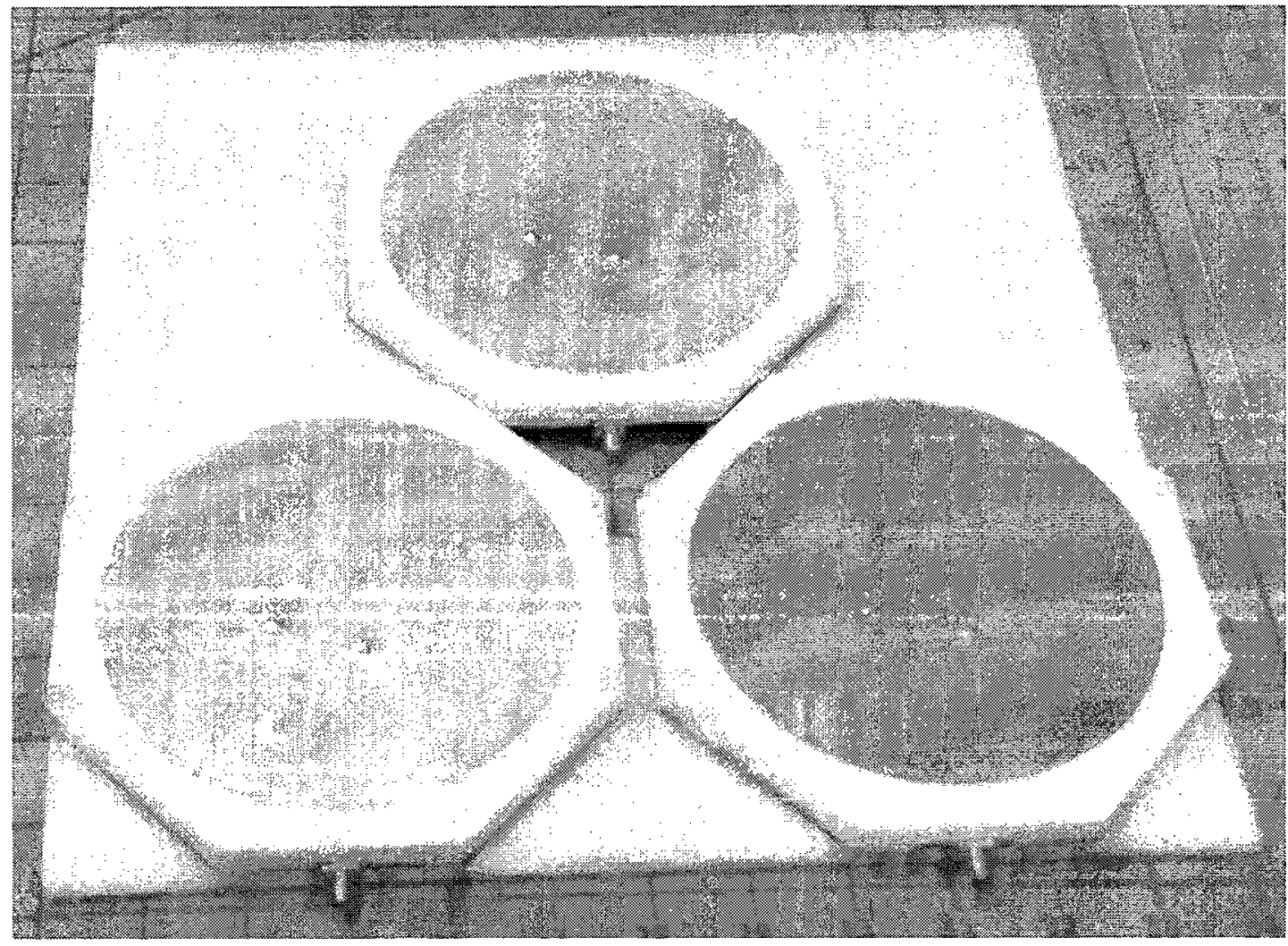

Figure 4.29: Fabricated Patch Antenna Interferometer Array

\subsubsection{Mutual Coupling}

The mutual coupling between the patch antennas was determined by connecting two of the antenna ports to a VNA, while the third antenna was terminated with a $50 \Omega$ load. The mutual coupling (S21) results are shown in Figure 4.30. 




Figure 4.30: Mutual Coupling between two Patch Antennas in the Interferometer Configuration

The mutual coupling between the two elements is better than $-21 \mathrm{~dB}$ over the band of interest. This shows that there is a very low level of coupling between the antennas.

\subsubsection{Patch Antenna Array Phase and Gain versus Azimuth Angle}

The patch antenna array was measured at CRC to test the phase and gain versus azimuth angle.

One patch antenna was rotated about its axis as was done in the previous section. The difference in this test is that there are two other patch antennas in close proximity. The test configuration is depicted in Figure 4.31. 

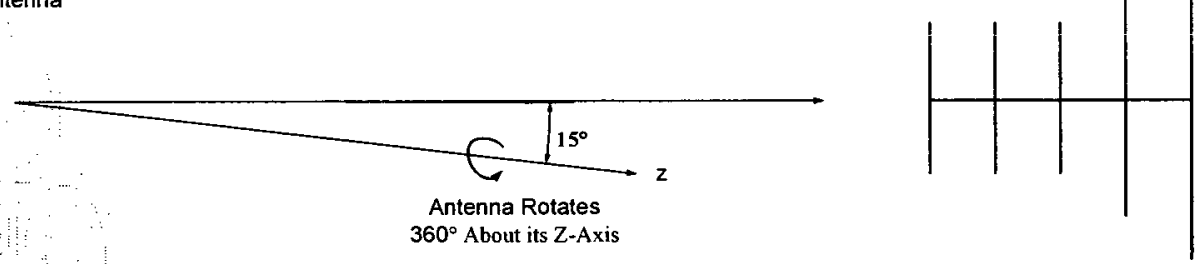

Figure 4.31: Patch Antenna Array versus Azimuth Angle Test Setup at $15^{\circ}$ Elevation Cut

This test was completed to investigate the effect of the other antennas on the phase and gain versus azimuth angle. A comparison of the gain for the single patch and the patch in the interferometer configuration is plotted in Figure 4.32 at a $60^{\circ}$ elevation angle.

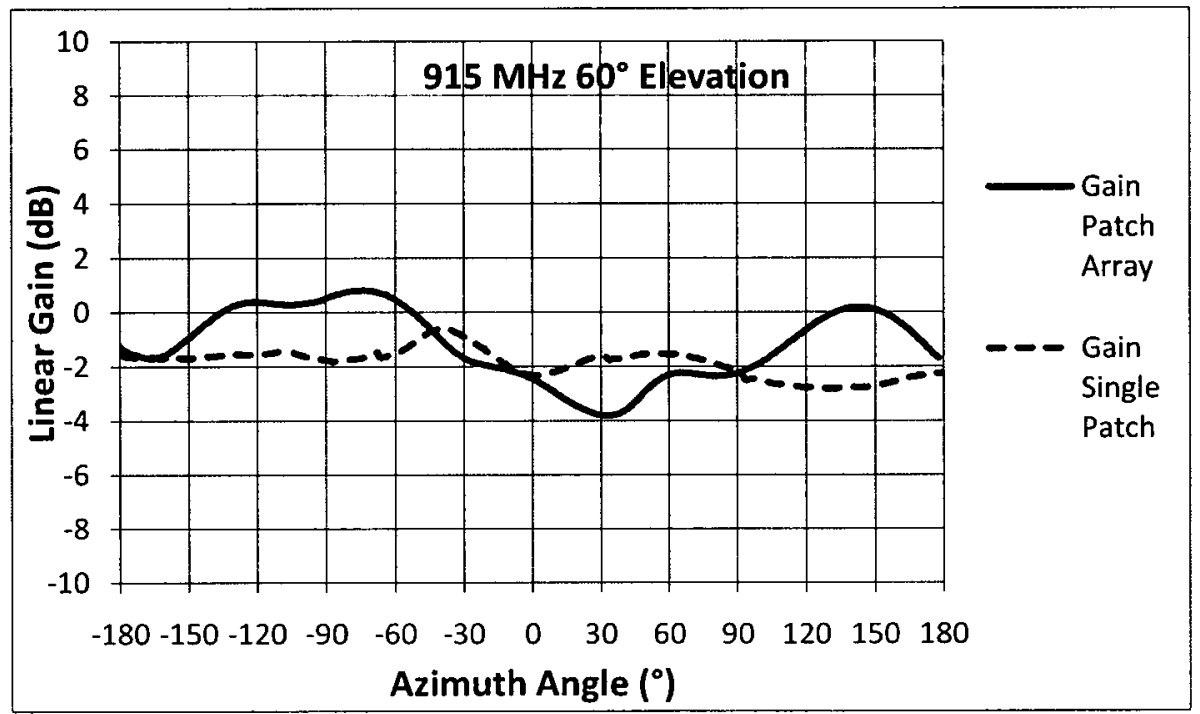

Figure 4.32: Gain Comparison of Single Patch and Patch in an Array Configuration versus Azimuth Angle (915 $\mathrm{MHz}$ at $60^{\circ}$ Elevation Angle)

It is clear that the gain variation has increased due to the presence of the other patch antennas. The standard deviation of the gain is $1.3 \mathrm{~dB}$ compared to $0.3 \mathrm{~dB}$ for the single patch. This is a considerable increase in amplitude variation. 
The phase is also distorted when compared with the measurement results from the single patch.

The phase of the patch versus azimuth angle is plotted in Figure 4.33.

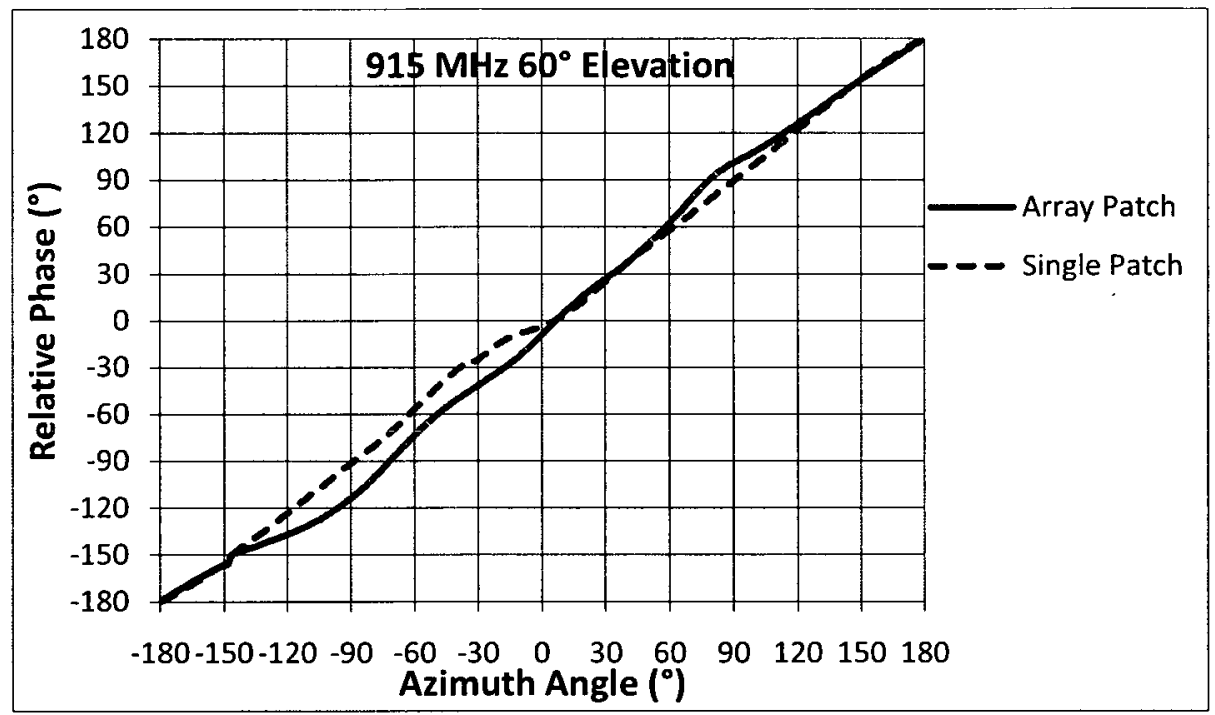

Figure 4.33: Phase Comparison of Single Patch and the Patch in an Array Configuration versus Azimuth Angle (915 MHz at a $60^{\circ}$ Elevation Cut)

The phase of the patch in the array has a maximum deviation of $13.0^{\circ}$ and a standard deviation of $7.2^{\circ}$ at the $60^{\circ}$ elevation cut. This is about double the phase deviation as compared to the single patch. The measurement results of all elevation cuts are summarized in Table 4.3. 
Table 4.3: Patch Array, Gain (Linear) and Phase Deviation versus Azimuth Angle

\begin{tabular}{|l|l|l|l|l|l|l|}
\hline $\begin{array}{l}\text { Elevation } \\
\text { Angle }\end{array}$ & $\begin{array}{l}\text { Average } \\
\text { Gain }(\mathbf{d B})\end{array}$ & $\begin{array}{l}\text { Maximum } \\
\text { Gain }(\mathbf{d B})\end{array}$ & $\begin{array}{l}\text { Minimum } \\
\text { Gain }(\mathbf{d B})\end{array}$ & $\begin{array}{l}\text { Gain } \boldsymbol{\sigma} \\
(\mathbf{d B})\end{array}$ & $\begin{array}{l}\text { Max Phase } \\
\left.\text { Variance( }{ }^{\circ}\right)\end{array}$ & Phase $\sigma\left(^{\circ}\right)$ \\
\hline $0^{\circ}$ & 1.0 & 2.1 & -.1 & 0.9 & 6.0 & 3.8 \\
\hline $15^{\circ}$ & 1.1 & 2.4 & -.2 & 1.3 & 11.3 & 6.7 \\
\hline $30^{\circ}$ & .97 & 2.7 & -1.3 & 2.2 & 13.9 & 9.8 \\
\hline $45^{\circ}$ & .3 & 1.8 & -2.3 & 2.8 & 11.5 & 7.9 \\
\hline $60^{\circ}$ & -1.1 & .8 & -3.8 & 2.3 & 12.9 & 7.2 \\
\hline $75^{\circ}$ & -3.8 & -1.8 & -6.5 & 2.1 & 18.1 & 10.2 \\
\hline $90^{\circ}$ & -7.4 & -3.1 & -19.2 & 3.0 & 25.4 & 17.1 \\
\hline
\end{tabular}

Note that although the patch antennas are flat, they significantly distort the incoming signal.

\subsection{Microstrip Patch Antenna Summary}

The patch antenna has successfully been designed and fabricated. The antenna has the radiation pattern shown in Figure 4.34. 


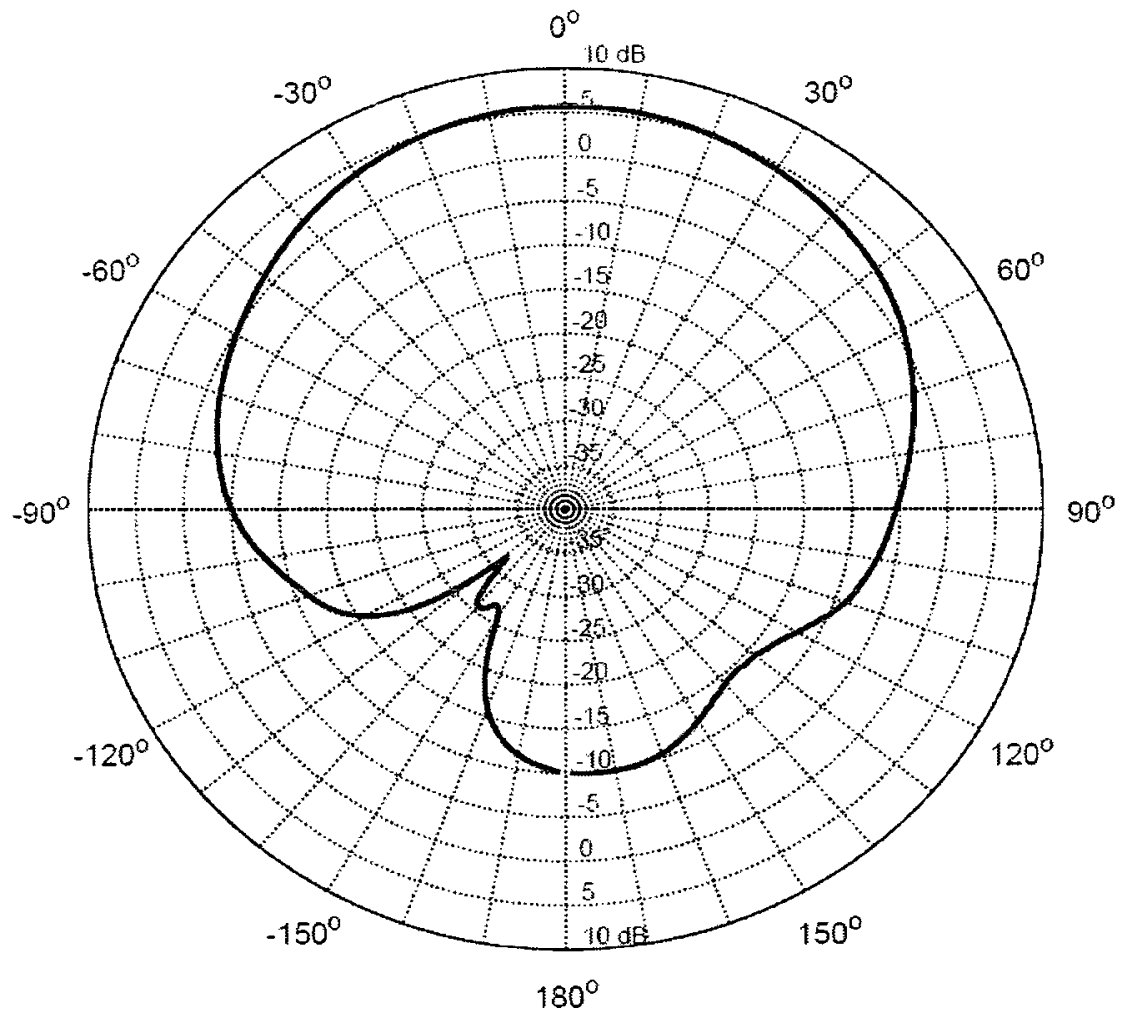

Figure 4.34: UHF RFID Patch Antenna Radiation Pattern (Elevation Cut at 915 MHz)

The patch antenna was successfully designed and performs well in the RFID frequency range with good axial ratio and return loss. The antenna has been size reduced so that it can be configured in an interferometer array with half wavelength spacing. The performance specifications of the patch are described in Table 4.4 . 
Table 4.4: Performance Characteristics of Prototype Patch Antenna

\begin{tabular}{|l||l|}
\hline Frequency of Operation & $902-928 \mathrm{MHz}$ \\
\hline \hline Peak Gain & $6.1 \mathrm{dBic}$ \\
\hline \hline Polarization & RHCP \\
\hline \hline Axial Ratio (Maximum) $\left(0^{\circ}-60^{\circ}\right)$ & $2.2 \mathrm{~dB}$ \\
\hline 3 dB Beamwidth & $44^{\circ}$ \\
\hline Front to Back Ratio & $-15.8 \mathrm{~dB}$ \\
\hline Return Loss Minimum $(50 \Omega$ reference) & $25 \mathrm{~dB}$ \\
\hline Size (Octagon Sides) & $6.12 \mathrm{~cm}$ \\
\hline Thickness & $0.9525 \mathrm{~cm}\left(3 / 8^{\prime \prime}\right)$ \\
\hline
\end{tabular}

The effect of the array configuration on the phase and amplitude deviation of the antenna is of interest. Even though the antenna is flat and the mutual impedance between the elements is better than $20 \mathrm{~dB}$, the phase and amplitude deviation shows a considerable increase. For example, at a $60^{\circ}$ elevation angle the single antenna has a phase versus azimuth angle standard deviation of $3.6^{\circ}$. When the same antenna is placed in the array configuration the standard deviation increases to $7.2^{\circ}$. From the DOA error analysis results in Chapter 3 Table 3.1 the direction of arrival estimation will then have a standard deviation somewhere in the 2 to $3^{\circ}$ range. Calibration of the interferometer array is required to obtain more precise DOA estimation. 


\section{Chapter 5}

\section{Quadrifilar Helix Antenna Array}

The Quadrifilar Helix Antenna (QFHA) is a derivative of the helical antenna and was first developed in the 1960's [15]. The antenna is a popular choice for low earth orbit satellites, because it exhibits excellent circular polarization purity, broad bandwidth, and pattern shaping ability. In a low earth orbit application it is efficient to have maximum gain pointing at the horizon, where the path distance is greatest. The antenna gain can be reduced when the satellite is directly overhead a base station. An example of a satellite QFHA and its radiation pattern is shown in Figure 5.1.
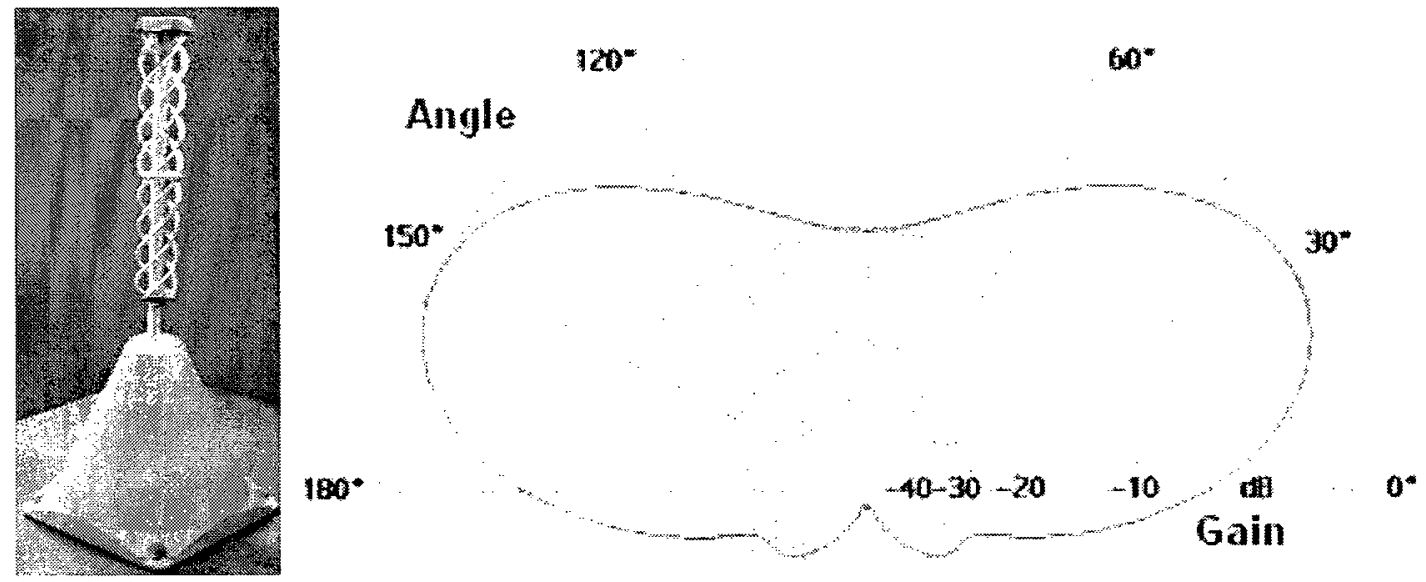

Figure 5.1: Surrey Satellite Technology - S-Band Quadrifilar Helix Antenna (Left) Elevation Pattern (Right) [16]

The Surrey satellite antenna in Figure 5.1 operates in the $2 \mathrm{GHz}$ frequency region. The peak gain of the antenna is $60^{\circ}$ off boresight, and the gain is reduced by $10 \mathrm{~dB}$ at boresight.

This chapter provides details of the QFHA, the benefits of a shaped radiation pattern for an RFID system, and the steps involved in designing the antenna. A prototype of the antenna was built and 
its performance is documented in this chapter. Due to the QFHA's omni-azimuthal radiation pattern it is also proposed that it be used in a direction finding interferometer array. The performance of the QFHA in an interferometer array is interesting, since the 3-D antenna structures in close proximity may cause significant phase and amplitude distortion to each other.

\subsection{QFHA Geometry}

The QFHA consists of a ground plane, four helical elements, and a structure to support the helical elements (typically a rod or tube). There are two types of QFHA which are classified by the feeding arrangement. A Type I QFHA is excited at four ports with equal amplitude and relative phases of $0^{\circ}, 90^{\circ}, 180^{\circ}$ and $270^{\circ}$. A Type II QFHA essentially consists of two orthogonal bifilar helices, and only requires excitation at two ports. A Type I QFHA is used in this work and is depicted in Figure 5.2.

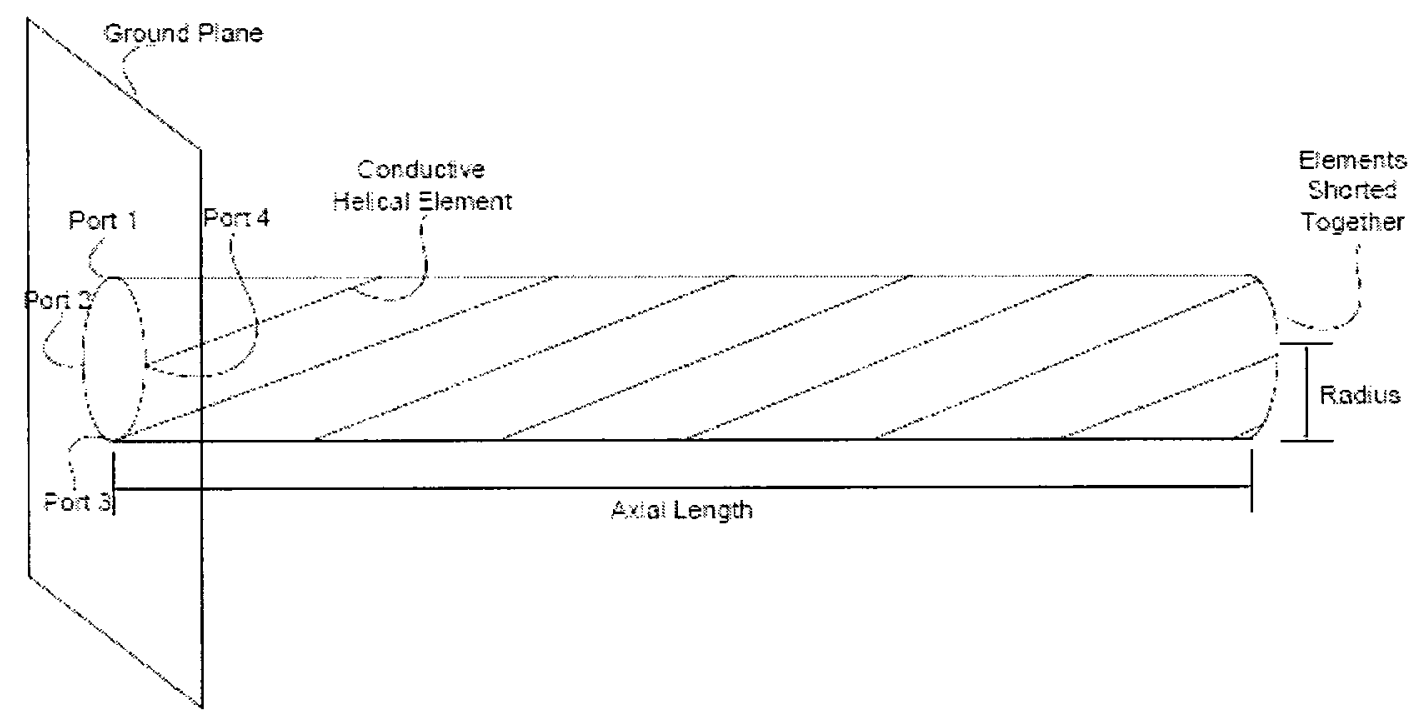

Figure 5.2: Type I QFHA

The elements shown in Figure 5.2 are shorted together at the far right. 
The polarization of the antenna can be either Left Hand Circular Polarization (LHCP) or Right Hand Circular Polarization (RHCP) depending on the direction the helices are wound and phased at each port.



a)

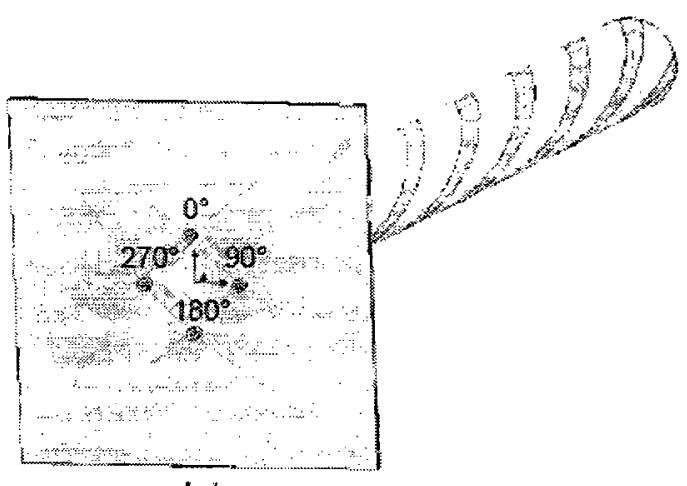

b)

Figure 5.3: QFHA, RHCP Clockwise Windings (a) LHCP Counter-Clockwise Windings (b)

Figure 5.3 depicts the correct helical winding and phasing at the ports for LHCP and RHCP. The geometrical design parameters of the QFHA are very similar to the helical antenna and include diameter $\mathrm{D}$, pitch distance $\mathrm{S}$, axial length $\mathrm{L}$, conductor diameter $2 \mathrm{a}$, and the number of turns $\mathrm{N}$ as shown in Figure 5.4. 




Figure 5.4: Helical Antenna Parameters [11]

The geometrical properties of the helix then form the following relations:

$$
\begin{gathered}
C=\pi D \\
\alpha=\tan ^{-1}\left(\frac{S}{C}\right) \\
L_{0}=\sqrt{S^{2}+C^{2}} \\
L_{n}=N L_{0}
\end{gathered}
$$

Where $\mathrm{C}$ is the circumference of the helix, $\alpha$ is the pitch angle, $L_{0}$ is the length of wire between turns, and $L_{n}$ is the total wire length. By correctly choosing the geometrical parameters, an antenna with the appropriate operating frequency, bandwidth, and radiation pattern can be designed. 


\subsection{Understanding the Ideal Radiation Pattern Shape}

In a passive UHF RFID system, the gain of the antenna is essential for reaching the maximum read distance. An antenna pattern that has a peak gain pointing towards areas with the most pathloss is always ideal. While the traditional RFID patch antenna delivers peak gain at boresight there are many applications that require an antenna with a different pattern shape. Consider the situation where an RFID antenna is centered above the desired coverage area as shown in Figure 5.5 .

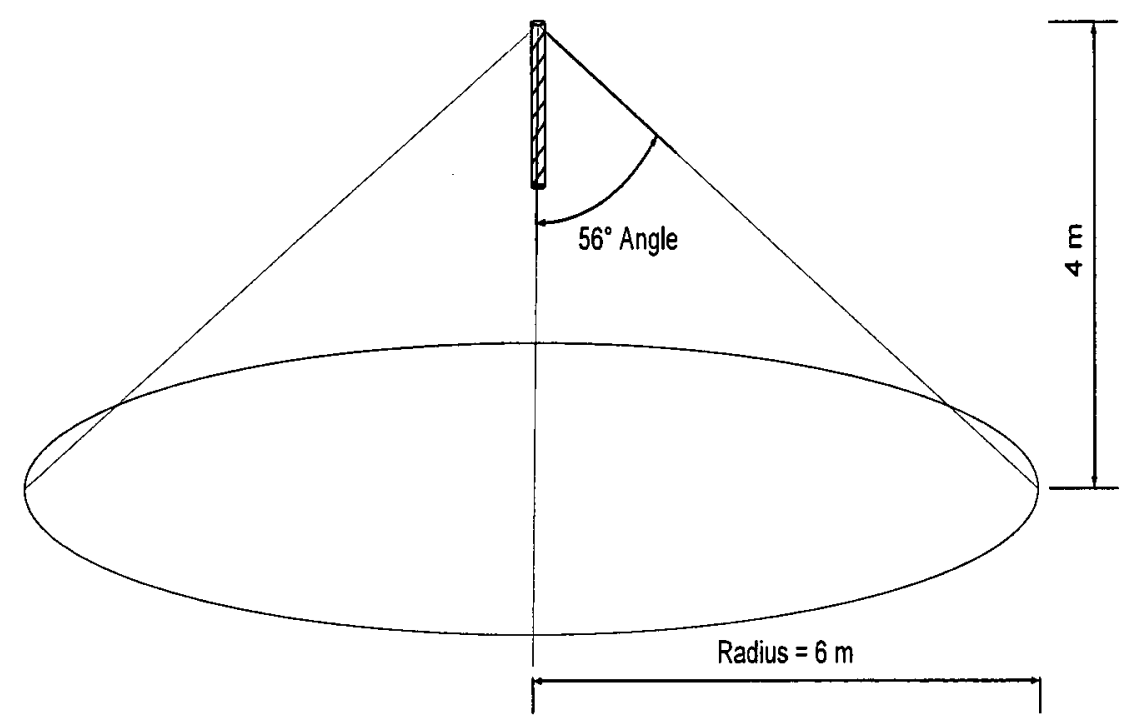

Figure 5.5: RFID Application where the Reader Antenna is Centered Above the Coverage Area

The antenna is placed at a height of 4 meters and the desired coverage area below is a circle with a 6 meter radius. The corresponding elevation angle with maximum path loss is then $56^{\circ}$. The most efficient antenna pattern would be omni-azimuthal and have an elevation pattern with peak gain at $56^{\circ}$. 
A link budget analysis was completed to obtain the minimum gain requirements at all elevation angles. Passive RFID tags are limited by the forward link budget, which means that the amount of energy available to the tag is the limiting factor. Assuming that a passive UHF RFID tag has a threshold power $\left(\mathrm{Tag}_{\text {threshold }}\right)$ of $30 \mathrm{uW}(-15 \mathrm{dBm})$ [2], then the required antenna gain for a particular distance can be found. The following equations were used in the link budget calculation to create Figure 5.6.

$$
\begin{gathered}
G_{\text {Required }}=T a g_{\text {threshold }}-G_{\text {tag }}-T x_{\text {power }}+P_{\text {loss }}(d B) \\
P_{\text {loss }}=20 * \log \left(\frac{\lambda}{4 * \pi * D}\right) \\
\text { Tag threshold } \\
=10 * \log (30 u W)(d B)
\end{gathered}
$$

The $T x_{\text {power }}$ is limited by the FCC as $30 \mathrm{dBm}, G_{\text {tag }}$ is assumed to be $0 \mathrm{~dB}, D$ is the path distance from the antenna to the tag, $G_{\text {Required }}$ is the required gain of the antenna for communication with the RFID tag, $P_{\text {loss }}$ is the path loss of the signal. The minimum required gain has been plotted against elevation angle in Figure 5.6. 


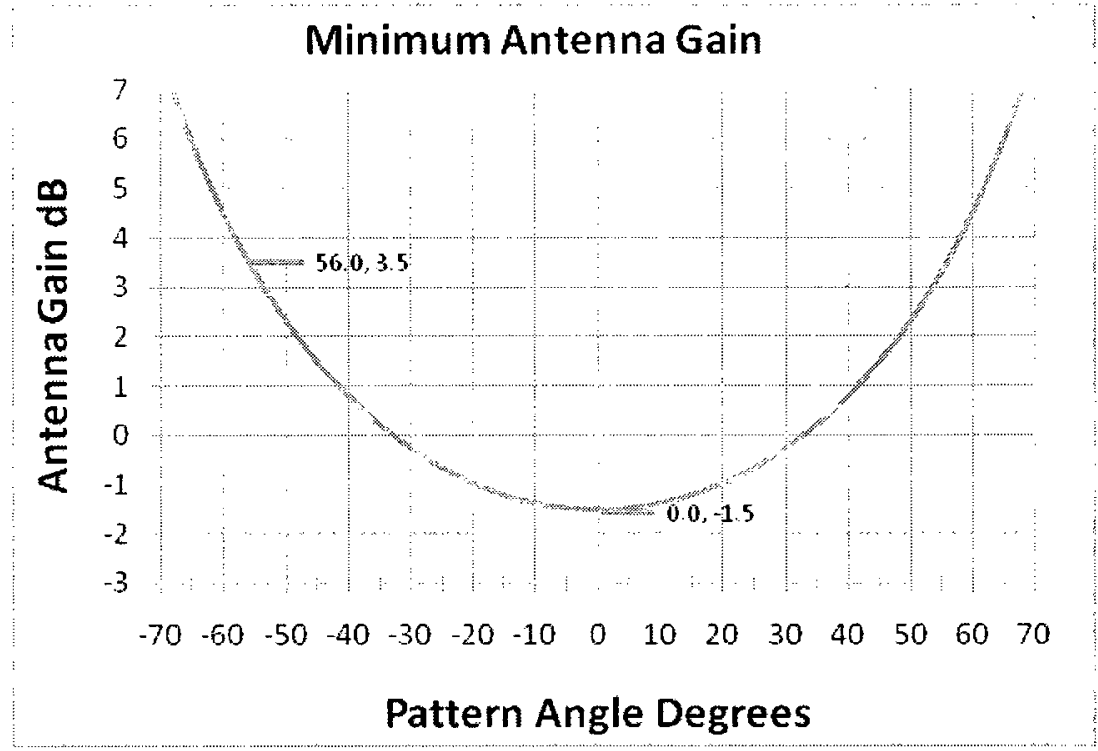

Figure 5.6: Required Gain versus Elevation Angles for the Situation Depicted in Figure 5.5 At boresight $\left(0^{\circ}\right)$ the required gain is at least $-1.5 \mathrm{~dB}$, while at $56^{\circ}$ the minimum required gain is 3.5 dB. Literature [17],[18] and computer simulations lead to the conclusion that a QFHA would be capable of producing this desired radiation pattern.

\subsection{The QFHA Radiation Pattern Theory}

The helix antenna can operate in a number of different modes; most commonly the helix antenna is designed to operate in either normal or end-fire mode which is depicted in Figure 5.7. 


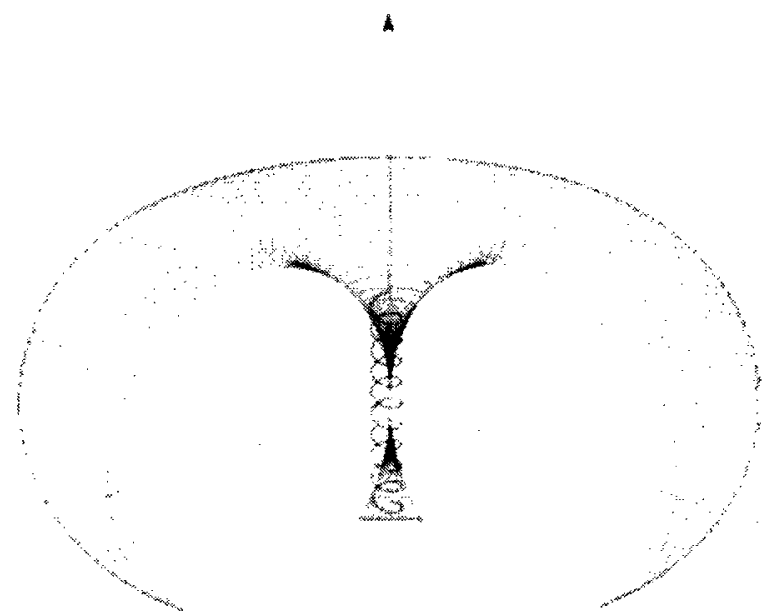

a)

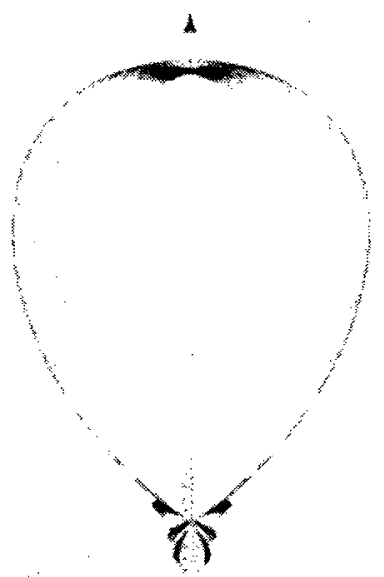

b)

Figure 5.7: Monofilar Helix, Normal Mode (a) Endfire (Axial) Mode (b) [11]

Significant experimental data has been created for the helical antenna, and it has been found that the normal mode helix is achieved when both the length and circumference of the antenna is small compared to a wavelength $\left(N L_{0} \ll \lambda_{0} \gg C\right)$. The axial mode is excited when the geometry is large fractions of a wavelength. The circumference of the axial mode helix is achieved when $C / \lambda_{0}$ is around unity and the pitch distance $\mathrm{S}$ is about $\lambda_{0} / 4$.

The helical chart by Kraus[13] Figure 5.8, shows the different modes of operation for the helical antenna as a function of pitch spacing and circumference. 


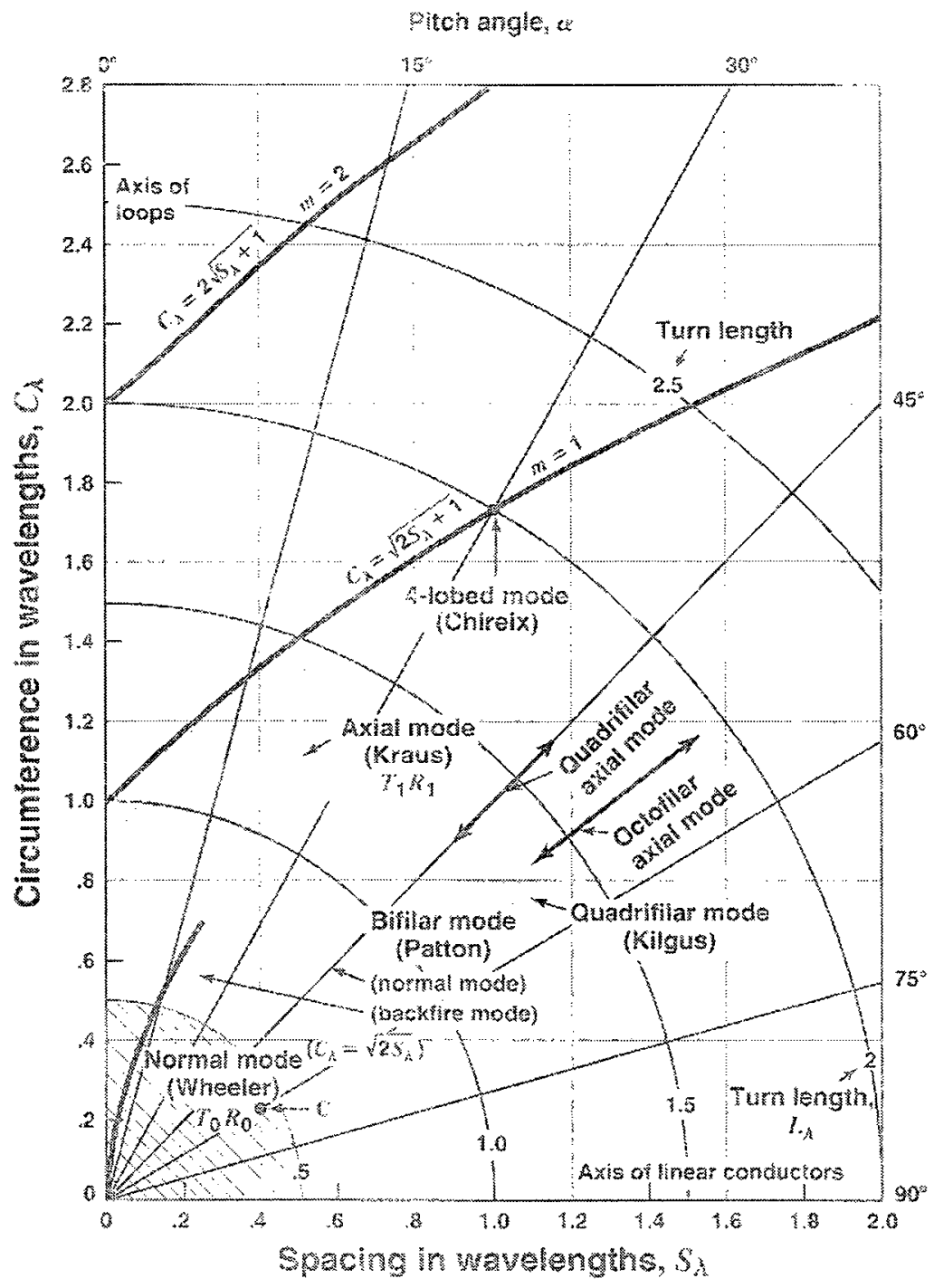

Figure 5.8: Helix Chart Showing the Location of Different Modes of Operation as a

\section{Function of the Helix Dimensions (Diameter, Spacing, and Pitch Angle). [13]}

The normal mode of operation can be seen in the bottom left hand corner where the dimensions of the helix are small. The axial mode (Kraus) is shown in the grey area where the circumference in wavelengths is unity and the spacing is around $\lambda_{0} / 4$. In the early 1970 's, Kilgus did extensive experimentation on the QFHA and found a region that produces a shaped radiation pattern. This 
area is also depicted in Figure 5.8 as the grey region labeled "Quadrifilar mode (Kilgus)". Kilgus presents various curves and experimental shaped beam pafterns in [17]. In his experimental data the term $\mathrm{k}$ is used, which is the ratio of the radius in wavelengths $\left(r_{0}\right)$ and the pitch distance in wavelengths $(p)$.

$$
k=\frac{r_{0}}{p}
$$

The region of shaped beam operation is shown in Figure 5.9.

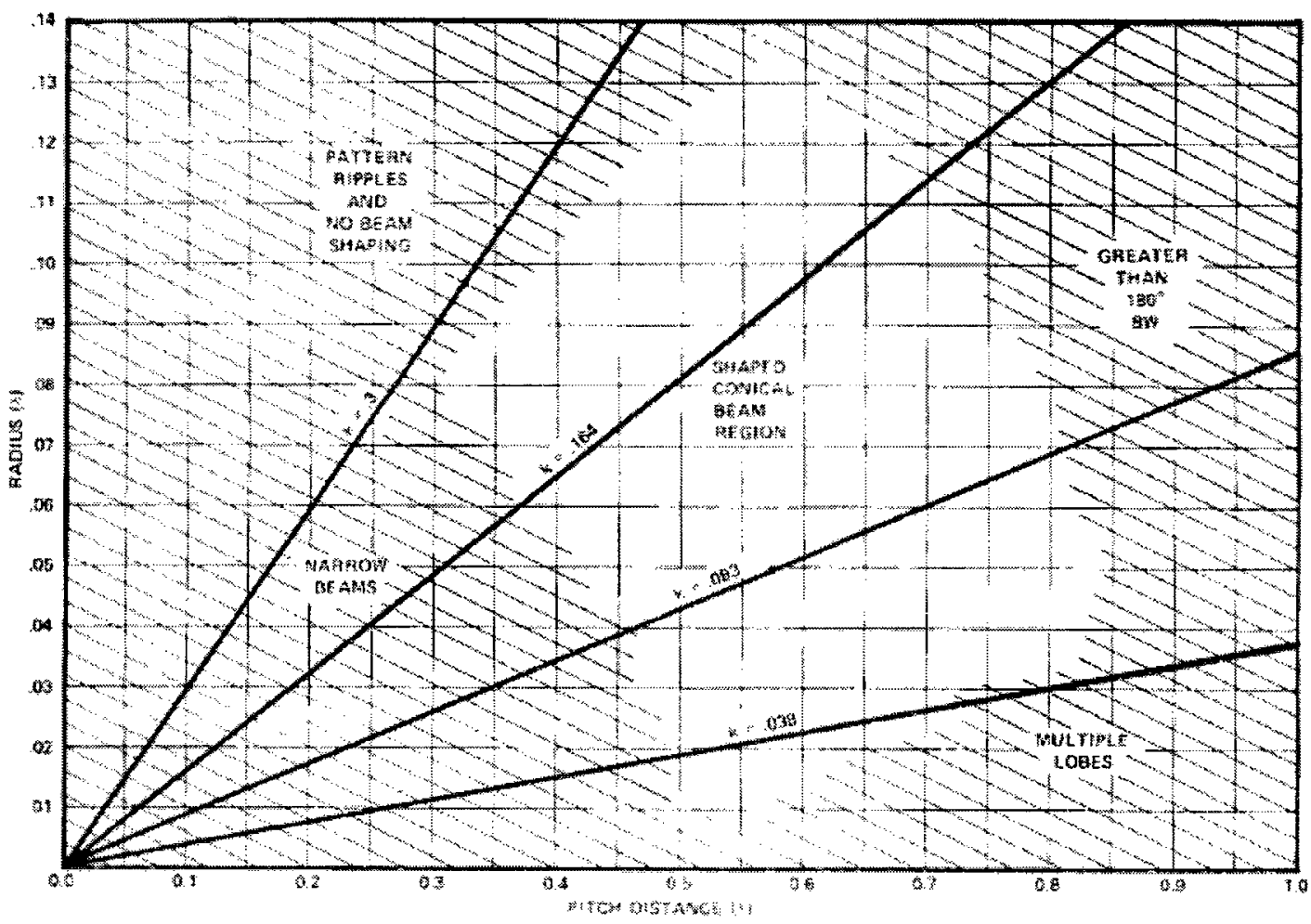

Figure 5.9: Region of Shaped Conical Beam Performance [17]

Several experimental charts and tables in [17] and [18] describe the shaped patterns that were achieved for different pitch distance, radius, and number of turns. Kilgus defines the shaped beam of the antenna with reference to Figure 5.10. 


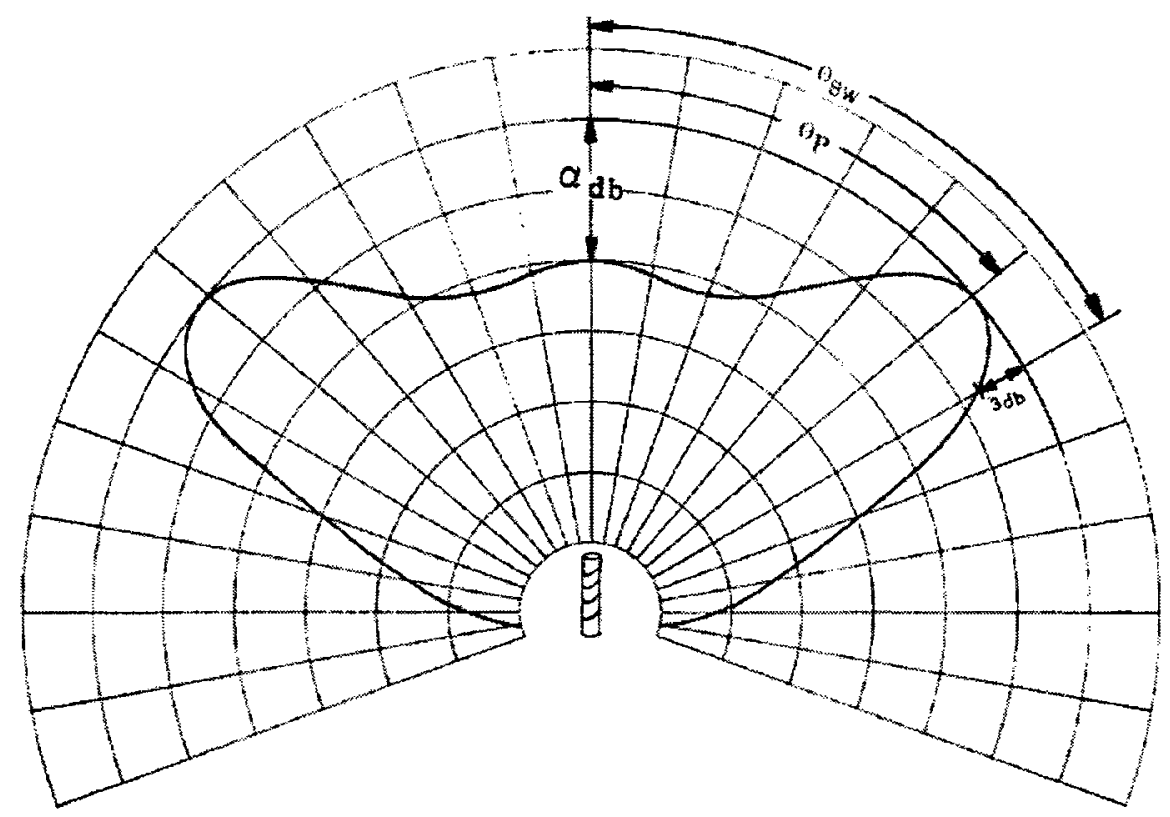

Figure 5.10: Elevation Cut of a Shaped Radiation Pattern [17]

In Figure $5.10, \theta_{B W}$ refers to the $3 \mathrm{~dB}$ point outside the pattern maximum, $\theta_{p}$ is the angle between the $\mathrm{z}$ axis and the peak gain, and $\alpha_{d B}$ is the depth of the pattern minimum $\left(\theta=0^{\circ}\right)$ relative to the pattern maximum at $\theta_{p}$. Several charts have been created describing the shaped beam pattern as a function of geometry with the parameters $\theta_{B W}, \alpha_{d B}$, and $\theta_{p}$. Figure 5.11 is a chart that is relevant to the work in this thesis, and describes the radiation pattern of a two turn QFHA. 


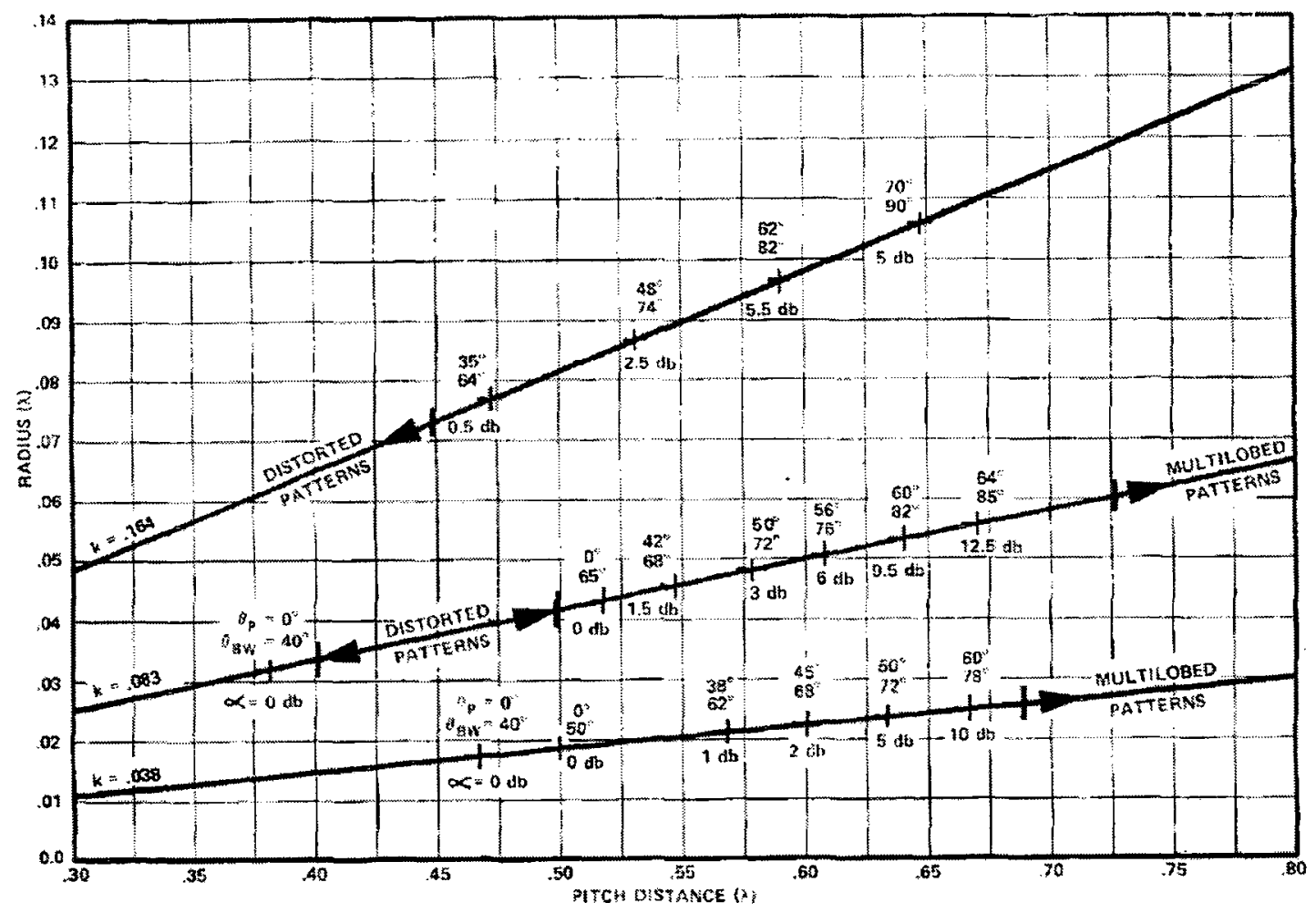

Figure 5.11: Radiation Pattern Characteristics for Quadrifilar with $N=2$ [17]

In this chart there is a region around radius $.05 \lambda_{0}$ and pitch distance $.6 \lambda_{0}$ where the peak gain is about $56^{\circ}$ and the gain is reduced by $5 \mathrm{~dB}$ at boresight. This is the radiation pattern that is desirable.

\subsection{Quadrifilar Helix Design}

Availability and cost of materials dictated many choices in the QFHA design. The major design choices related to material, sizing, and feeding technique are described below:

1. Material Selection Rod/Tube: The QFHA was designed on a Teflon rod because it is low cost and has excellent electrical properties. Teflon has a loss factor of 0.001 and a dielectric constant of 2.1. A Teflon rod with a $2.54 \mathrm{~cm}$ (1") diameter has been chosen 
since it is a standard size rod that is readily available. Computer simulations prove that this diameter can provide the desired radiation pattern.

2. Feeding Technique: A QFHA can be fed at the top or bottom. It was decided that the QFHA would be fed at the bottom of the antenna where the microstrip feed network Printed Circuit Board (PCB) could also be used as a ground plane for the antenna.

3. Ground Plane and Feed network: A desirable characteristic of the QFHA is that a large ground plane is not necessary [17]. A standard .062" FR4 substrate has been chosen for the PCB feed network/ground plane because it is inexpensive. The size of the ground plane was determined to be $10 \mathrm{~cm}^{2}$ in order to accommodate the feed network

4. Helical Conductor Width: For the prototype antenna, the helical conductor, was made with copper tape. Copper tape was chosen for ease and flexibility in construction and a Standard $0.635 \mathrm{~cm}(1 / 4)$ width copper tape was used for this antenna.

\subsubsection{Shaping the Radiation Pattern}

Correctly shaping the radiation pattern is the most difficult task in the design of the QFHA. In the past this was a serious undertaking which would require significant experimentation. However, with the advent of EM computer simulation tools and optimization, the design process has become much easier. The experimental curves in [17], and [18] offer insight into the behavior of the QFHA, but only provide accurate design data for the specific documented case. Simulations were completed in HFSS to create a similar chart to Figure 5.11 using only a Teflon rod, a $10 \mathrm{~cm}^{2}$ ground plane and $0.635 \mathrm{~cm}$ wide helical arms. 


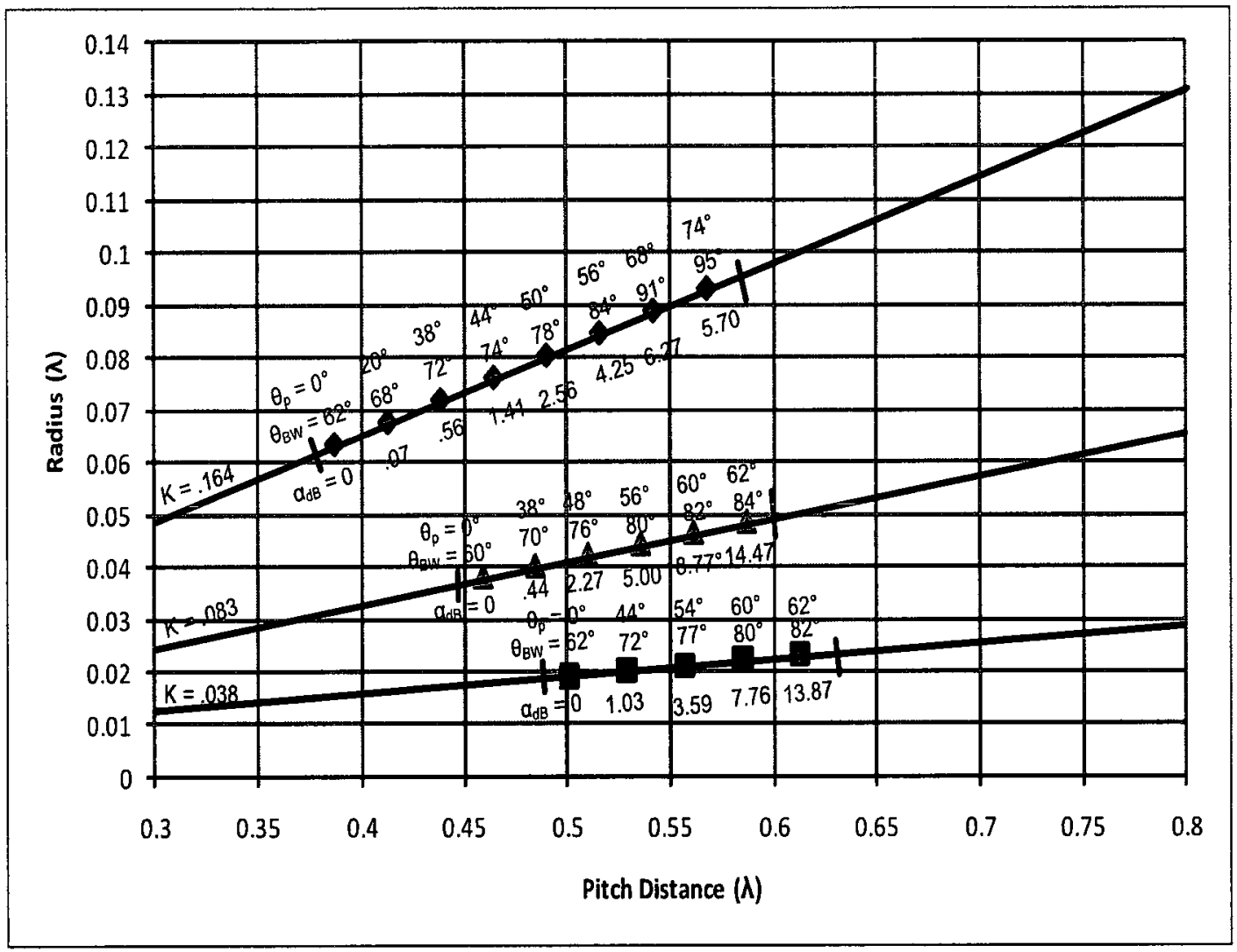

Figure 5.12: Radiation Pattern Characteristics with a Teflon $\operatorname{Rod}$ for $N=2$

From Figure 5.12, an area where the radiation pattern corresponds well to the ideal pattern is at a radius of $.045 \lambda_{0}$ and pitch distance of $.536 \lambda_{0}$. This point was chosen for the initial antenna design and corresponds to a $915 \mathrm{MHz}$ antenna with a total axial length and radius of $35.1 \mathrm{~cm}$ and $1.46 \mathrm{~cm}$ respectively. To accommodate a $1.27 \mathrm{~cm}$ radius Teflon rod, the antenna design was slightly modified to a length of $36 \mathrm{~cm}$ and radius of $1.27 \mathrm{~cm}$. 


\subsection{Verification of the HFSS Model}

Simulating the antenna structure is much faster and less costly than creating expcrimcntal prototypes and testing. To verify the HFSS model an antenna was fabricated and tested. The HFSS model and fabricated antenna can be seen in Figure 5.13.

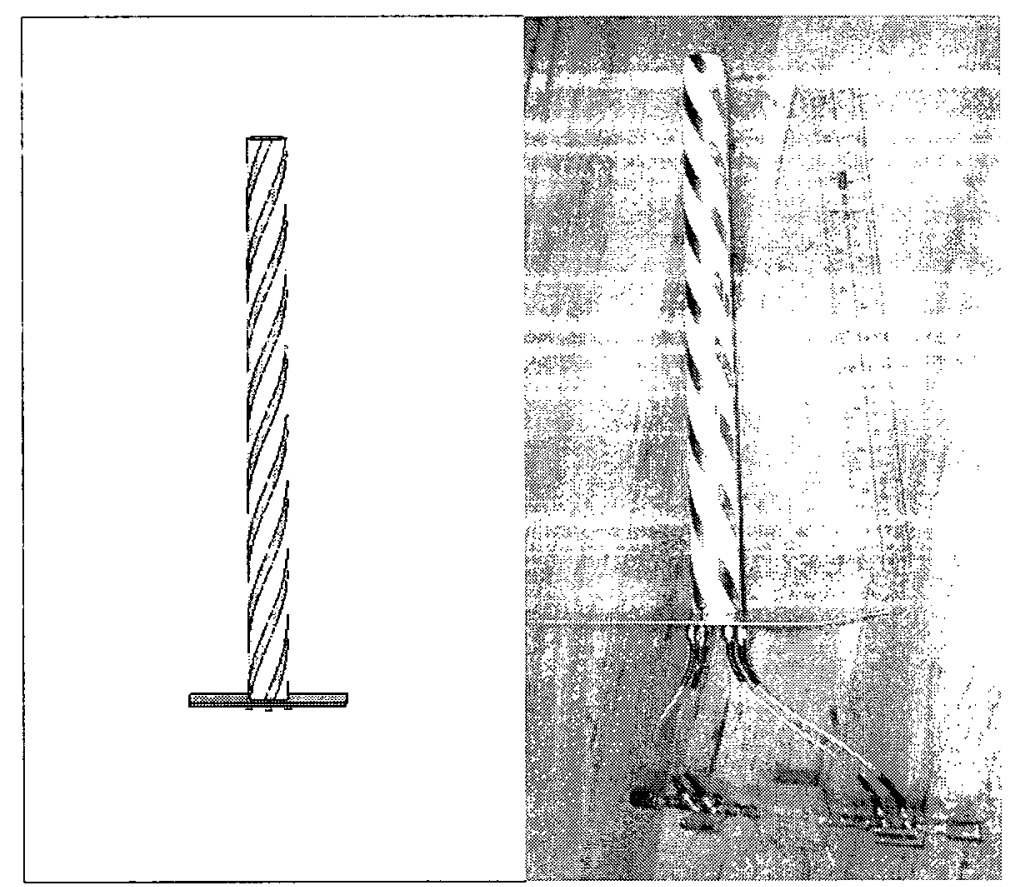

Figure 5.13: QFHA, HFSS Model (Left) Fabricated Antenna (Right)

To construct the QFHA, a thin plastic sheet was used which makes it easier to wind the copper tape onto the Teflon rod. Copper tape was first placed on the plastic sheet as shown in Figure 5.14, and then the entire sheet was then rolled onto the Teflon rod. 


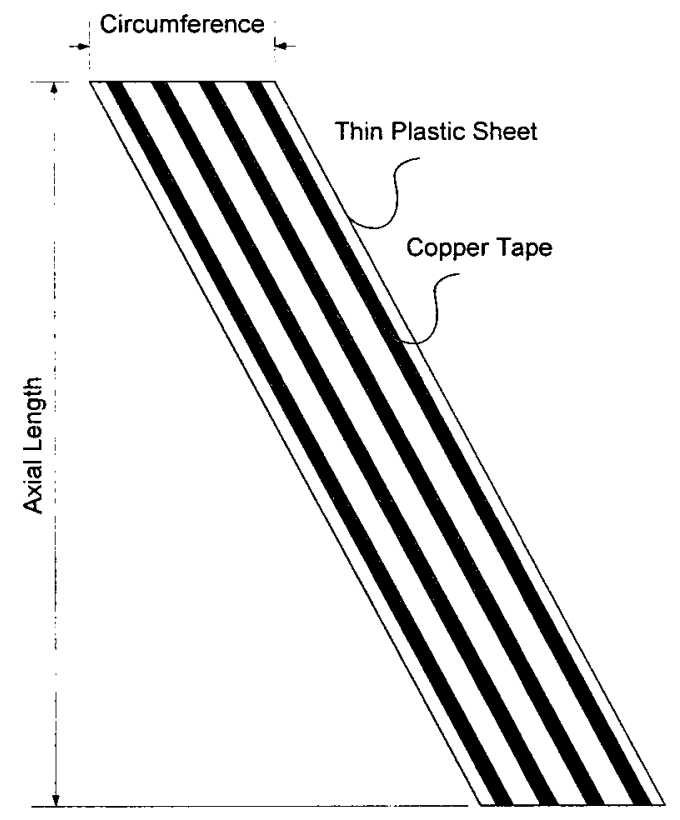

Figure 5.14: Plastic Sheet with Copper Tape

The Teflon rod was fastened to the ground plane $\left(10 \mathrm{~cm}^{2}\right.$ piece of $62 \mathrm{mil} \mathrm{FR4),} \mathrm{and} \mathrm{four} \mathrm{holes}$ were drilled in the ground plane so that four SMA connectors could be soldered on. Next the probes of the SMA connectors were soldered to the helical elements. The feed network is composed of commercial components from Mini-Circuits. A $180^{\circ}$ hybrid (ZFSCJ-2-4) and two $90^{\circ}$ hybrids (ZX10Q-2-12) create the correct phasing and amplitude at the four ports. The HFSS model and the fabricated antenna have the parameters given in Table 5.1. 
Table 5.1: Parameters of the QFHA Antenna Fabricated and HFSS Model

\begin{tabular}{|l|l|}
\hline QFīA Design Parameter & Vaiue \\
\hline \hline Turns & 2 \\
\hline Radius & $1.27 \mathrm{~cm}\left(.5^{\prime \prime}\right)$ \\
\hline Axial Length & $36 \mathrm{~cm}$ \\
\hline Rod Material & Teflon $(\mathrm{Dk}=2.1, \mathrm{Df}=.001)$ \\
\hline Ground Plane & $10 \mathrm{~cm}^{2}$ \\
\hline Helical Element Strip Width & $0.635{\mathrm{~cm}\left(0.25^{\prime \prime}\right)}$ \\
\hline
\end{tabular}

\subsubsection{Radiation Pattern}

The radiation pattern of the fabricated antenna was measured in the anechoic chamber at Carleton

University, and has been compared against the simulated pattern of an ideal lossless HFSS model.

The comparison can be seen in Figure 5.15. 


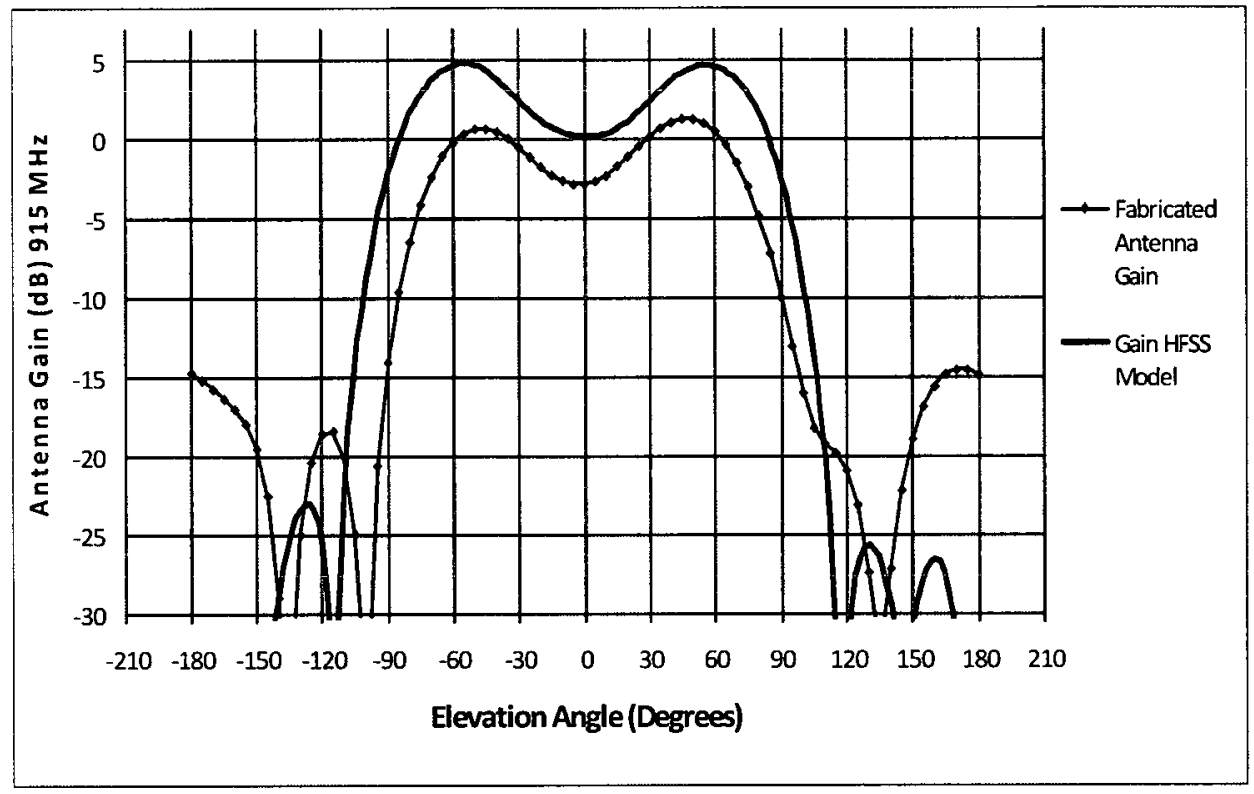

Figure 5.15: Antenna Elevation Pattern at $915 \mathrm{MHz}$, HFSS Ideal Simulation and Measurement

The simulated radiation pattern easily meets the gain requirements mentioned earlier with peak gain of $4.74 \mathrm{dBic}$ occurring at $56^{\circ}$. Unfortunately, the fabricated antennas gain is reduced by as much as $5 \mathrm{~dB}$. Also, the pattern shape of the fabricated antenna is slightly different with peak gain occurring at $45^{\circ}$. This can be attributed to the fact that the antenna is homemade, the computer model has not considered all material loss, and the feed network has losses. It is evident that there is a frequency shift between the simulated and measured results, which accounts for the slightly different pattern shape. Despite all this, it is clear that the radiation patterns are similar in shape, which proves that the model can predict the behavior of the real world antenna. 


\subsubsection{Input Impedance}

The HFSS model and the fabricated antenna depicted in Figure 5.13 were used to verify the input impedance of the antenna. The S-parameters of the QFHA were measured with an HP 8720ES network analyzer. The ports were arbitrarily numbered 1 through 4 as can be seen in Figure 5.2. Two ports were measured while the other two ports were terminated with $50 \Omega$ loads as depicted in Figure 5.i6.

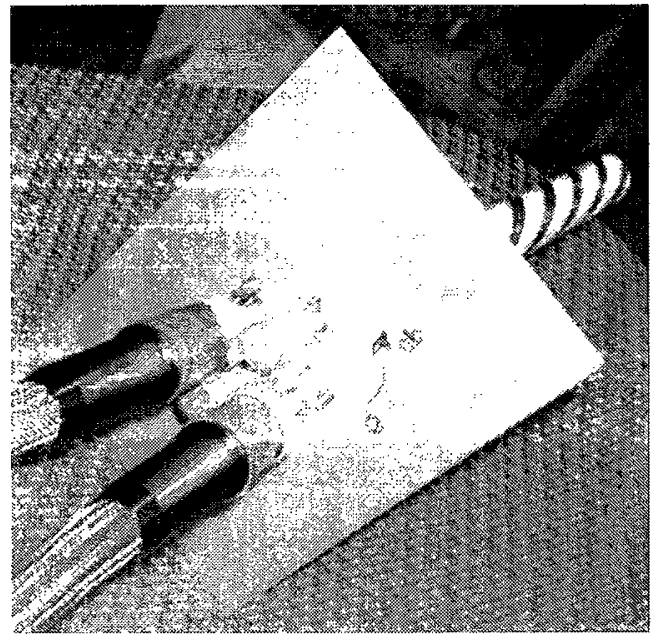

Figure 5.16: S-parameter measurement using a VNA to find $S_{11}$ and $S_{13}$

The results from the S-parameter measurements match very well with simulation results as seen from the plots Figure 5.17, Figure 5.18, and Figure 5.19. 


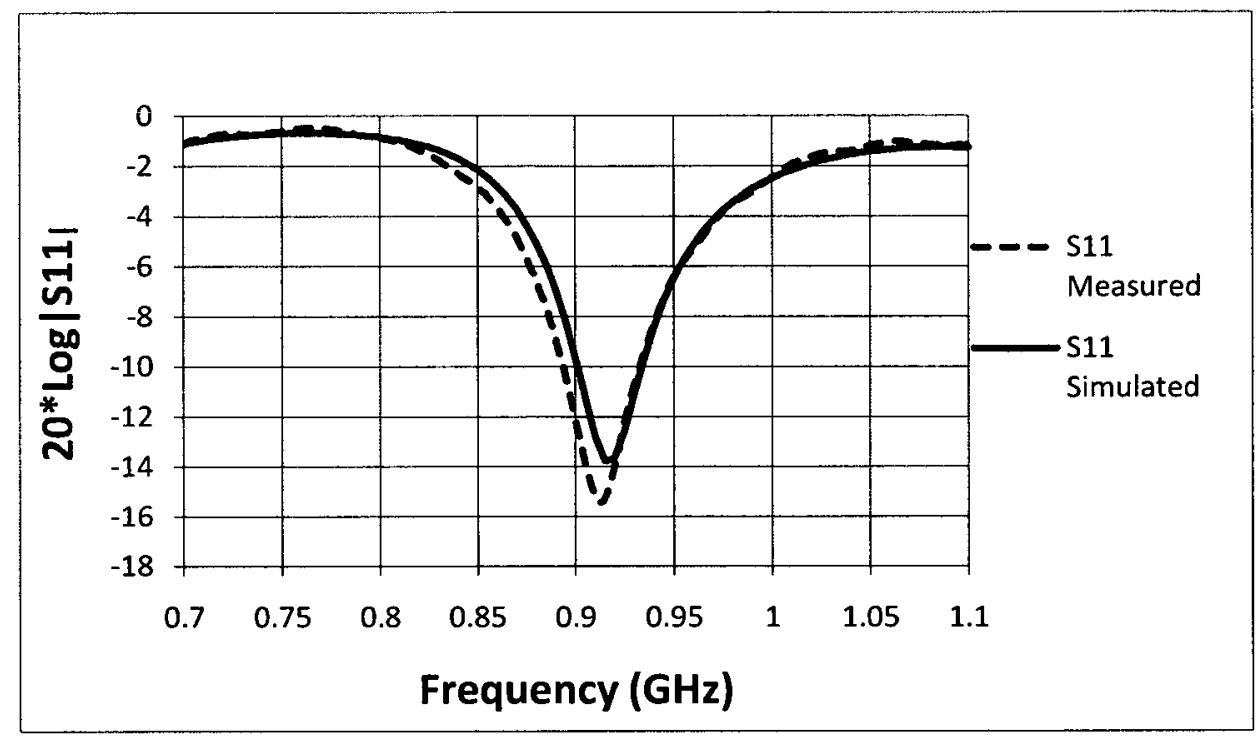

Figure 5.17: Comparison of S11 Simulation (Solid) Measured (Dashed)

Note: $S 11, S 22, S 33, S 44$ results are similar

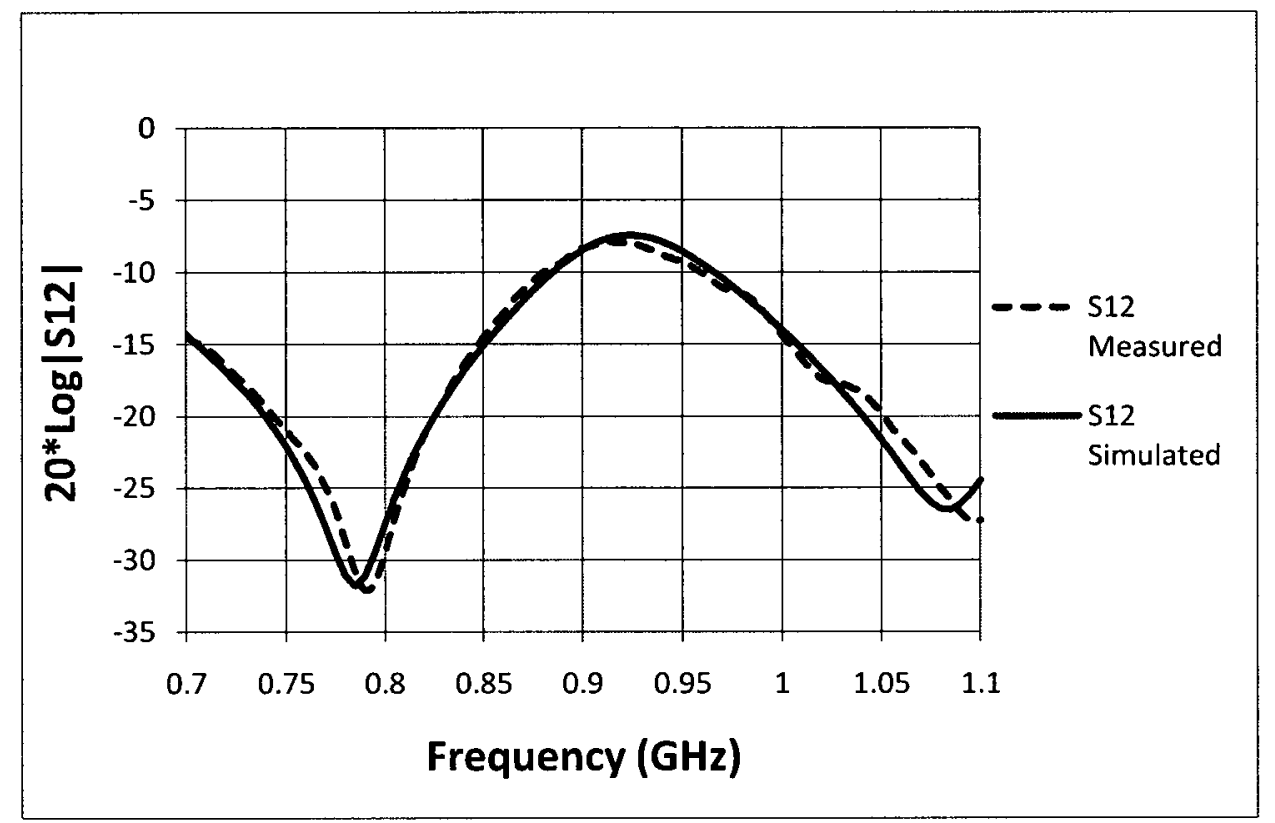

Figure 5.18: Comparison of S12 Simulation (Solid) Measured (Dashed)

Note: $S 12, S 43, S 32$, and $S 41$ results are similar 


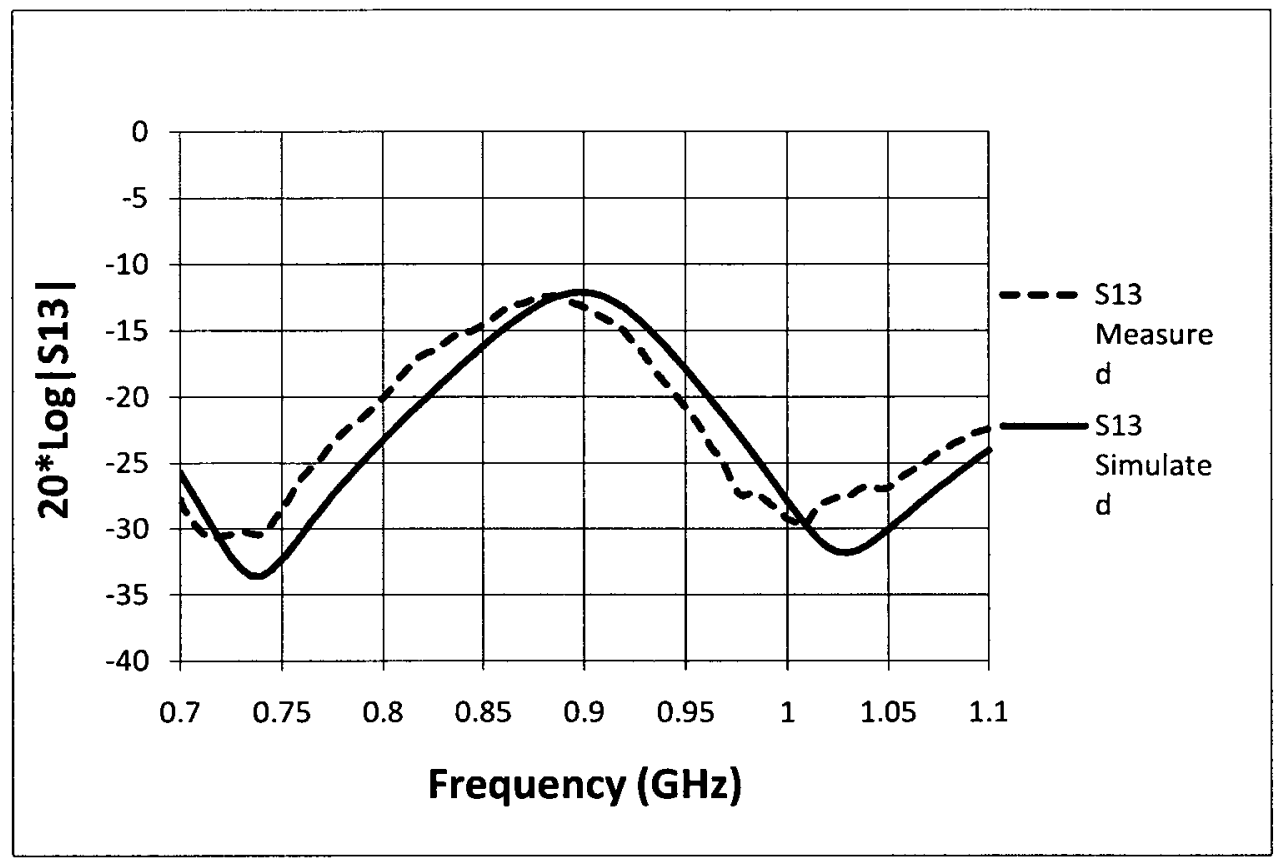

Figure 5.19: Comparison of S13 Simulation (Solid) Measured (Dashed)

Note: $S 13$ and $S 24$ results are similar

By comparing the simulated and measured S-parameters the results are nearly identical, however there is a frequency shift of $10-15 \mathrm{MHz}$. This is only a $2 \%$ shift and within the tolerances of making the antenna by hand.

From the S-parameter measurements it is possible to find the impedance of the antenna. One may believe that the input impedance of the QFHA may be found by measuring the reflection coefficient from one of the antenna ports and using the single port relation of Equation 5.9.

$$
Z_{\text {in }}=\frac{1+S_{11}}{1-S_{11}} Z_{0}
$$

Equation 5.9 cannot be used because the impedance of the antenna is affected by the other port excitations. The impedance of each arm of the QFHA can be found with the following modified version of Equation 5.9 derived in [19]: 


$$
Z_{\text {in }}=\frac{1+S_{11}-S_{13}}{1-S_{11}+S_{13}} Z_{0}
$$

Equation 5.10 has been used to find the impedance of the helical arms of the QFHA and the results are plotted in Figure 5.20 below.

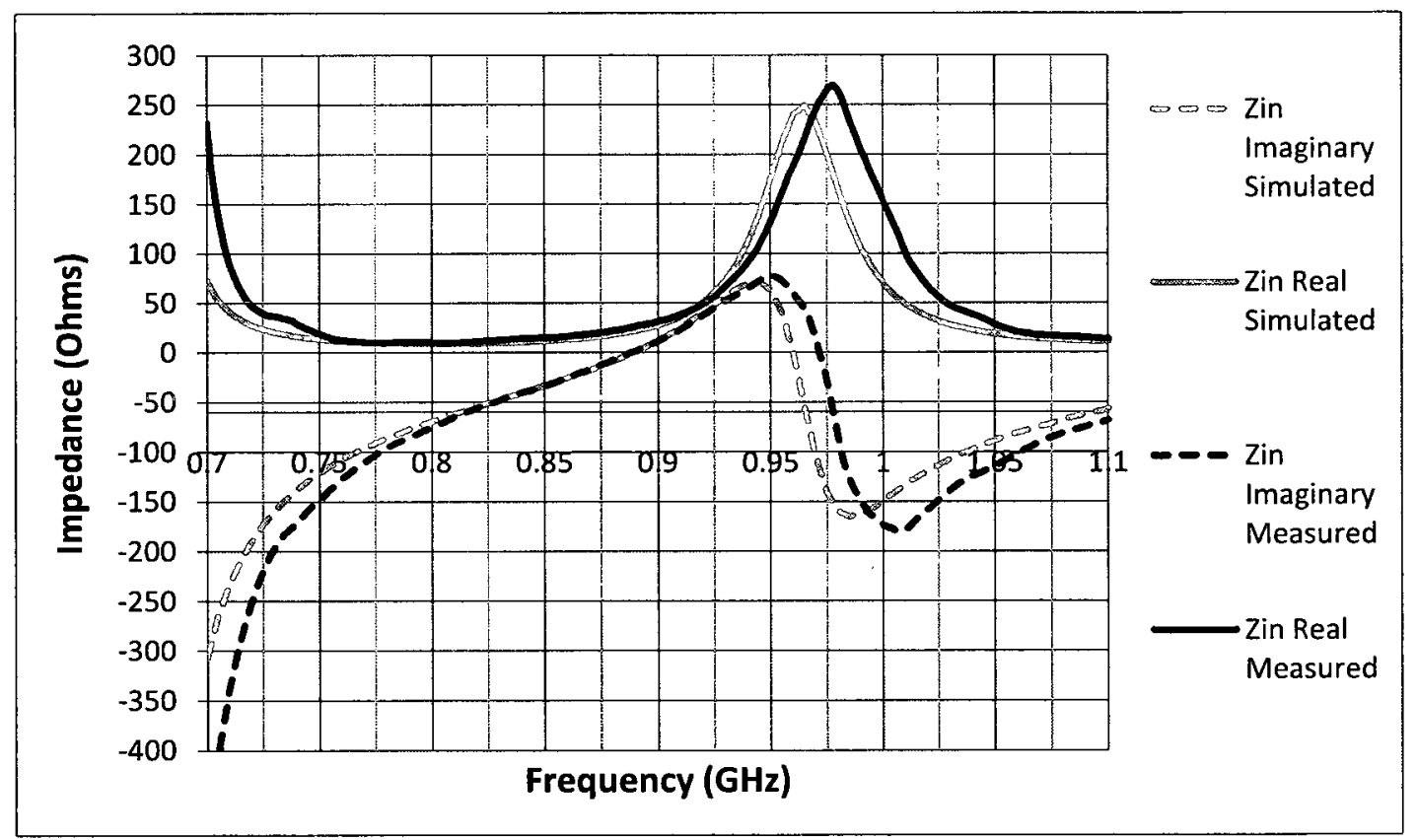

Figure 5.20: Input Impedance of the HFSS Model Compared with the Fabricated QFHA

The impedance of one of the helical arms can be seen in Figure 5.20. The response of the measured antenna is shifted in frequency by approximately $13 \mathrm{MHz}$ from the simulated results. At the center frequency $915 \mathrm{MHz}$ the measured impedance of the fabricated antenna is $42.8+30.1 \mathrm{j}$. This corresponds to a return loss of $10.0 \mathrm{~dB}$ when used with a $50 \Omega$ transmission line, which has been considered acceptable for this prototype antenna.

\subsection{Feed network Design}

The design of the feed network was based on [20], where one $180^{\circ}$ hybrid and two Wilkinson splitters were used. The feed network has been designed using Ansoft HFSS and Agilent ADS, 
and the feed network splits the input signal into four separate ports. Each of the four output ports are 90 degrees out of phase with the adjacent ports, creating excitations at $0^{\circ}, 90^{\circ}, 180^{\circ}$, and $270^{\circ}$. The feed network was manufactured on FR4 substrate and is shown in Figure 5.21.
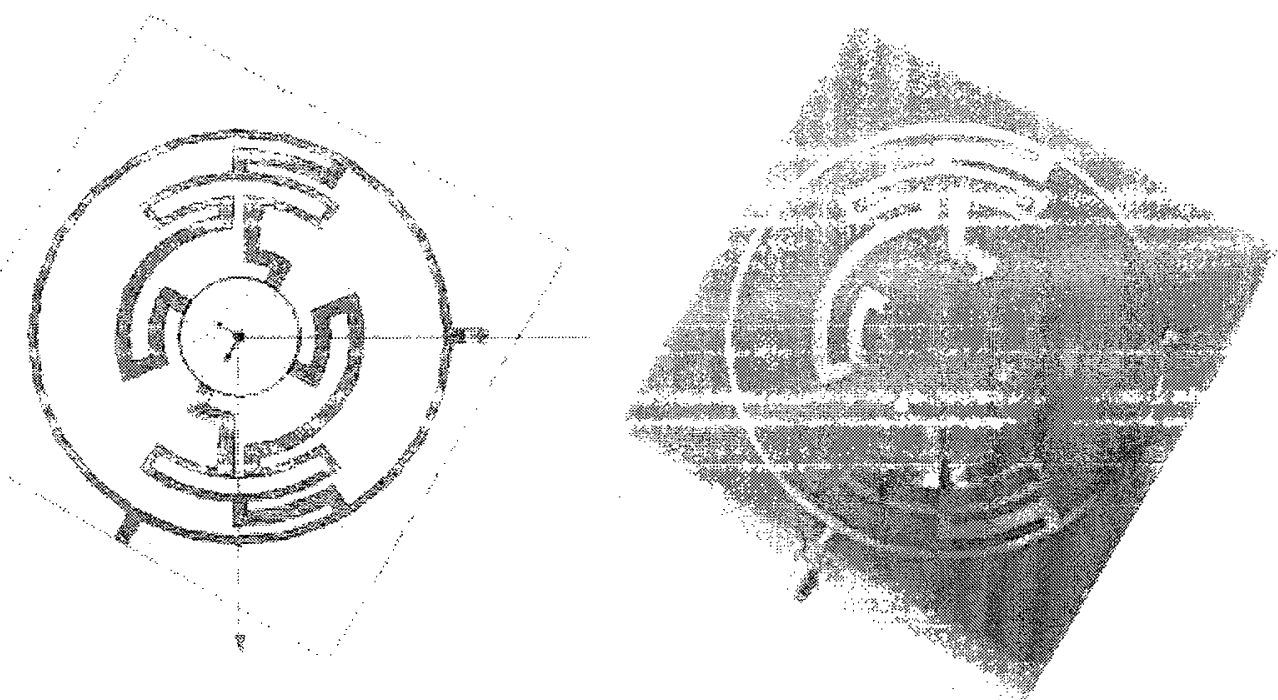

Figure 5.21: QFHA Feed network, HFSS Model (Left) PCB (Right)

The phase at each output port is determined by the angle of $\mathrm{S}_{21}$. And the measured phase at each port has been plotted in Figure 5.22. 


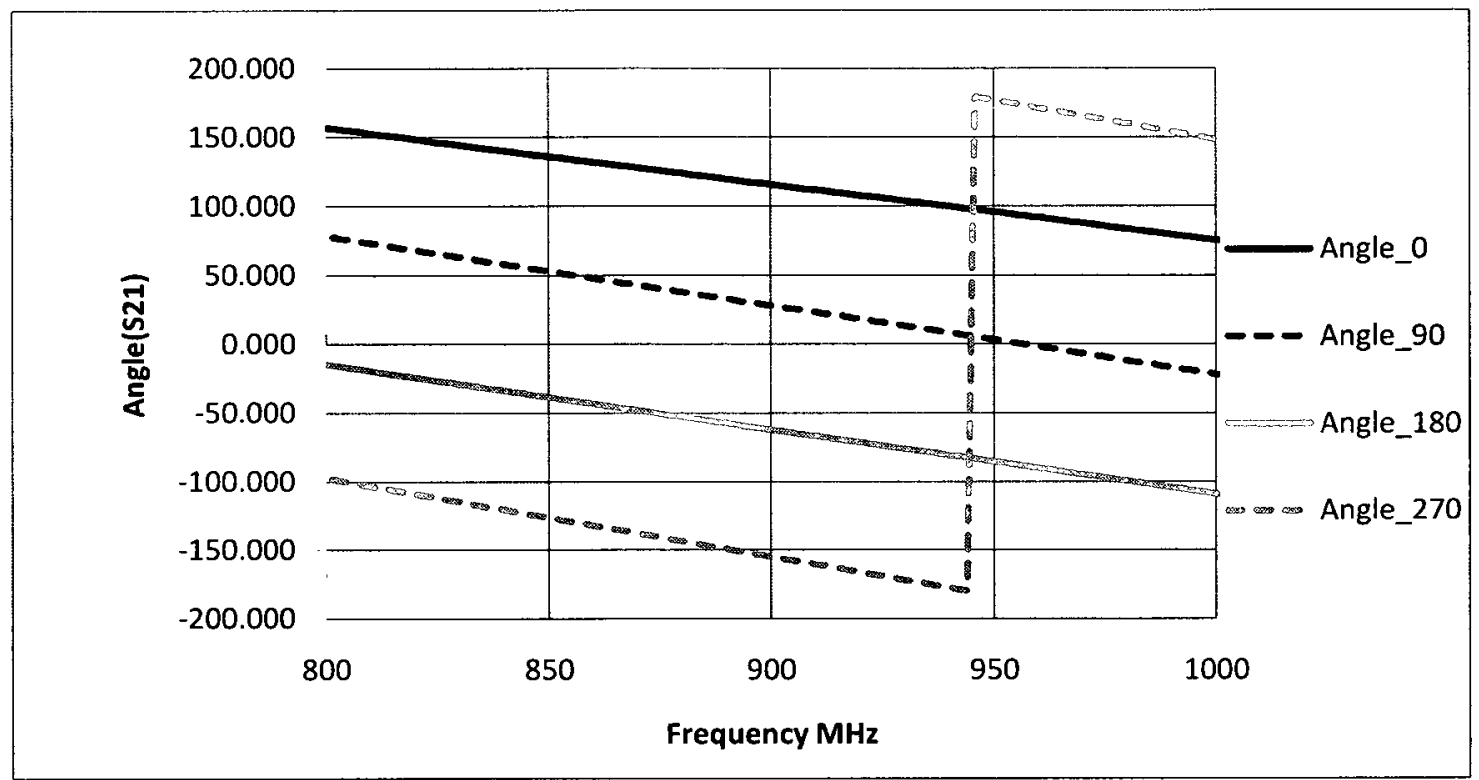

Figure 5.22: PCB Feed network Measured Phase vs. Frequency

From Figure 5.22, it can be seen that the phase difference remains constant over a large bandwidth. Since it is difficult to read the precise phase from Figure 5.22, the phase differences between the four ports measured at $915 \mathrm{MHz}$ have been tabulated in Table 5.2.

Table 5.2: Measured Phase Difference between PCB Feed network Ports at $915 \mathrm{MHz}$

\begin{tabular}{|l|l|}
\hline Phase Difference & Value \\
\hline \hline Port 1 - Port 2 & $89.0^{\circ}$ \\
\hline Port 1 - Port 3 & $178.9^{\circ}$ \\
\hline Port 1 - Port 4 & $272.8^{\circ}$ \\
\hline
\end{tabular}

The results are excellent considering the ideal phases are $90^{\circ}, 180^{\circ}$, and $270^{\circ}$. The precision on the phase is important to the overall performance of the antenna. It should be noted that over the 902-928 $\mathrm{MHz}$ range the phase difference varies by only $\pm 5^{\circ}$ from the ideal phasing. 
For optimal performance, it is important to have equal amplitude at each output port. The feed network splits the input signal in four directions so that the ideal amplitude would be $-6 \mathrm{~dB}(1 / 4$ the power) at each output port. A plot of the four amplitudes can be seen in Figure 5.23 .

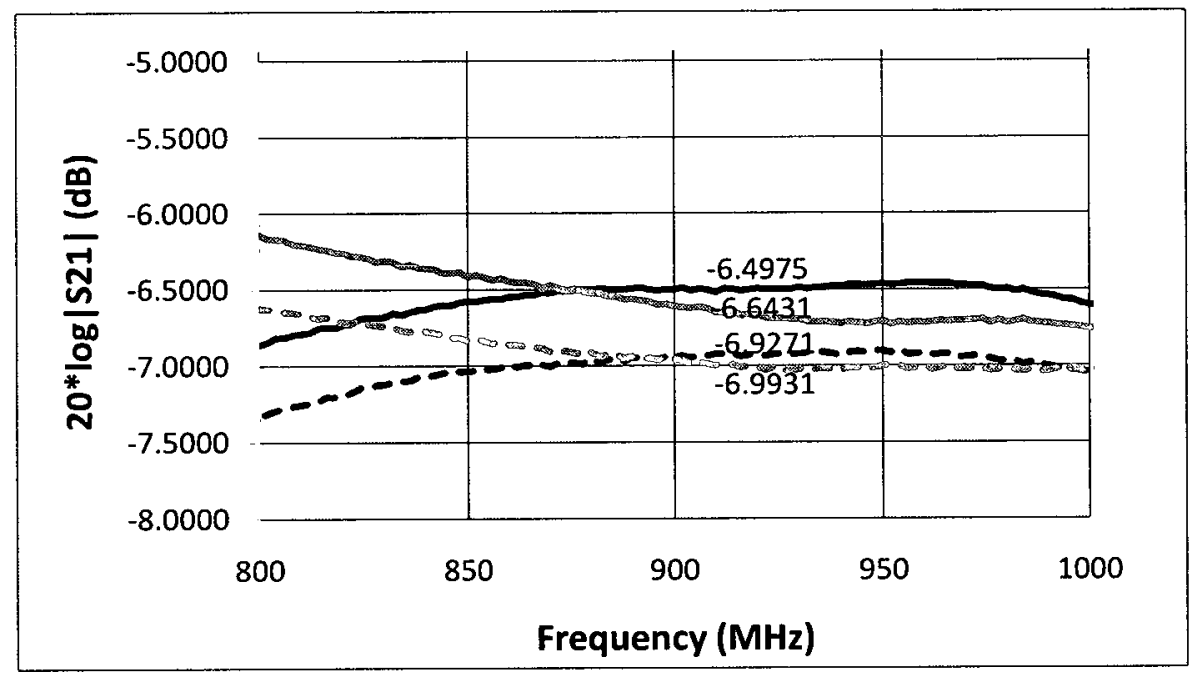

Figure 5.23: Amplitude of Signals Output from Feed network

Labels have been placed on the relative amplitudes at $915 \mathrm{MHz}$ in Figure 5.23. The loss from the feed network for the $270^{\circ}$ path is about $1 \mathrm{~dB}$, and the variation in amplitude between signals is $.5 \mathrm{~dB}$. By using a less lossy substrate material, the performance of the antenna can be improved. In the previous section it was found that each arm of the antenna has an input impedance of around $42.8+30.1 \mathrm{j} \Omega$, but the feed network has an impedance of $50 \Omega$ at each arm. This results in a mismatch loss that will reduce the overall gain of the antenna. The return loss and mismatch loss at 902,915 , and $928 \mathrm{MHz}$ are tabulated in Table 5.3. 


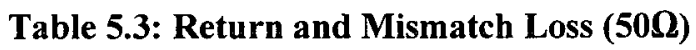

\begin{tabular}{|l|l|l|l|}
\hline Frequency & Antenna Impedance & Return Loss & Mismatch Loss \\
\hline $902 \mathrm{MHz}$ & $32.6+13.4 \mathrm{j} \Omega$ & $11.6 \mathrm{~dB}$ & $0.31 \mathrm{~dB}$ \\
\hline $915 \mathrm{MHz}$ & $42.8+30.1 \mathrm{j} \Omega$ & $10.0 \mathrm{~dB}$ & $0.46 \mathrm{~dB}$ \\
\hline $928 \mathrm{MHz}$ & $62.3+49.7 \mathrm{j} \Omega$ & $7.6 \mathrm{~dB}$ & $0.83 \mathrm{~dB}$ \\
\hline
\end{tabular}

The mismatch loss is less than $0.83 \mathrm{~dB}$ over the band of interest and the gain of the antenna will vary $0.5 \mathrm{~dB}$ over the 902 to $928 \mathrm{MHz}$ range due to this mismatch. These losses have been considered acceptable for a prototype antenna, but could be improved by incorporating a matching network.

\subsection{QFHA Fabrication and Testing}

For the final prototype, the feed network shown in Figure 5.21 was affixed to the Teflon rod and the helical conductors were soldered to the feed network. The HFSS model and prototype antenna are shown in Figure 5.24. 


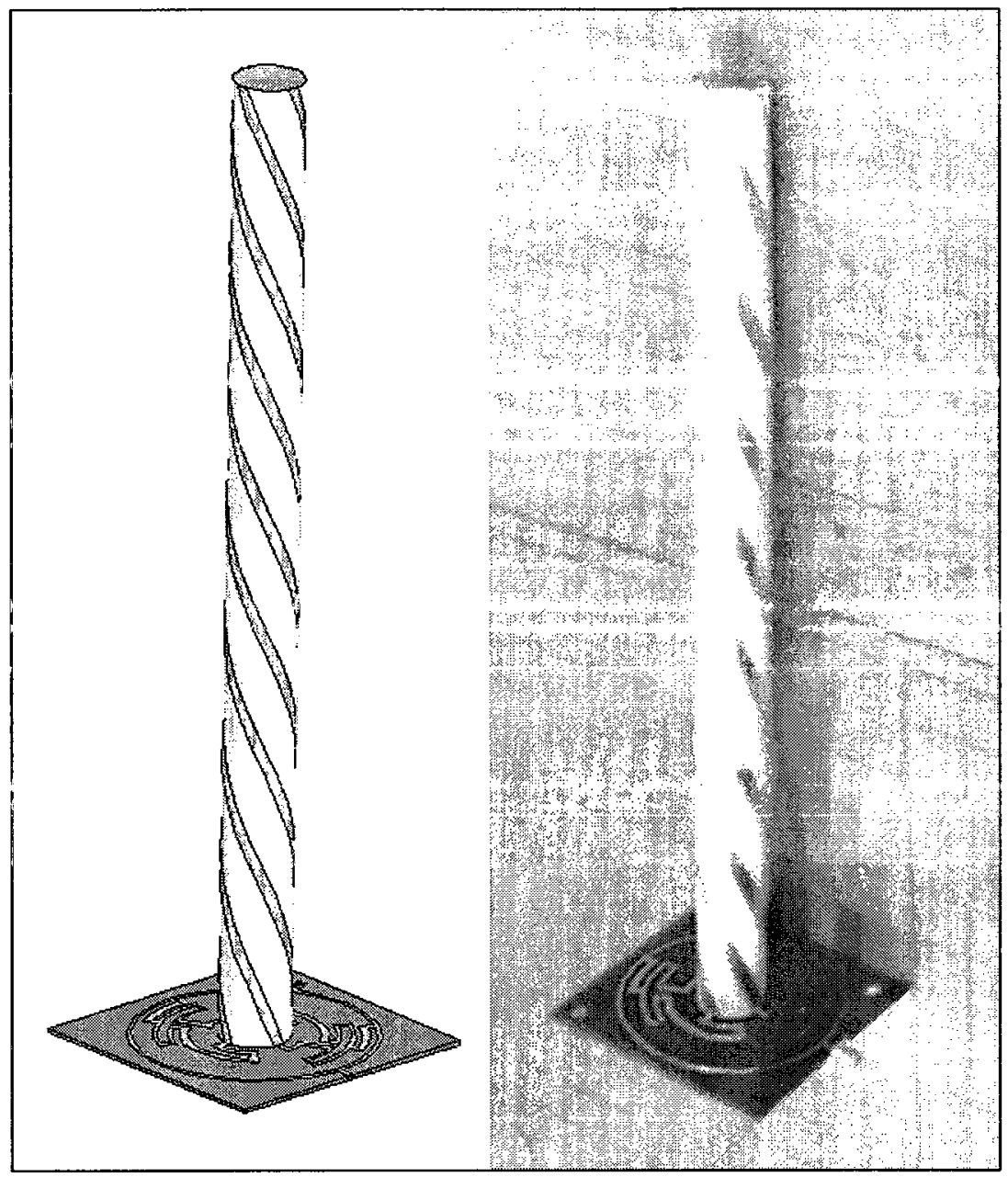

Figure 5.24: QFHA Prototype: HFSS Model (Left) Fabricated (Right)

\subsubsection{QFHA Radiation Pattern}

The prototype antenna was tested in the anechoic chamber at the Communications Research Center (CRC) in Ottawa Canada. Figure 5.25 through Figure 5.27 show comparisons between the HFSS Simulation including losses and the prototype's measured gain. 
Note: It was found through experimentation (Figure 5.20) that the fabricated prototype performs at a frequency 10 to $15 \mathrm{MHz}$ higher than the simulated antenna predicts. Therefore the comparison has been done with the simulated model operating at a frequency $13 \mathrm{MHz}$ below the fabricated antenna.

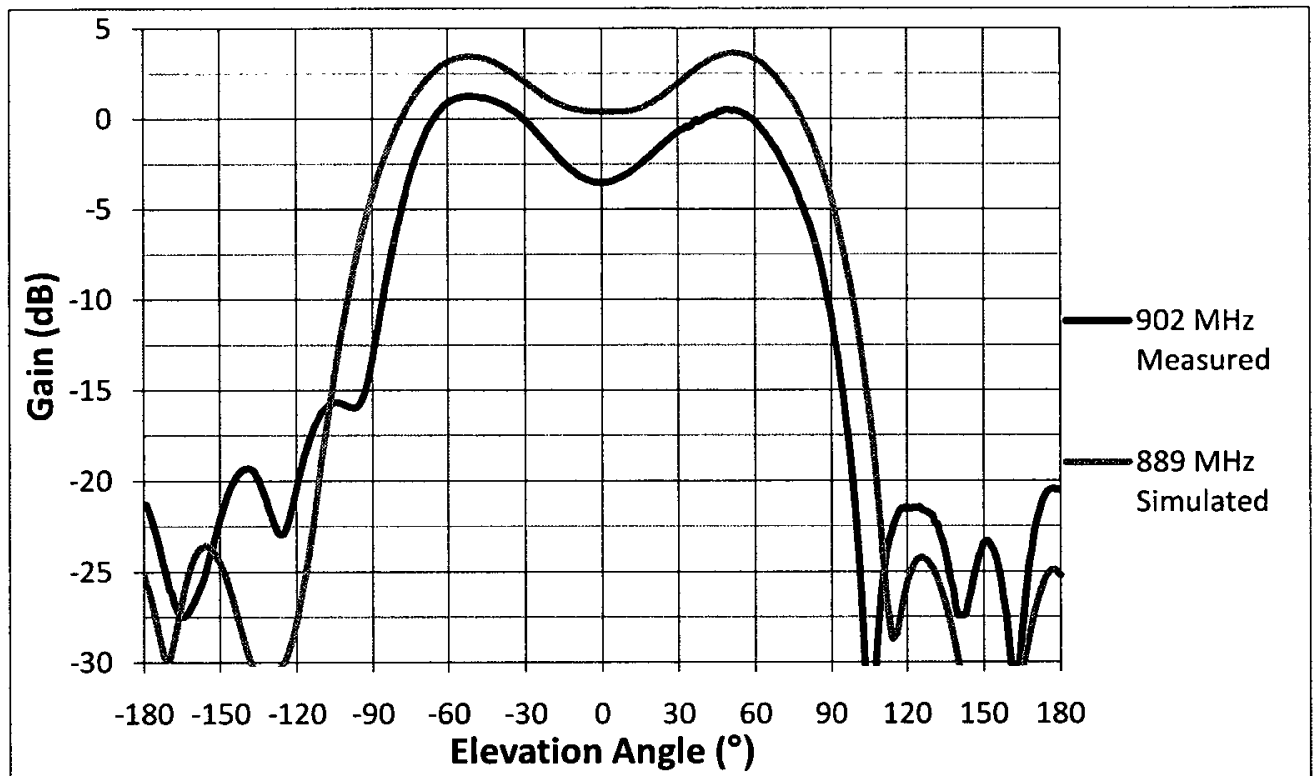

Figure 5.25: Simulated and Measured (902 MHz) Elevation Pattern of QFHA, $902 \mathrm{MHz}$ 


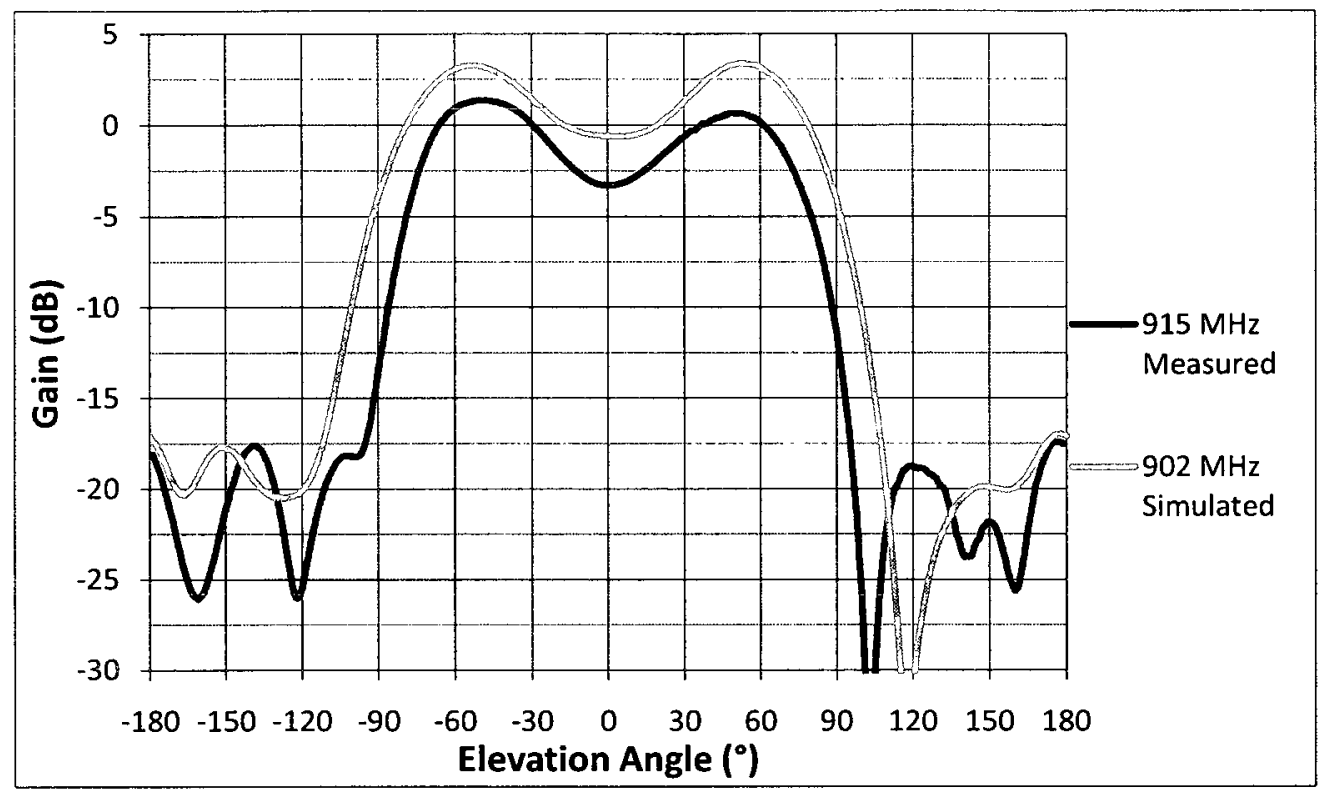

Figure 5.26: Simulated and Measured (915 MHz) Elevation Pattern of QFHA, $915 \mathrm{MHz}$

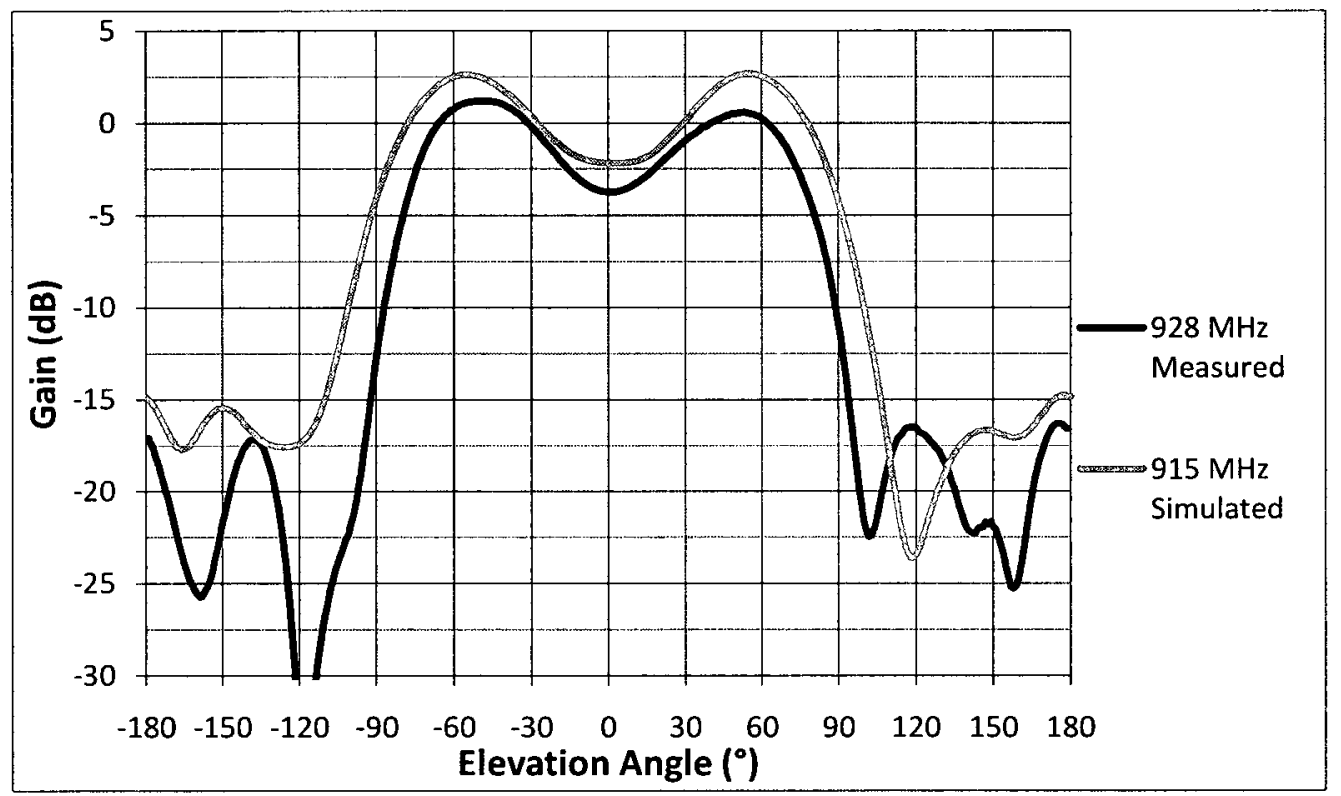

Figure 5.27: Simulated and Measured (928 MHz) Elevation Pattern of QFHA 
It is seen that the pattern shape is in close agreement with the simulation but the gain of the measured antenna is low by approximately $2 \mathrm{~dB}$. This is unexpected since the simulation accounts for all conductor, dielectric, and mismatch losses. In conclusion, there are significant losses that are unaccounted for in the simulation. The ideal lossless simulation has a peak gain of $4.75 \mathrm{dBic}$ at a $56^{\circ}$ elevation angle. The lossy QFHA model has a peak gain of $3.38 \mathrm{dBic}$ at $56^{\circ}$, while the measured prototype has peak gain of $1.35 \mathrm{dBic}$ at a $50^{\circ}$ elevation angle. Simulations show the following breakdown of loss factors:

Table 5.4: Breakdown of Losses in QFHA Simulation at $915 \mathrm{MHz}$

\begin{tabular}{|l|l|}
\hline Peak Gain- Simulated Lossless Antenna & 4.75 dBic \\
\hline Teflon Rod Dissipation & $0.13 \mathrm{~dB}$ \\
\hline Helical Strips (Copper Finite Conductivity) & $0.09 \mathrm{~dB}$ \\
\hline Mismatch Loss (Antenna and Feed network) & $0.45 \mathrm{~dB}$ \\
\hline Feed network Dissipation & $0.71 \mathrm{~dB}$ \\
\hline Peak Gain - Simulated Lossy Antenna & $\mathbf{3 . 3 8} \mathrm{dBic}$ \\
\hline Peak Gain- Measured Prototype Antenna & $1.35 \mathrm{dBic}$ \\
\hline
\end{tabular}

While the simulation accounts for many of the losses in the antenna, the fabricated antenna has an additional $2.0 \mathrm{~dB}$ of loss that is unaccounted for in the simulation. Referring to the desired radiation pattern in Figure 5.6, the gain is required to be at least $-1.5 \mathrm{~dB}$ at boresight and $3.5 \mathrm{~dB}$ at $56^{\circ}$. The simulated antenna is very close to meeting these requirements. By using a higher quality microwave material (rather than FR-4) for the feednetwork and with a matching network 
incorporated, the antenna would meet the performance requirements. However, the fabricated antenna is far from meeting the gain requirements since the losses in the antenna significantly reduce the gain of the prototype antenna. Investigation and resolution of these losses, to produce a more efficient antenna has been left as future work. The first step would be to verify the various losses experimentally especially the conductor and dielectric losses.

\subsubsection{QFHA Return Loss}

The reflection from the QFHA was found by connecting the input SMA of the feed network to a HP8720-ES network analyzer. A comparison between the simulated and fabricated antenna return loss can be seen in Figure 5.28.

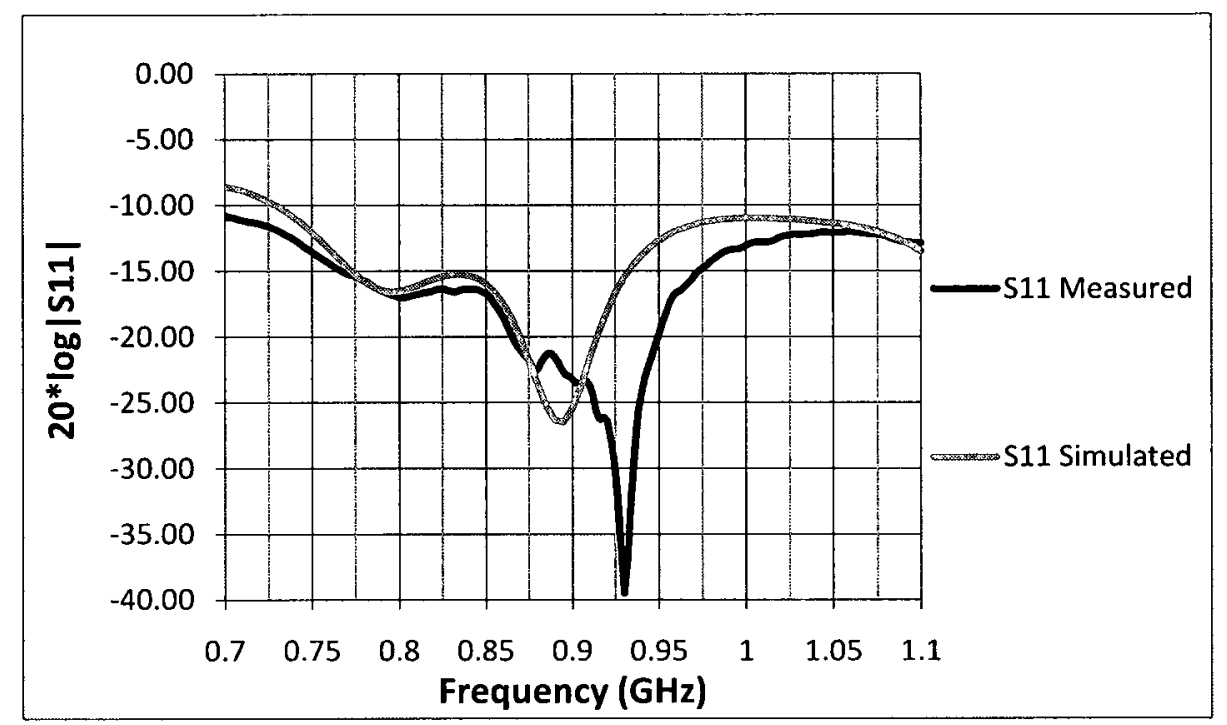

Figure 5.28: Return Loss of QFHA Simulation Prototype

From Figure 5.28 the return loss is excellent at least $20 \mathrm{~dB}$ over the band of interest. Comparing the simulated and measured results, the simulation is again predicting a lower frequency response. The return loss valley is measured at $930 \mathrm{MHz}$ while the simulated return loss valley occurs at $890 \mathrm{MHz}$. A shift of $40 \mathrm{MHz}$ can easily be accounted for with fabrication tolerances. 


\subsubsection{QFHA Phase and Gain versus Azimuth Angle}

An important parameter for the use of the antenna as an interferometer is the variation of phase and gain versus azimuth angle. The QFHA antenna was tested at the CRC where the antenna could be rotated $360^{\circ}$ in azimuth at different elevation cuts. A diagram describing the test configuration is shown Figure 5.29 for an elevation cut of $15^{\circ}$.
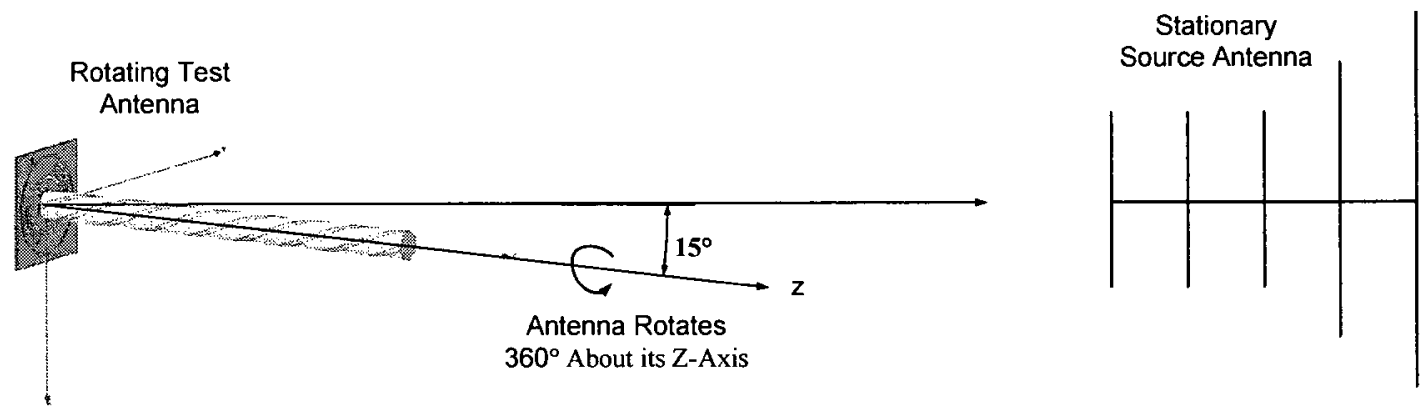

Figure 5.29: The Phase versus Angle Test Setup at a $15^{\circ}$ Elevation Cut

Theoretically, a circularly polarized antenna would have a linear phase change of $360^{\circ}$ as it is rotated a full turn azimuthally. The antenna was tested at incremental elevation cuts of $15^{\circ}$. Figure 5.30 depicts the phase change as a function of azimuth angle at a $60^{\circ}$ elevation cut. 


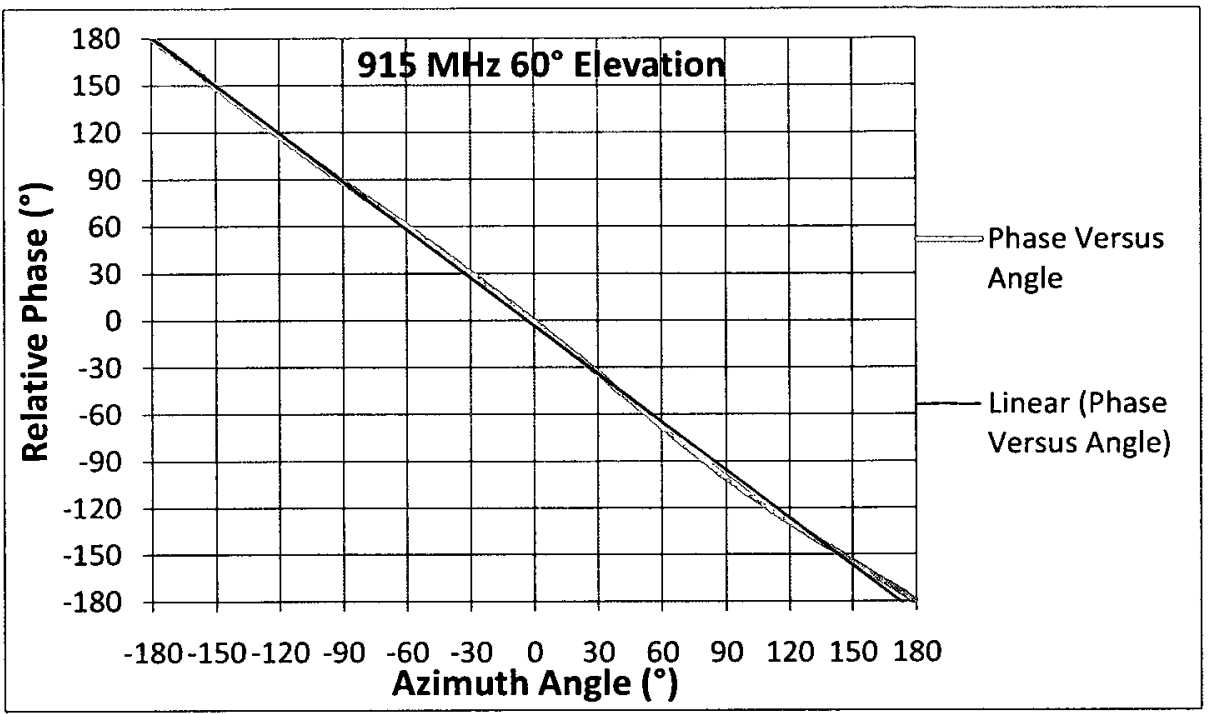

Figure 5.30: QFHA Phase Versus Azimuth Angle at 60 degree Elevation Cut

The phase changes very linearly over 360 degrees as expected, and the maximum deviation from the linear line is $5.2^{\circ}$ with a standard deviation of $2.7^{\circ}$.

An antenna with perfect circular polarization would have completely flat gain as a function of azimuth angle when tested against a linear antenna. The gain of the antenna with azimuth angle has also been plotted in Figure 5.31. 


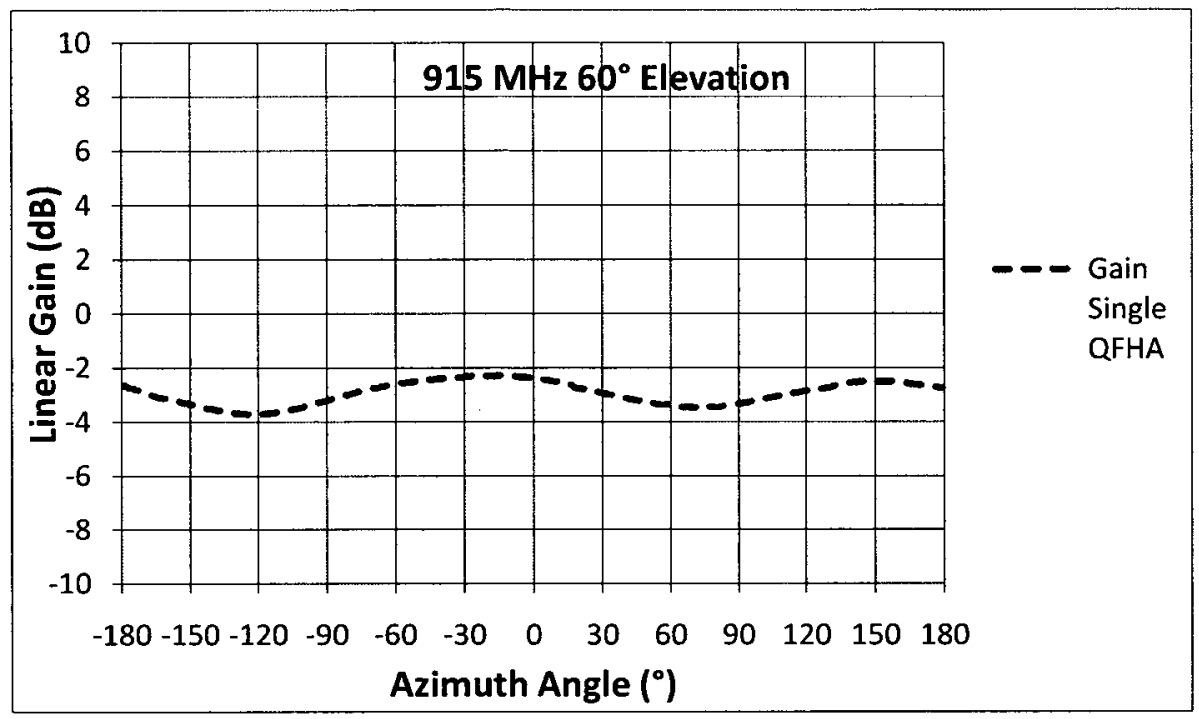

Figure 5.31: QFHA Linear Gain Versus Azimuth Angle at 60 Degree Elevation Cut

The symmetrical variation in amplitude is caused by the imperfect circular polarization. Since the antenna has been tested with a linearly polarized antenna, the difference between the maximum and the minimum gain is the axial ratio of the antenna. At the $60^{\circ}$ elevation cut, the axial ratio of the antenna is $1.4 \mathrm{~dB}$, with a standard deviation of $0.4 \mathrm{~dB}$. The results from the elevation cuts at all $15^{\circ}$ intervals are tabulated in Table 5.5. 
Table 5.5: Linear Gain and Phase Deviation of a Single QFHA

\begin{tabular}{|c|c|c|c|c|c|c|}
\hline $\begin{array}{c}\text { Elevation } \\
\text { Angle } \\
\left(^{\circ}\right)\end{array}$ & $\begin{array}{l}\text { Average } \\
\text { Gain } \\
\text { (dB) }\end{array}$ & $\begin{array}{c}\text { Maximum } \\
\text { Gain } \\
\text { (dB }\end{array}$ & $\begin{array}{l}\text { Minimum } \\
\text { Gain } \\
\text { (dB) }\end{array}$ & $\begin{array}{c}\text { Gain } \sigma \\
\text { (dB) }\end{array}$ & $\begin{array}{c}\text { Max Phase } \\
\text { Variance } \\
\left(^{\circ}\right)\end{array}$ & $\begin{array}{c}\text { Phase } \sigma \\
\left(^{\circ}\right)\end{array}$ \\
\hline $0^{\circ}$ & -7.4 & $\begin{array}{l}-6.3 \\
\end{array}$ & -8.8 & 0.8 & 7.8 & 5.3 \\
\hline $15^{\circ}$ & -6.5 & -5.6 & -7.7 & 0.7 & 7.4 & 4.2 \\
\hline $30^{\circ}$ & -4.4 & -3.4 & -5.5 & 0.7 & 6.8 & 3.9 \\
\hline $45^{\circ}$ & -2.8 & -2.2 & -3.7 & 0.5 & 5.5 & 3.1 \\
\hline $60^{\circ}$ & -3 & -2.3 & -3.7 & 0.4 & 5.2 & 2.7 \\
\hline $75^{\circ}$ & -5.8 & -4.7 & -6.7 & 0.6 & 6.5 & 4.2 \\
\hline $90^{\circ}$ & -13.1 & -15.2 & -11.5 & 1.1 & 14.5 & 7.7 \\
\hline
\end{tabular}

Table 5.5 provides data on the deviation in gain and phase of the antenna. By taking the difference in minimum and maximum gain, the axial ratio of the antenna has been found to be better than $2.5 \mathrm{~dB}$ over an elevation angle of $0^{\circ}-75^{\circ}$. The phase variation over the $0^{\circ}-75^{\circ}$ range is also very low with less than $\pm 7.8^{\circ}$ maximum deviation.

\subsection{QFHA Interferometer Array}

Three QFHA antennas have been configured in an equilateral triangle with half wavelength spacing as shown in Figure 5.32. The array is to be used as an interferometer for direction finding as described in Chapter 4. It is interesting to see the effect of the array configuration on the performance of the individual antennas; what is particularly interesting is the phase and amplitude distortion resulting from these three dimensional structures. The QFHA interferometer array can be seen in Figure 5.32. 


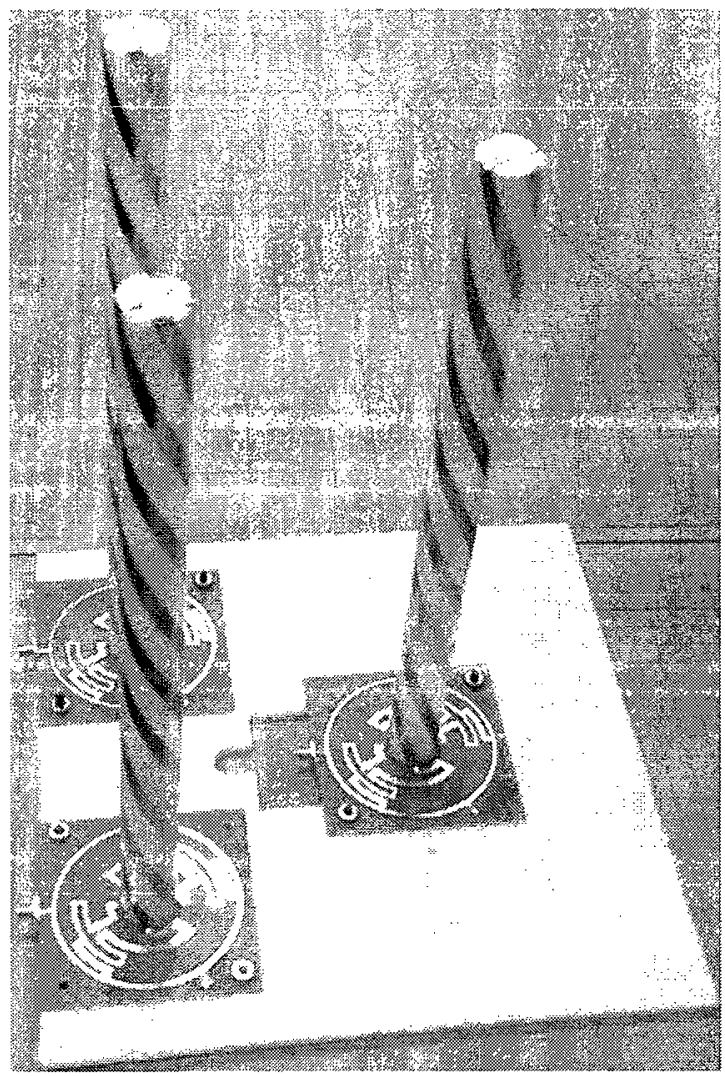

Figure 5.32: Fabricated QFHA Interferometer Array

\subsubsection{Mutual Coupling}

Helical antennas have low mutual coupling and are often used in arrays [13]. The mutual coupling between the QFHA elements was determined by connecting two of the antenna ports to a VNA and the third antenna to a $50 \Omega$ termination. The $S_{21}$ measurement is plotted in Figure 5.33 . 


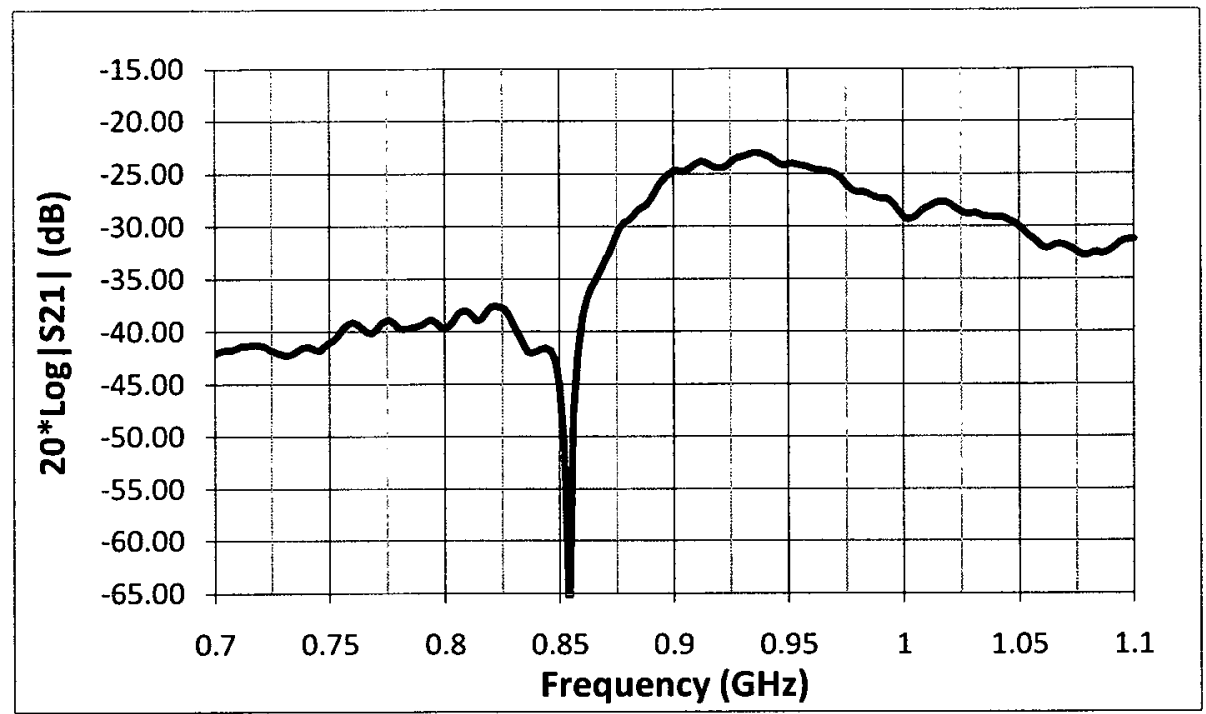

Figure 5.33: Measured Mutual Coupling between Two QFH Antennas in the Interferometer Configuration

The mutual coupling between the two antennas is better than $-23 \mathrm{~dB}$ over the 902 to $928 \mathrm{MHz}$ frequency range.

\subsubsection{QFHA Array Phase and Gain versus Azimuth Angle}

The antenna array has been measured at the Communication Research Center (CRC) to test the phase and gain versus angle. One antenna was measured individually in the array configuration, while the remaining two antennas were terminated with a $50 \Omega$ load. The antenna was rotated about its own axis as depicted in Figure 5.34. A photo of the QFHA array mounted in the CRC Anechoic Chamber can be seen in Figure 5.35. 



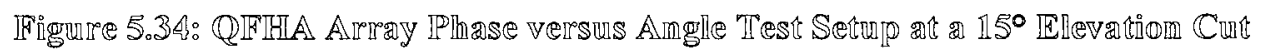

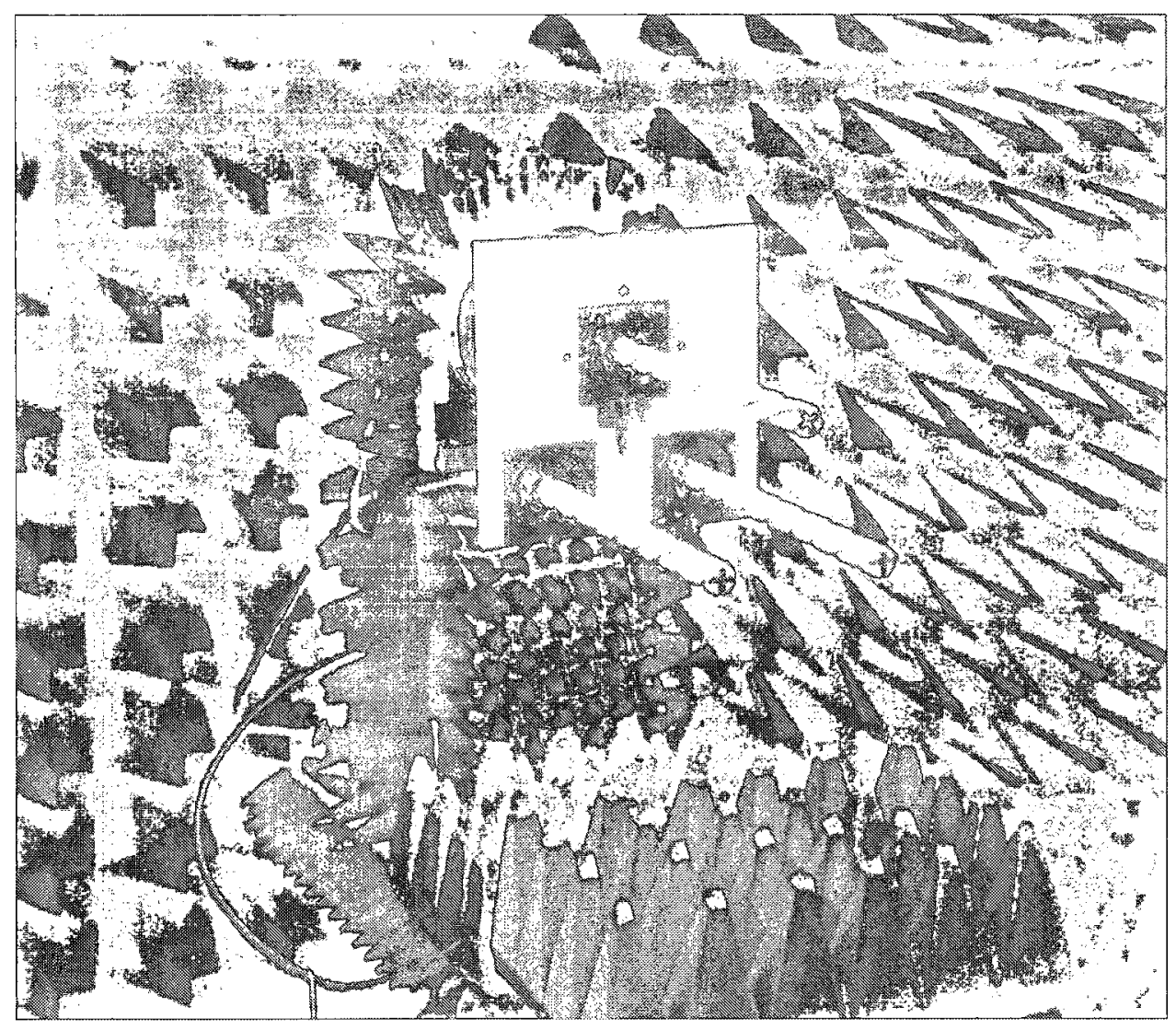

Figune 5.35: QFHA Array Mounted im the Amechoic Chamber at the Communicatiom Resemren Center 
The purpose of this test was to compare the amplitude and phase distortion of the antenna in an array configuration with the single antenna on its own. The additional antenna elements create additional amplitude and phase distortion when compared with the results from the single element test. The phase and magnitude variance can be seen in Figure 5.36 and Figure 5.37 at a $60^{\circ}$ elevation cut.

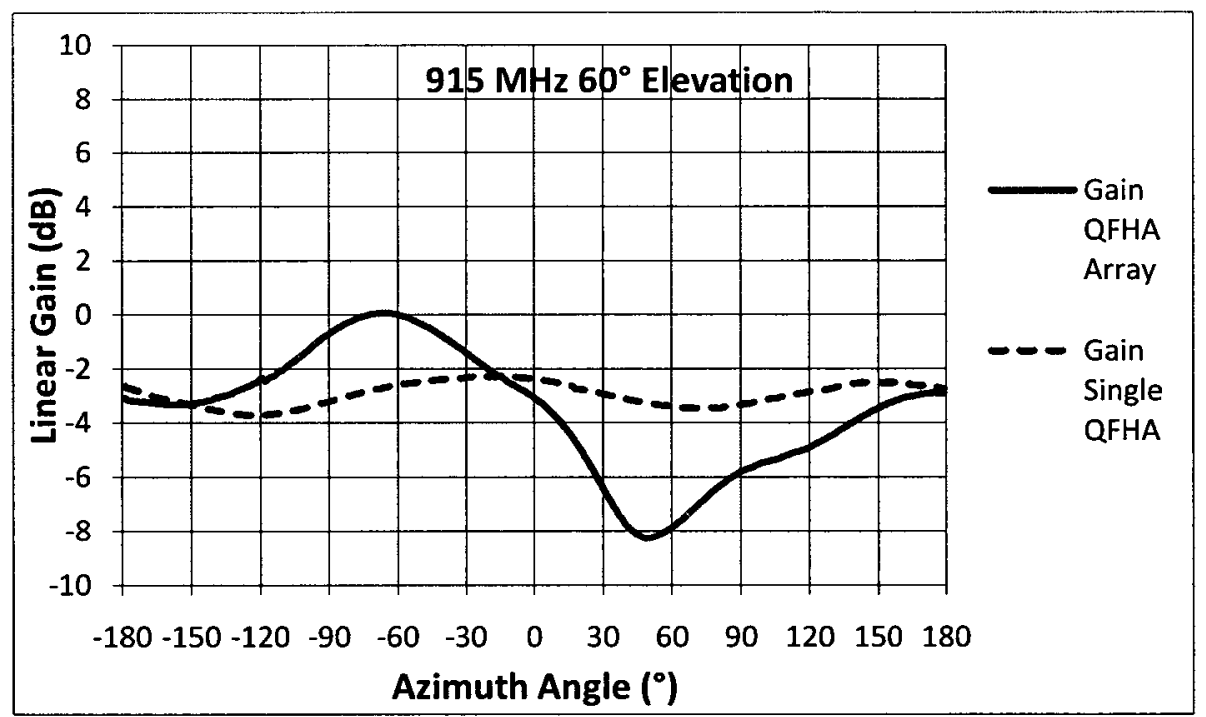

Figure 5.36: Gain Comparison of QFHA and the QFHA in an Array at a $60^{\circ}$ Elevation 


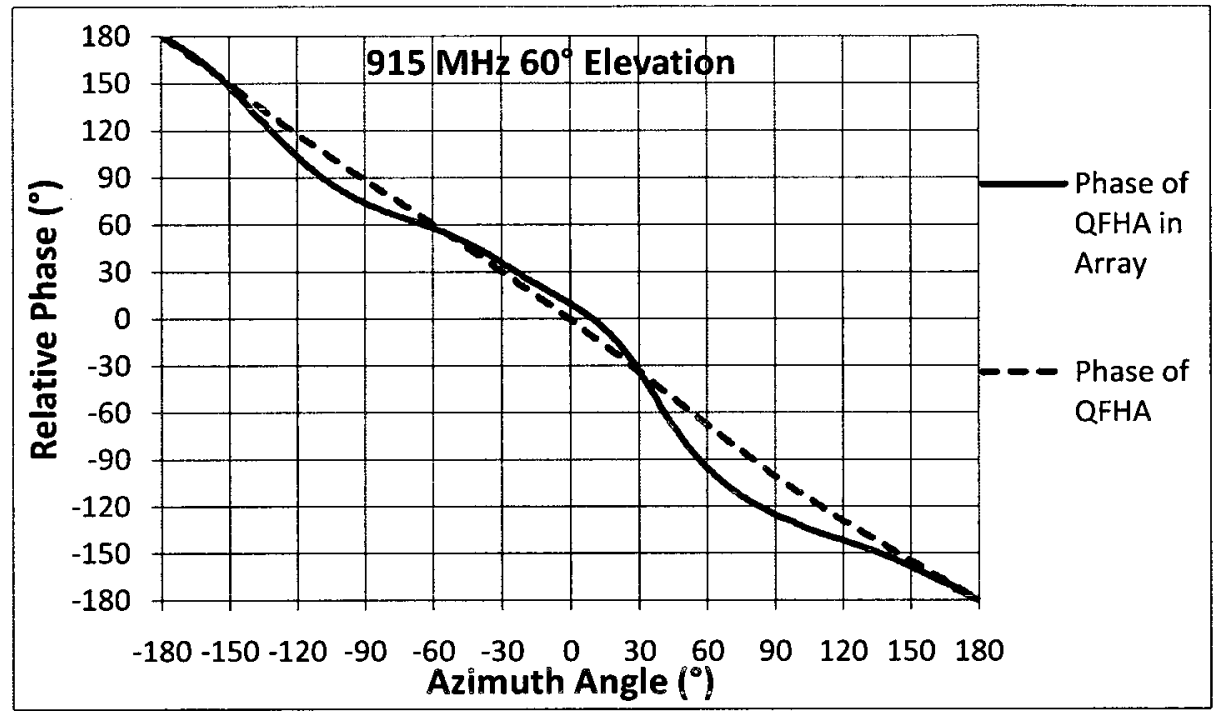

Figure 5.37: Phase Comparison of QFHA and the QFHA in an Array at a $60^{\circ}$ Elevation From Figure 5.36 and Figure 5.37 it is clear that the presence of the additional antennas distorts the signal. The results from the phase and gain measurements of the QFHA array at $15^{\circ}$ elevation intervals have been tabulated below:

Table 5.6: Gain and Phase Deviation of QFHA Array

\begin{tabular}{|c|c|c|c|c|c|c|}
\hline $\begin{array}{c}\text { Elevation } \\
\text { Angle } \\
\left({ }^{\circ}\right)\end{array}$ & $\begin{array}{c}\text { Average } \\
\text { Gain } \\
\text { (dB) }\end{array}$ & $\begin{array}{c}\text { Maximum } \\
\text { Gain } \\
\text { (dB) }\end{array}$ & $\begin{array}{l}\text { Minimum } \\
\text { Gain } \\
\text { (dB) }\end{array}$ & $\begin{array}{r}\text { Gain } \sigma \\
\text { (dB) }\end{array}$ & $\begin{array}{c}\text { Max Phase } \\
\text { Variance } \\
\left(^{\circ}\right)\end{array}$ & $\begin{array}{c}\text { Phase } \sigma \\
\left(^{\circ}\right)\end{array}$ \\
\hline$\overline{0^{\circ}}$ & -3.0 & -1.32 & $\overline{-4.6}$ & 0.9 & 8.5 & 5.5 \\
\hline $15^{\circ}$ & -2.6 & -0.8 & -5.2 & 1.3 & 14.0 & 8.2 \\
\hline $30^{\circ}$ & 0.5 & 3.3 & -4.1 & 2.2 & 13.7 & 9.3 \\
\hline $45^{\circ}$ & -.9 & 2.7 & 6.7 & 2.8 & 19.4 & 11.3 \\
\hline $60^{\circ}$ & -.54 & 3.1 & -5.3 & 2.3 & 24.3 & 13.3 \\
\hline $75^{\circ}$ & -2.4 & .8 & -7.7 & 2.1 & 28.0 & 16.7 \\
\hline $90^{\circ}$ & -8.1 & -3.9 & -15.7 & 3.0 & 45.1 & 14.8 \\
\hline
\end{tabular}


Table 5.6 summarizes the results from the array measurements. Comparing the results with Table 5.5 , the variation is similar at the $0^{\circ}$ elevation angle. When the elevation angle increases, the other antennas produce significant distortion of the signal. At a $60^{\circ}$ elevation cut, the single antenna has a standard deviation in gain and phase of $.4 \mathrm{~dB}$ and $2.7^{\circ}$ respectively. However, the array test shows that at the $60^{\circ}$ elevation cut there is a gain and phase standard deviation of 2.3 $\mathrm{dB}$ and $13.3^{\circ}$. This additional distortion at higher elevation angles is not surprising since the QFHA is a 3-D structure which physically obstructs the signal in an array configuration. The phase and amplitude distortion are not linear and calibration will be necessary to use these antennas in an interferometer array for direction finding.

\subsection{QFHA Summary}

The QFHA antenna has been successfully designed and fabricated. The fabricated antenna has the radiation pattern shown in Figure 5.38 . 


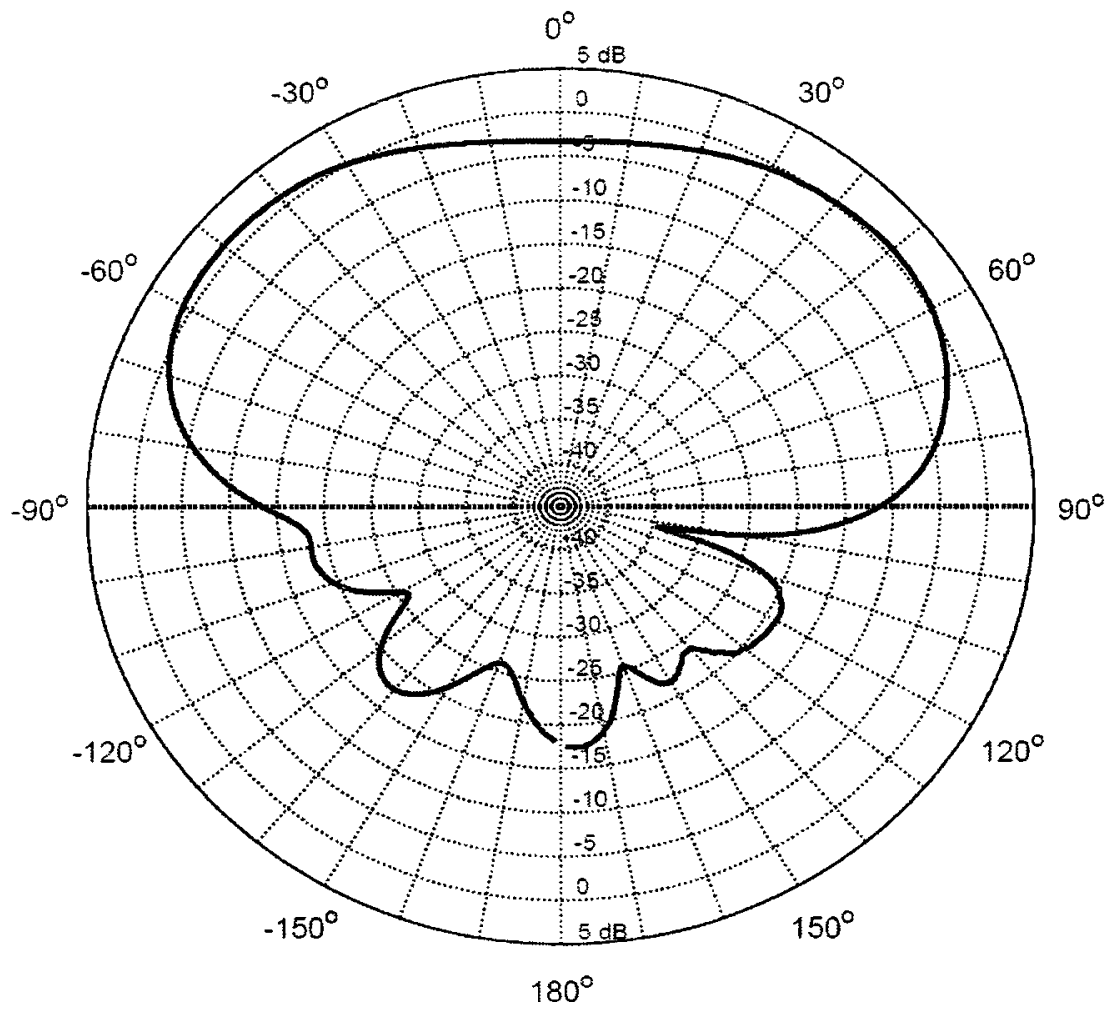

Figure 5.38: QFH RFID Reader Antenna Elevation Cut in dBic (Measured at 915 MHz)

While the pattern shape of the antenna is correct, the major issue is that the measured gain is $2 \mathrm{~dB}$ lower than expected. The antenna is required to have a peak gain of at least $3.5 \mathrm{dBic}$ at a $56^{\circ}$ elevation cut. Simulations show that the antenna is capable of meeting this requirement, and improving the efficiency of the prototype antenna is left for future work. Except for the reduced gain, the antenna performs very well as a UHF RFID antenna. The specifications of the antenna are summarized in Table 5.7. 
Table 5.7: Performance Characteristics of QFHA for UHF RFID Reader

\begin{tabular}{|l||l||}
\hline Frequency of Operation & $902-928 \mathrm{MHz}$ \\
\hline Peak Gain & $1.35 \mathrm{dBic}\left(50^{\circ}\right.$ Elevation angle) \\
\hline Polarization & LHCP \\
\hline Axial Ratio (Maximum) $\left(30^{\circ}-75^{\circ}\right)$ & $2.1 \mathrm{~dB}$ \\
\hline 3 dB Beamwidth & $70^{\circ}-20^{\circ}$ \\
\hline Front to Back Ratio & $-14.8 \mathrm{~dB}$ \\
\hline \hline Return Loss Minimum $(50 \Omega$ reference) & $20 \mathrm{~dB}$ \\
\hline Ground Plane Size & $10 \mathrm{~cm}^{2}$ \\
\hline \hline Teflon Rod Diameter & $1.27 \mathrm{~cm} \mathrm{(1")}$ \\
\hline \hline Antenna Height & $36 \mathrm{~cm}$ \\
\hline
\end{tabular}

It was expected that since the QFHA is a 3-D structure, the antenna would have significant phase and amplitude distortion when configured in the interferometer array. For example, at $60^{\circ}$ elevation angle, the results show that the single QFHA has a phase versus azimuth angle standard deviation of $2.7^{\circ}$; however, when the antenna is placed in the interferometer, the standard deviation increases to $13.3^{\circ}$. From the DOA error analysis in Table 3.1 , this corresponds to a DOA estimation with a standard deviation around $4^{\circ}$. Calibration of the interferometer array is required to improve the accuracy of the system. 


\section{Chapter 6}

\section{Conclusions and Future Work}

\subsection{Summary and Contributions}

The purpose and objective of the study was to investigate the design of an interferometer array for use with UHF RFID tags. It was necessary to design a custom antenna due to the unique size, polarization, and gain requirements of this application. A three element interferometer in an equilateral triangle configuration was selected. The configuration uses elements spaced a half wavelength apart and is capable of providing unambiguous direction estimation in a $360^{\circ}$ field of view. Monte Carlo simulations were performed to understand how phase error at the antenna effects the DOA estimation. The results are tabulated in Table 3.1 which shows that the interferometer is fairly robust to phase deviation.

Two interferometer arrays were designed and constructed. The first was built using microstrip patch antenna elements while the second uses a nontraditional QFHA. The microstrip patch antenna was custom designed to have circular polarization and size reduced so that it would fit in the chosen array configuration. The patch antenna proved to be low cost, and met the requirements of a North American UHF RFID antenna.

A QFHA was also investigated as a viable candidate for UHF RFID. The major benefit of this antenna is its ability to produce a shaped radiation pattern. Considering any real world situation where the antenna is centered above a coverage area, it is beneficial to have peak gain at an elevation angle offset from boresight. A shaped beam QFHA will outperform the traditional patch antenna when used in this situation. While simulation and measured results of the QFHA agree quite well, the additional loss in the fabricated prototype was not expected and significantly 
degrades the radiation efficiency of the antenna. The losses that occur in the prototype require further investigation; however, it is believed that the losses can be controlled and the efficiency can be improved by at least $2 \mathrm{~dB}$, making the antenna a possible candidate for a commercial RFID application.

There are three major contributions that come from this work. First, it has been proven that a low cost and practical interferometer array can be fabricated for use in a commercial UHF RFID application. Second, the design procedure of the QFHA used graphical techniques combined with modern simulation tools as a starting point, and is a significant contribution. Compared to the early 1970's when Kilgus experimentally plotted curves for the QFHA, this procedure was effective and will be beneficial to other designers. Finally, it has been shown that at low elevation angles the patch antenna array and the quadrifilar have significant phase distortion. Even though the patch antenna is a two dimensional structure the phase distortion was still considerable. For example, at a $60^{\circ}$ elevation angle, the phase deviation versus azimuth angle had a standard deviation of $7.2^{\circ}$ for the patch antenna, while the quadrifilar array has a standard deviation of $13.3^{\circ}$. The results of the Monte Carlo simulation in Table 3.1 show that if the phase deviates with a standard deviation of $10^{\circ}$, this produces a DOA estimation error with a standard deviation of $3.6^{\circ}$. Without calibration this is approximately the level of accuracy that can be expected from the interferometer array.

\subsection{Future Work}

Prototype antenna elements and arrays have been designed and built. The future work is to produce professional antennas with photo etching techniques and precise repeatable production. The unaccounted losses in the QFHA need investigation and resolution before the antenna can be used commercially. There is room to improve the performance of both antennas by using a feed 
network with a low loss microwave laminate. To improve the DOA accuracy of the interferometer array a calibration procedure should be developed.

\subsection{Conclusion}

The interferometer arrays investigated are feasible for use with low cost RFID tags. The patch antenna proved to be less expensive and easier to fabricate than the QFHA. Also, the phase versus azimuth angle of the patch interferometer array is more stable than the QFHA array. The advantage of the QFHA is that it is capable of producing a shaped radiation pattern so that peak gain is focused at elevation angles with greater path loss. This shaped pattern has the possibility to improve read distance when the antenna is centered above a coverage area. It proved difficult to fabricate a QFHA with good radiation efficiency. For an inexpensive, simple, and low profile solution the microstrip patch antenna is the best option. 


\section{Bibliography}

[1] D. Dobkin, The RF in RFID. Burlington, MA: Newnes, 2008.

[2] Y. Zhang, M. Amin, and S. Kaushik, "Localization and Tracking of Passive RFID tags based on Direction Estimation," International Journal of Antennas and Propagation, vol. 2007, p. 9, Dec. 2007.

[3] G. McKerricher and J. Wight, "Design of a Shaped Beam RFID Reader Antenna," in Antenna Technology and Applied Electromagnetics Conference, Ottawa, Jul. 2010.

[4] G. McKerricher and J. Wight, "Quadrifilar Helix Antenna for UHF RFID," in Antennas and Propagation Symposium, Toronto, Jul. 2010.

[5] Patrick J. Sweeney, RFID for Dummies. Indianapolis, Indiana: Wiley Publishing Inc., 2005.

[6] M. Roberti, "A 5-Cent Breakthrough," RFID Journal, May 2006.

[7] EPC Global Inc, "Class-1 Generation-2 UHF RFID Protocol for Communications at 860 $\mathrm{MHz}-960 \mathrm{MHz}$ Version 1.0.9," Radio Frequency Identity Protocol, 2005.

[8] L3 Communications. (2002, Jul.) L3 Communications Website. [Online]. http://www.l3com.com/randtron/infrmtech.htm

[9] Y. Zhang, X. Li, and M. G. Amin, "Principles and techniques of RFID positioning," in RFID Systems, Research Trends and Challenges. Wiley, in press (2010), ch. 18.

[10] R. Edwards, An Airborne direction finder for GPS interference sources. Ottawa: Thesis M. Eng. Carleton University, 1999.

[11] C. Balanis, Antenna Theory - Analysis and Design, 3rd ed. Hoboken, New Jersey: John Wiley \& Sons, 2005.

[12] R. Bancroft, Microstrip and Printed Antenna Design, 1st ed. Noble Publishing Corporation, 2004

[13] J. Kraus, Antennas - For all Applications, 3rd ed. Singapore: McGraw Hill Education, 2001.

[14] D. Pozar, Microwave Engineering, 3rd ed., Z. Bill, Ed. Hoboken NJ, United States of America: John Wiley \& Sons, 2005.

[15] C. Kilgus, "Resonant Quadrifilar Helix," IEEE Trans. Ants. Prop., no. AP-17, pp. 349-351, May 1969 
[16] Surrey Satellite Technology. (2010, May) S-Band Quadrifilar Helix Antenna Brochure. www.sstl.co.uk. [Online]. www.sstl.co.uk

[17] C. Kilgus, "Shaped Conical Radiation Pattern Performance of the Backfire Quadrifilar Helix," IEEE Transactions of Antennas and Propagation, pp. 392-397, May 1975.

[18] A. Kumar, Fixed and Mobile Terminal Antennas. Boston, USA: Artech House Inc., 1991.

[19] O. Pekoonen, S. Vili, K. Kiesi, M. Ermutlu, and K. Wilska, "Measuring the Input Impedance of a Quadrifilar Helix Antenna," Microwave and Optical Technology Letters, vol. 17, pp. 102-107, Feb. 1998.

[20] J. Izandian, "QFHA Antennas for Satellite and Mobile Phone Applications," Microwave Journal, vol. 45, no. 7, pp. 64-71, Jul. 2002. 